\title{
Grau de aplicações G-equivariantes entre variedades generalizadas
}


SERVIÇO DE PÓS-GRADUAÇÃO DO ICMC-USP

Data de Depósito:

Assinatura:

\title{
Grau de aplicações G-equivariantes entre variedades generalizadas
}

\author{
Norbil Leodan Córdova Neyra
}

Orientadora: Profa. Dra. Denise de Mattos

Dissertação apresentada ao Instituto de Ciências Matemáticas e de Computação - ICMC-USP, como parte dos requisitos para obtenção do título de Mestre em Ciências - Matemática . VERSÃO REVISADA

USP - São Carlos

Junho de 2014 
Ficha catalográfica elaborada pela Biblioteca Prof. Achille Bassi e Seção Técnica de Informática, ICMC/USP, com os dados fornecidos pelo(a) autor(a)

\begin{tabular}{|c|c|}
\hline \multirow[t]{3}{*}{ C796g } & $\begin{array}{l}\text { Córdova Neyra, Norbil Leodan } \\
\quad \text { Grau de aplicações G-equivariantes entre } \\
\text { variedades generalizadas / Norbil Leodan Córdova } \\
\text { Neyra; orientador Denise de Mattos. -- São Carlos, } \\
2014 . \\
\quad 78 \text { p. }\end{array}$ \\
\hline & $\begin{array}{l}\text { Tese (Doutorado - Programa de Pós-Graduação em } \\
\text { Matemática)-- Instituto de Ciências Matemáticas e } \\
\text { de Computação, Universidade de São Paulo, } 2014 \text {. }\end{array}$ \\
\hline & $\begin{array}{l}\text { 1. Teoria de grau. 2. Aplicações equivariantes. } \\
\text { 3. Variedades generalizadas. 4. Homologia de Borel- } \\
\text { Moore. 5. Cohomologia de feixes. I. de Mattos, } \\
\text { Denise, orient. II. Título. }\end{array}$ \\
\hline
\end{tabular}


A mis queridos padres Eduardo Cordova y Norma Neyra, con mucho amor. 


\section{Agradecimentos}

É com muita satisfação que expresso aqui o mais profundo agradecimento a todas as pessoas que, ao longo do meu doutorado me ajudaram, direta ou indiretamente, a cumprir os meus objetivos e a realizar mais esta etapa da minha formação acadêmica.

Inicialmente, gostaria de agradecer a Deus. Ele esteve sempre ao meu lado durante esta caminhada dando-me forças para não desistir.

A mi família (Eduardo, Norma, Javier, Milton, Nelson y Rosyzela) que incluso estando lejos, siempre estaban conmigo, enseñándome, apoyandome, amandome incondicionalmente y creendo en mi potencial.

Agradeço também a uma pessoa muito especial que faz parte da minha vida, minha noiva Patricia, por sempre estar comigo e ter me ajudado em todos os sentidos para que este trabalho fosse realizado. Amo-te muito.

É com muita admiração e respeito que agradeço à minha orientadora Dra. Denise de Mattos e ao professor Dr. Edivaldo Lopes Dos Santos por terem acreditado em mim e pelo tempo e ajuda oferecida no decorrer deste período. Os ensinamentos, dedicação paciência e seriedade profissional deles foram os fatores que conduziram este trabalho. Obrigado por aceitar-me como orientando. Nada disso teria acontecido sem vocês, muito obrigado!

Agradeço à FAPESP pelo apoio financeiro a este trabalho.

Aos meus professores e ao meus colegas do ICMC-USP e da FACFyM-UNPRG que fizeram parte da minha vida acadêmica. 
À Ana Paula, Laura e Diego Talarico pela atenção que me deram durante todo este período.

A mis amistades de Perú, por el aliento y la confianza. Em especial a mis grandes amigas de toda la vida: Angelica, Paola y Fiorela.

A todos os meus amigos em Brasil (uma lista muito longa), pela amizade, pelo auxílio e por todos os momentos inesquecíveis que passamos. Em especial, à família Pizarro Laquise pela grande amizade.

Enfim, a todos que colaboraram de alguma forma com este trabalho.

Muito obrigado!! 


\section{Resumo}

Neste trabalho estenderemos os resultados obtidos por Hara [34] e J. Jaworowski [38] substituindo as $G$-variedades por $G$-variedades generalizadas sobre $\mathbb{Z}$. Além disso, provamos uma fórmula de comparação geral para grau de aplicações de uma variedade generalizada sobre uma esfera que são equivariantes com respeito a ações de grupos finitos, obtendo uma generalização do resultado de A. Kushkuley e Z. Balanov [40]. 


\section{Abstract}

In this work, we extend the results obtained by Y. Hara [34] and J. Jaworowski [38] by replacing the free $G$-manifolds by free generalized $G$-manifolds over $\mathbb{Z}$. Moreover, we prove a general comparison formula for degrees of equivariant maps from a generalized manifold to a sphere which are equivariant with respect to finite group actions, obtaining a generalization of the result of A. Kushkuley and Z. Balanov [40]. 


\section{Sumário}

Introdução 1

CAPÍtUlo 1. Preliminares $\quad 7$

1.1. Dimensão cohomológica . . . . . . . . . . . . . . . 9 9

1.2. Feixe de homologia . . . . . . . . . . . . . . . . . 11

1.3. Conexidade local em cohomologia . . . . . . . . . . . . . . 12

1.4. Variedades generalizadas . . . . . . . . . . . . . . . . . . . 14

1.5. Módulos de cohomologia de variedades generalizadas . . . . . . . 16

1.6. Grau cohomológico de aplicações contínuas . . . . . . . . . . . . . 18

1.7. Ações sobre variedades generalizadas . . . . . . . . . . . . . . 21

CAPítulo 2. Grau e aplicações equivariantes entre variedades generalizadas 25

2.1. Introdução . . . . . . . . . . . . . . . . . . . . . . . . 25

2.2. O homomorfismo transfer para ações . . . . . . . . . . . . . . 27

2.3. Prova do teorema principal . . . . . . . . . . . . . . 30

CAPítulo 3. Grau de uma aplicação de uma variedade generalizada sobre uma esfera $\quad 35$

3.1. Introdução . . . . . . . . . . . . . . . . . . . . . . 35 
3.2. Extensão de aplicações equivariantes . . . . . . . . . . . . . . . . . . . . . . . . . . 36

3.3. Teorema principal . . . . . . . . . . . . . . . . . . . . . . . . 38

3.4. Existência de aplicações equivariantes . . . . . . . . . . . . . . 47

$\begin{array}{ll}\text { Apêndice } & 48\end{array}$

CAPítulo A. Cohomologia e co-cohomologia de feixes 49

A.1. Feixes de módulos e Família de suportes . . . . . . . . . . . . . 49

A.2. Cohomologia de feixes com suporte . . . . . . . . . . . . 56

A.3. Homologia de Borel-Moore . . . . . . . . . . . . . . . 62

CAPÍtUlo B. Notas históricas e bibliográficas 67

$\begin{array}{ll}\text { Referências Bibliográficas } & 71\end{array}$ 


\section{Introdução}

Variedades generalizadas $n$-dimensionais são espaços de Hausdorff localmente compactos cuja cohomologia ou homologia local coincide com a cohomologia ou homologia local de um espaço Euclideano $n$-dimensional. Neste trabalho, estaremos considerando as variedades generalizadas no sentido de Wilder [62] (assim como em muitos artigos a respeito desta teoria). Uma breve história sobre a origem destes espaços pode ser encontrada no Capítulo B do Apêndice.

P. Smith em [57, p. 691] criou uma classe de espaços com duas propriedades que chamou de propriedades $P$ e $Q$. Esses espaços foram posteriormente chamados variedades de Smith. Nesse mesmo artigo, ele provou que o conjunto de pontos fixos de uma aplicação periódica de potência prima sobre uma variedade topológica é uma variedade de Smith. P. Conner e E. Floyd em [18, Seção 4] provaram que as chamadas variedades de Smith coincidem com as variedades generalizadas no sentido de Wilder $[62, \text { p. 568 }]^{1}$.

O objetivo deste trabalho é estudar o grau de aplicações $G$-equivariantes entre variedades generalizadas. A presença de grupos de simetria é uma ferramenta importante que permite determinar o grau de tais aplicações.

O primeiro resultado na teoria de grau para aplicações equivariantes foi o famoso teorema de Borsuk-Ulam, o qual afirma que o grau de uma aplicação ímpar de uma esfera finito dimensional em si mesma é ímpar (vide [6] e [7]).

\footnotetext{
${ }^{1}$ É interessante notar que variedades generalizadas e variedades de Smith tenham coexistido independemente, sendo abordadas de formas distintas, por um longo período de tempo.
} 
O resultado foi primeiramente conjecturado por S. Ulam e provado por Karol Borsuk, em 1933. Desde então foram obtidas diversas generalizações deste famoso teorema para o caso de variedades topológicas, como por exemplo Yasuhiro Hara [34], Jan Jaworowski [38] e Alexander Kushkuley e Zatman Balanov [40]. Neste projeto de tese, obtivemos extensões de tais resultados para o caso de variedades generalizadas.

Em [34] e [38], Y. Hara and J. Jaworowski estudaram, independentemente, sob certas condições, o grau de aplicações equivariantes entre $G$-variedades suaves livres, onde $G$ é um grupo de Lie compacto. O principal resultado obtido por eles envolve informações fornecidas pela aplicação classificante do espaço órbita. Como principal ferramenta, eles utilizaram o homomorfismo transfer da aplicação órbita. No Capítulo 2, estudamos o grau de aplicações equivariantes entre $G$ variedades generalizadas livres sobre $\mathbb{Z}$, obtendo uma extensão dos resultados obtidos em [34] e [38].

Dado um grupo de Lie compacto $G$, consideremos o $G$-fibrado principal universal $E G \rightarrow B G$, onde $B G$ é o espaço classificante de $G$. Sejam $X$ um $G$-espaço livre paracompacto e $q_{X}: X / G \rightarrow B G$ a aplicação classificante para a $G$-ação livre sobre $X$.

Em seu trabalho [34], Y. Hara usou a definição do índice cohomológico de valor ideal, introduzido por Fadell e Husseini em [27]. O G-indice de Fadell e Husseini de $X$, denotado por $\operatorname{Ind}^{G}(X ; \mathbb{k})$, é definido como sendo o kernel do homomorfismo

$$
q_{X}^{*}: H^{*}(B G ; \mathbb{k}) \rightarrow H^{*}(X / G ; \mathbb{k}),
$$

induzido por $q_{X}$, onde $H^{*}(-; \mathbb{k})$ é a cohomologia total de Alexander-Spanier com coeficientes em algum corpo $\mathbb{k}$. Este $G$-índice é um ideal graduado no anel graduado $H^{*}(B G ; \mathbb{k})$, então para um inteiro $r$, temos

$$
\begin{aligned}
\operatorname{Ind}_{r}^{G}(X ; \mathbb{k}) & =\operatorname{Ind}^{G}(X ; \mathbb{k}) \cap H^{r}(B G ; \mathbb{k}) \\
& =\operatorname{ker}\left(q_{X}^{*}: H^{r}(B G ; \mathbb{k}) \longrightarrow H^{r}(X / G ; \mathbb{k})\right) .
\end{aligned}
$$

Por outro lado, para provar seus resultados, J. Jaworowski em [38] usou o conceito de subgrupo de $G$-classes. Este subgrupo, denotado por $\Gamma_{r}^{G}(X ; \mathbb{k})$, é definido como sendo a imagem do homomorfismo

$$
q_{X}^{*}: H^{r-\operatorname{dim} G}(B G ; \mathbb{k}) \rightarrow H^{r-\operatorname{dim} G}(X / G ; \mathbb{k}),
$$


induzido por $q_{X}$. Seus elementos são chamados $G$-classes. Consequentemente, se $f: X \rightarrow Y$ é uma aplicação $G$-equivariante, então

$$
\operatorname{Ind}_{r}^{G}(Y ; \mathbb{k}) \subset \operatorname{Ind}_{r}^{G}(X ; \mathbb{k}) \quad \text { e } \quad \Gamma_{r}^{G}(X ; \mathbb{k}) \cong(\bar{f})^{*}\left(\Gamma_{r}^{G}(Y ; \mathbb{k})\right)
$$

onde $\bar{f}: X / G \rightarrow Y / G$ é a aplicação entre os espaços órbita induzida por $f$. Observemos que em ambos casos, o homomorfismo induzido em cohomologia da aplicação classificante desempenha um papel fundamental. Nosso principal resultado será caracterizado por informações a respeito deste homomorfismo. Especificamente, provamos o seguinte

Teorema 2.1.1 [19, Theorem 1.1] Fixemos um primo p e seja $G$ um grupo de Lie compacto. Sejam $X$ e $Y$ variedades generalizadas $n$-dimensionais sobre $\mathbb{Z}$, compactas, conexas e orientadas, equipadas com uma ação livre preservando orientação do grupo G. Sejam

$$
\begin{array}{r}
q_{X}^{*}: H^{n-\operatorname{dim} G}\left(B G ; \mathbb{Z}_{p}\right) \longrightarrow H^{n-\operatorname{dim} G}\left(X / G ; \mathbb{Z}_{p}\right), \\
q_{Y}^{*}: H^{n-\operatorname{dim} G}\left(B G ; \mathbb{Z}_{p}\right) \longrightarrow H^{n-\operatorname{dim} G}\left(Y / G ; \mathbb{Z}_{p}\right),
\end{array}
$$

induzidas pela aplicação classificante para $X$ e $Y$, respectivamente. Suponhamos que $f: X \rightarrow Y$ seja uma aplicação G-equivariante.

1. Se $q_{X}^{*} \neq 0$, então $f^{*}: H^{n}\left(Y ; \mathbb{Z}_{p}\right) \rightarrow H^{n}\left(X ; \mathbb{Z}_{p}\right)$ é um isomorfismo e o grau de $f$ não é congruente a zero modulo $p$.

2. Se $q_{X}^{*}=0$ e $q_{Y}^{*} \neq 0$, então $f^{*}: H^{n}\left(Y ; \mathbb{Z}_{p}\right) \rightarrow H^{n}\left(X ; \mathbb{Z}_{p}\right)$ é trivial e o grau de $f$ é congruente a zero modulo $p$.

3. Se $q_{X}^{*}=0$ e $q_{Y}^{*}=0$, então nenhuma afirmação pode ser feita sobre o grau da aplicação $f$.

S. Eilenberg [24] estendeu o Teorema de Borsuk-Ulam à aplicações simpliciais comutando com uma ação livre simplicial de um grupo cíclico $\mathbb{Z}_{p}$ ( $p$ primo) sobre esferas. No caso das variedades topológicas e ações não necessariamente livres, uma extensão deste resultado foi provada por Alexander Kushkuley e Zatman Balanov [40], utilizando teoremas de extensão equivariante e indução sobre os tipos de órbitas. Usando tal técnica para o caso das variedades generalizadas, como resultado principal do Capítulo 3, obtivemos uma extensão do resultado principal de [40], encontrando uma relação entre os graus de duas aplicações 
equivariantes de uma variedade generalizada sobre uma esfera. Especificamente, provamos o seguinte

Teorema 3.3.1. Sejam $X$ uma variedade generalizada $n$-dimensional sobre $\mathbb{Z}$ segundo enumerável, compacta, conexa e orientada e $S=S^{n}$ a esfera $n$ dimensional. Seja $G$ um grupo finito atuando sobre $X$ e $S$ com $G$-ações concordantes. Seja $A \subset X$ um subconjunto fechado $G$-invariante. Sejam $\left(H_{1}\right),\left(H_{2}\right), \ldots,\left(H_{m}\right)$ os tipos de órbitas em $X-A$. Suponha que o conjunto $S^{H_{i}}$ seja localmente e globalmente $k$-conexo, para todo $k=0,1, \ldots, \operatorname{dim} X^{H_{i}}-1$, onde $i=1, \ldots, m$. Então, para todo par de aplicações $G$-equivariantes $\phi, \psi: X \rightarrow S$ que são $G$ equivariantemente homotópicas sobre $A$, temos

$$
\operatorname{deg}(\psi) \equiv \operatorname{deg}(\phi) \quad\left(\bmod m d c\left\{\left|G / H_{1}\right|, \ldots,\left|G / H_{m}\right|\right\}\right) .
$$

Por outro lado, Dold em [22] provou o seguinte teorema:

Se uma aplicação $f: S^{m} \rightarrow S^{n}$ comuta com alguma ação livre de um grupo não trivial finito $G$ sobre as esferas $S^{m}$ e $S^{n}$, então $n \geq m$.

Janey Daccach em seu artigo [20] generalizou este resultado, considerando a seguinte hipótese (H) sobre uma variedade topológica $M^{m}$ com uma $G$-ação livre: Suponha que $S^{m}$ possua uma $G$-ação livre e que exista uma aplicação equivariante $h: M^{m} \rightarrow S^{m}$ tal que o grau de $h$ é relativamente primo à $|G|$.

O resultado principal de J. Daccach é o seguinte

Teorema.[20, Proposition 2] Sejam $M^{m}$ e $N^{n} G$-variedades livres e suponha $M^{m}$ satisfazendo a hipótese (H). Se $f: M^{m} \rightarrow N^{n}$ é uma aplicação equivariante, então $n \geq m$.

Agora, seja $G$ um grupo finito não trivial atuando sobre uma $(\mathbb{Z}, n)$-variedade generalizada $X$ compacta, conexa e orientada com tipos de órbitas $\left(H_{1}\right), \ldots,\left(H_{l}\right)$. Dizemos que $X$ satisfaz a propriedade $(\Gamma)$ se existe uma ação de $G$ sobre a $n$-esfera $S$ tal que:

1. O conjunto $S^{H_{i}}$ é localmente e globalmente $k$-conexo para todo $k=0,1, \cdots, n_{i}$, onde $i=1, \ldots, l$ e $\operatorname{dim} X_{\left(H_{i}\right)} \leq n_{i}+1$;

2. Existe uma aplicação $G$-equivariante $\psi: X \rightarrow S$ para a qual $\operatorname{deg} \psi$ é relativamente primo a $\operatorname{mcd}\left\{\left|G / H_{i}\right|\right\}_{i=1}^{l}=\alpha(G)$. 
Utilizando a relação encontrada no Teorema 3.3.1 entre o grau de duas aplicações equivariantes provamos o seguinte

TeOrema 3.4.2 Seja $X$ uma $n$-variedade generalizada sobre $\mathbb{Z}$ segundo enumerável, compacta, conexa e orientada, com tipos de órbitas $\left(H_{1}\right), \ldots,\left(H_{l}\right)$, satisfazendo a propriedade $(\Gamma)$ e tal que $\alpha(G)>1$. Seja $Y$ um $G$-espaço métrico com tipos de órbitas $\left(D_{1}\right), \cdots,\left(D_{r}\right)$, e suponhamos que os conjuntos $S^{D_{j}}$ sejam localmente e globalmente $s$-conexos, para todo $s=0,1, \ldots, n_{j}$, com $j=1, \ldots, r$, $e \operatorname{dim} Y_{\left(D_{j}\right)} \leq n_{j}+1$. Se existe uma aplicação equivariante $f: X \rightarrow Y$, então $n \leq \operatorname{dim} Y$.

Note que a hipótese $(\Gamma)$ para uma $(\mathbb{Z}, n)$-variedade generalizada $X$ com uma $G$-ação livre se reduz à hipótese $(H)$ no teorema principal de J. Daccach em [20], ou seja, neste caso particular, $X$ satisfaz a hipótese $(\Gamma)$ se existe uma ação de $G$ sobre a n-esfera $S$ tal que existe uma aplicação $G$-equivariante $\psi: X \rightarrow S$, para a qual $\operatorname{deg} \psi$ é relativamente primo a $|G|$.

Como uma consequência do Teorema 3.4.2, considerando o caso de $G$-ações livres, obtivemos a seguinte generalização do resultado de Daccach.

Corolário 3.4.4 Sejam $X$ e $Y$ G-variedades generalizadas livres sobre $\mathbb{Z}$, compactas, conexas de dimensões $n$ e $m$ respectivamente e, suponha $X$ satisfazendo a hipótese $(\Gamma)$. Se $f: X \rightarrow Y$ é uma aplicação equivariante, então $m \geq n$. 


\section{Preliminares}

Na teoria das ações contínuas de um grupo de Lie compacto, uma das classes apropriadas de espaços topológicos é a classe das variedades generalizadas, para as quais, usando-se a cohomologia de feixes e a homologia de Borel-Moore, a Dualidade de Poincaré é válida. Para detalhes sobre a teoria de feixes, vide Capítulo A do Apêndice.

Este capítulo está organizado como segue. Nas Seções 1.1, 1.2 e 1.3, apresentamos os resultados sobre dimensão cohomológica, feixe de homologia e conexidade local em homologia. Nas Seções 1.4 e 1.5, introduzimos a construção das variedades generalizadas e seus módulos de cohomologia. As Seções 1.6 e 1.7 tratam do grau cohomológico de aplicações contínuas e suas principais propriedades e do conceito de ações sobre variedades generalizadas.

Durante todo este trabalho, tanto os módulos como as operações algébricas $\otimes$, Tor, Ext e Hom são tomadas sobre um anel $L$ com unidade 1. Todos os feixes são considerados como feixes de módulos $^{1}$ (vide Capítulo A, Subseção A.1.1 do Apêndice). Denotaremos por $H_{c}^{*}(-)$ a cohomologia de feixes com suporte compacto e por $H^{*}(-)$ a cohomologia de feixes com suporte fechado, definidos para qualquer espaço topológico ou par de espaços topólogicos (para detalhes, vide Capítulo A, Seção A.2 do Apêndice). A família de suportes $\boldsymbol{c}$ é paracompactificante se o espaço envolvido for localmente compacto; e a família de suportes $\boldsymbol{c l d}$ é paracompactificante se o espaço for paracompacto (vide Capítulo A, Subseção A.1.2 do Apêndice).

\footnotetext{
${ }^{1}$ Os feixes mais utilizados neste trabalho serão os feixes constantes e localmente constantes.
} 
DEFINIÇÃo 1.0.1. Um espaço topológico $X$ é $\mathrm{HCL}_{L}^{n}$ (singular homology locally connected) se para cada $x \in X$ e para cada vizinhança $U$ de $x$, existe uma vizinhança $V \subset U$ de $x$, dependendo de $p$, tal que a inclusão de $V$ em $U$ induz um homomorfismo trivial em homologia singular reduzida

$$
{ }_{S} \tilde{H}_{p}(V ; L) \longrightarrow{ }_{S} \tilde{H}_{p}(U ; L),
$$

para todo $p \leq n$ ( $n$ podendo ser infinito), onde ${ }_{S} \tilde{H}_{p}$ denota o $L$-módulo de homologia singular reduzido. Se $V$ é escolhido independentemente de $p, \mathrm{HCL}_{L}^{\infty}$ é denotado simplesmente por $\mathrm{HCL}_{L}$.

EXEMPLo 1.0.2. Qualquer espaço localmente contrátil e, portanto, qualquer variedade topológica (com ou sem bordo) ou CW-complexo, é HLC $\left(=\mathrm{HCL}_{\mathbb{Z}}\right)$.

ObservaÇão 1.0.3. Se $(X, A)$ é um par paracompacto HCL, então existem isomorfismos naturais $H^{*}(X, A ; L) \cong{ }_{S} H^{*}(X, A ; L) \cong{ }_{A S} H^{*}(X, A ; L)$, sendo ${ }_{S} H^{*} \mathrm{e}$ ${ }_{A S} H^{*}$, respectivamente, a cohomologia singular e a cohomologia de AlexanderSpanier (vide [14, Cap. III, Teo. 2.1, p. 187]).

Daqui em diante estaremos considerando o anel $L$ um domínio de ideais principais (PID), todos os espaços topológicos serão assumidos de Hausdorff e, salvo diga-se o contrário, localmente compactos ${ }^{1}$. Essas condições são necessárias para a definição da Homologia de Borel-Moore. Em particular, $H_{*}^{c}(-)$ e $H_{*}(-)$ denotam a homologia de Borel-Moore com suporte compacto e suporte fechado, respectivamente (vide Capítulo A, Seção A.3 do Apêndice).

ObservaÇão 1.0.4. Se $(X, A)$ é um par de espaços $\mathrm{HCL}_{L}^{\infty}$, então existe um isomorfismo natural $H_{*}^{c}(X, A ; L) \cong{ }_{S} H_{*}(X, A ; L)$, sendo ${ }_{S} H_{*}$ a homologia singular (vide [14, Cap. V, Cor. 13.6, p. 363] e o último parágrafo em [14, Cap. V, p. 288] considerando a definição de ${ }_{S} H_{*}(X, A ; L)$ encontrada em [14, Cap. V, p. 288]).

Se $A$ é fechado, temos a seguinte propriedade relacionando os módulos de (co)homologia absoluta e relativa. Para a demonstração no caso da cohomologia, vide [14, Cap. II, Prop. 12.3, p. 86] e no caso da homologia vide o último parágrafo em [14, Cap. V, p. 295] e [14, Cap. V, Cor. 5.10, p. 312].

\footnotetext{
${ }^{1}$ Os subespaços fechados ou abertos são localmente compactos (vide [48, Cap. 3, Cor. 29.3, p. 185]).
} 
Proposição 1.0.5. Se $F \subset X$ é fechado, então existe um isomorfismo natural

$$
H_{p}^{c}(X, F ; L) \cong H_{p}^{c \cap(X-F)}(X-F ; L)
$$

Além disso, para todo feixe $\mathscr{A}$, existe um isomorfismo natural

$$
H_{c}^{p}(X, F ; \mathscr{A}) \cong H_{\left.c\right|_{(X-F)} ^{p}}^{p}(X-F ; \mathscr{A})
$$

Existem dois resultados importantes com respeito a sequências exatas curtas que serão enunciados a seguir. As demonstrações podem ser encontradas em [14, eq. (9), p. 292] e em [14, Cap. II, Teo. 15.4, p. 109], respectivamente.

Proposição 1.0.6. Para todo aberto $U \subset X$, temos a sequência exata curta

$$
0 \longrightarrow \operatorname{Ext}\left(H_{c}^{p+1}(U ; L) ; L\right) \longrightarrow H_{p}(U ; L) \longrightarrow \operatorname{Hom}\left(H_{c}^{p}(U ; L) ; L\right) \longrightarrow 0
$$

Teorema dos Coeficientes Universais. Sejam $\mathscr{A}$ um feixe sobre $X$ e $M$ um módulo tal que Tor $(\mathscr{A}, M)=0$. Então, existe uma sequência exata natural

$$
0 \longrightarrow H_{c}^{n}(X ; \mathscr{A}) \otimes M \longrightarrow H_{c}^{n}(X ; \mathscr{A} \otimes M) \longrightarrow \operatorname{Tor}\left(H_{c}^{n+1}(X ; \mathscr{A}), M\right) \longrightarrow 0
$$

a qual cinde, i.e. $H_{c}^{n}(X ; \mathscr{A} \otimes M) \cong\left(H_{c}^{n}(X ; \mathscr{A}) \otimes M\right) \oplus \operatorname{Tor}\left(H_{c}^{n+1}(X ; \mathscr{A}), M\right)$.

Nossas referências principais neste capítulo serão [13], [14] e [56]. Para uma revisão histórica do surgimento das variedades generalizadas vide Capítulo B do Apêndice.

\subsection{Dimensão cohomológica}

Uma $n$-variedade topológica $M$ é um espaço de Hausdorff localmente Euclideano. O inteiro $n$ é chamado dimensão da variedade $M$, que é invariante por homeomorfismo. Uma caracterização cohomológica deste invariante numérico é que $H^{k}(M ; L)=0$, para todo $k>n$ e para toda teoria de cohomologia. Para um espaço topológico $X$ qualquer (não necessariamente localmente compacto), existem muitas generalizações desta definição, por exemplo, a dimensão topológica $\operatorname{dim} X$ (também conhecida como dimensão de Lebesgue), definida em termos de coberturas, e a $\Phi$-dimensão cohomológica $\operatorname{dim}_{\Phi, L} X$, definida para qualquer família de suportes sobre $X$. Esta última definição é baseada na caracterização 
descrita acima, i.e. $\operatorname{dim}_{\Phi, L} X$ é o maior inteiro $n($ ou $\infty)$ para o qual existe um feixe $\mathscr{A}$ sobre $X$ tal que $H_{\Phi}^{n}(X ; \mathscr{A}) \neq 0$. Para uma família de suportes $\Phi$ sobre $X, E(\Phi)$ denota a união de todos os membros de $\Phi$. Se $\Phi$ é uma família paracompactificante de suportes sobre $X$ tal que $E(\Phi)=X$, então $\operatorname{dim}_{\Phi, L} X$ torna-se independente da família de suportes $\Phi$ (vide [14, Cap. II, Prop. 16.5, p. 112]) e é chamada dimensão cohomológica de $X$ sobre $L$, denotada por $\operatorname{dim}_{L} X$ (vide [14, Cap. II, Def. 16.6, p. 112]). No nosso caso, estaremos considerando, em particular, $\Phi=c$ obtendo a seguinte

Definição 1.1.1. Seja $X$ um espaço de Hausdorff localmente compacto. A dimensão cohomológica de $X$ sobre $L$, denotada por $\operatorname{dim}_{L} X$, é definida como sendo o menor inteiro $n($ ou $\infty)$ tal que $H_{c}^{k}(X ; \mathscr{A})=0$, para todo feixe $\mathscr{A}$ sobre $X$ e para todo $k>n$. Ou, equivalentemente,

$$
\operatorname{dim}_{L} X \leq n \Longleftrightarrow H_{c}^{n+1}(U ; L)=0 \text {, para todo aberto } U \subset X
$$

Na verdade, esta última equivalência é um teorema de E. Floyd e A. Grothendieck (vide [4, Cap. I, §5] e [14, Cap.II, Teo. 16.14, p.115]) que afirma que a função $\operatorname{dim}_{L}$ sobre espaços de Hausdorff localmente compactos coincide com a função dimensão definida por Haskell Cohen [17, Def. 2.10, p. 211]. A versão homológica de dimensão e suas respectivas relações com a versão cohomológica pode ser encontrada em [35, §3].

ObservaÇão 1.1.2. Se $X$ é paracompacto, $\operatorname{dim}_{L} X \leq \operatorname{dim} X$. Quando $L=\mathbb{Z}$ e a dimensão topológica de $X$ for finita elas coincidem, mas não necessariamente coincidem se a dimensão topológica de $X$ é infinita (vide [21, Prop. 3.1, p. 549] e [14, Cap. II, p. 122]).

A seguir, apresentaremos algumas propriedades clássicas da teoria de dimensão. Para detalhes vide [14, Cap. II, Prop. 16.15, 16.8, 16.21, 16.40]. 


\section{Proposição 1.1.3.}

1. $\operatorname{dim}_{L} X \leq \operatorname{dim}_{\mathbb{Z}} X$, para todo PID L.

2. $\operatorname{dim}_{L} A \leq \operatorname{dim}_{L} X$, para todo subespaço localmente fechado $A \subset X$. Reciprocamente, se cada ponto $x \in X$ admite uma vizinhança localmente fechada $N$ com $\operatorname{dim}_{L} N \leq n$, então $\operatorname{dim}_{L} X \leq n$ (Propriedade da monotocidade).

3. $\operatorname{dim}_{L} K=0$, se e somente se, $K$ é totalmente desconexo.

4. Suponha que $X=A_{1} \cup A_{2} \cup \ldots \cup A_{i} \cup \ldots$, onde os $A_{i}$ 's são todos fechados e $\operatorname{dim}_{L} A_{i} \leq n$. Então, $\operatorname{dim}_{L} X \leq n$ (Propriedade da soma).

A igualdade em (1) é válida, em particular, quando $X=M^{n}$ é uma $n$-variedade topológica e, neste caso, $\operatorname{dim}_{L} M^{n}=\operatorname{dim}_{\mathbb{Z}} M^{n}=n$, para qualquer $L$ (vide [14, Cap. II, Cor. 16.28, p. 118]).

\subsection{Feixe de homologia}

Para entender o papel deste feixe na definição das variedades de homologia, começaremos com o caso das variedades topológicas. Para isso, suponhamos que $M$ seja uma variedade topológica $n$-dimensional e consideremos

$$
M^{0}:=\left\{\left(x, \alpha_{x}\right) \mid x \in X, \alpha_{x} \in{ }_{S} H_{n}(M, M-x ; L)\right\}
$$

Sobre este conjunto pode-se definir uma topologia tal que $p: M^{0} \rightarrow M$ definida por $p\left(x, \alpha_{x}\right)=x$ é um espaço de recobrimento. A fibra $p^{-1}(x)$ está em correspondência um a um com ${ }_{S} H_{n}(M, M-x ; L)$. Portanto, podemos ver que o par $\left(X^{0}, p\right)$ é um feixe, chamado o feixe de $L$-orientação (vide [31, Cap. 22, p. 164]). Além disso,

$$
M^{0}=\operatorname{Sheaf}\left(U \mapsto{ }_{S} H_{p}(M, M-U ; L)\right),
$$

cujas restrições do prefeixe são as induzidas em homologia singular das inclusões $(M, M-V) \subset(M, M-U)$. Este feixe é localmente constante com fibras $L$ e é constante, se $M$ for orientável (vide [14, Cap. I, Ex. 1.11, p. 7]). A. Borel e J. Moore estenderam este conceito para o caso de um espaço topológico $X$ qualquer, obtendo a seguinte

Definição 1.2.1. Para cada $p \in \mathbb{N}_{0}$ fixado, definimos o $p$-ésimo feixe de módulos de homologia local ou simplesmente o p-ésimo feixe de homologia $\mathscr{H}_{p}(X ; L)$ de $X$ 
como sendo o feixe sobre $X$

$$
\mathscr{H}_{p}(X ; L)=\operatorname{Sheaf}\left(U \mapsto H_{p}^{c}(X, X-U ; L)\right)
$$

onde as restrições $r_{U, V}: H_{p}^{c}(X, X-U ; L) \rightarrow H_{p}^{c}(X, X-V ; L)$ do prefeixe são as induzidas em homologia da inclusão $(X, X-U) \subset(X, X-V)$.

ObservaÇão 1.2.2. Bredon em [14, Cap. V, p. 293] define $\mathscr{H}_{p}(X ; L)$ como sendo o feixe sobre $X$ gerado pelo prefeixe $U \mapsto H_{p}(U ; L)$, onde suas retrições são

$$
\imath_{*}^{V, U}: H_{p}(U ; L) \rightarrow H_{p}(V ; L)
$$

definidas em [14, p. 292] para $V \subset U$. Desde que $X$ é Hausdorff e localmente compacto, podemos considerar $U$ como sendo um subconjunto aberto relativamente compacto $^{1}$ de $X$. Segue que $\boldsymbol{c} \cap U=\boldsymbol{c l d}$ e pela Proposição $1.0 .5, H_{p}^{c}(X, X-U ; L)$ é naturalmente isomorfo a $H_{p}(U ; L)$. Portanto, as duas definições coincidem e, além disso, por [14, Cap. V, Cor. 5.11, p. 313] as fibras do feixe $\mathscr{H}_{p}(X ; L)$ em $x \in X$, chamadas módulo de homologia local em $x$, são caracterizadas pelo seguinte isomorfismo:

$$
\mathscr{H}_{p}(X ; L)_{x} \cong H_{p}(X ; X-x ; L)
$$

Se $\operatorname{dim}_{L} X=n$, então $\mathscr{H}_{n}(X ; L)$ é livre de torção, desde que é gerado por $U \mapsto H_{n}(U ; L)=\operatorname{Hom}\left(H_{c}^{n}(U ; L) ; L\right)$ (segue da Proposição 1.0.6). Além disso,

$$
\mathscr{H}_{k}(X ; L)=0, \quad \text { para } \quad k>\operatorname{dim}_{L} X
$$

\subsection{Conexidade local em cohomologia}

Dualidade e a própria homologia de Borel-Moore são úteis principalmente para espaços que são razoavelmente bem comportados localmente. Por isso, no estudo homológico de certos espaços ${ }^{2}$, o conceito de conexidade local em cohomologia é muito importante. Podemos definir um invariante análogo ao $\mathrm{HCL}_{L}$ para cohomologia de feixes. Para isso, observemos que existe uma inclusão $L \hookrightarrow \Gamma(L)=$ $H^{0}(X ; L)$, que associa a cada elemento $l$ de $L$ a seção constante $l$. Chama-

\footnotetext{
${ }^{1}$ Um subconjunto é relativamente compacto se seu fecho é compacto.

${ }^{2}$ Vide por exemplo [14, Cap. II, Ex. 17.10, p. 130] e [14, Cap. V, Ex. 9.8, p. 332].
} 
mos módulos de cohomologia reduzida aos módulos $\tilde{H}^{0}(X ; L)=H^{0}(X ; L) / L$ e $\tilde{H}^{i}(X ; L)=H^{i}(X ; L)$, para $i \neq 0$. Portanto, o invariante é definido como segue.

Definição 1.3.1. Um espaço $X$ é $\operatorname{clc}_{L}^{n}$ (cohomology locally connected over $L$ ) se dados um ponto $x \in X$ e uma vizinhança $U$ de $x$, existe uma vizinhança $V \subset U$ de $x$ tal que a inclusão de $V$ em $U$ induz um homomorfismo trivial em cohomologia

$$
r_{U, V}^{*}: \tilde{H}^{p}(U ; L) \longrightarrow \tilde{H}^{p}(V ; L)
$$

para todo $p \leq n$. $X$ é $c l c_{L}^{\infty}$ se é $c l c_{L}^{n}$, para todo $n$. Se for possível escolher $V$ na definição de $c l c_{L}^{\infty}$, independentemente de $p$, diremos que $X$ é $\operatorname{clc}_{L}$.

A definição de $\operatorname{hlc}_{L}^{\infty}$ usando a homologia de Borel-Moore pode ser econtrada em [14, Cap. V, Def. 12.4, p. 350]. A condição $\operatorname{clc}_{L}^{\infty}$ é a principal propriedade de conexidade local em Topologia Algébrica do ponto de vista da teoria de feixes e possui as propriedades listadas a seguir (para detalhes vide [14, Cap. II, p. 126], [14, Cap. V, Cor. 12.10, p. 353], [14, Cap. III, Exercicio 8, p. 195] e [14, Cap. II, Cor. 17.5, p. 129]).

\section{Proposição 1.3.2.}

1. $X$ é $\operatorname{clc}_{L}^{0}$ se, e somente se, $X$ é localmente conexo.

2. Se $X$ é $\operatorname{clc}_{L}^{\infty}$ se, somente se, $X$ é hlc ${ }_{L}^{\infty}$.

3. Se $X$ é HCL, então $X$ é $\operatorname{clc}_{\mathbb{Z}}^{\infty}$.

4. Se $\operatorname{dim}_{L} X$ é finita, então a condição $c l c_{L}^{\infty}$ é equivalente a $c l c_{L}$.

A recíproca do item 3 na Proposição 1.3 .2 nem sempre é verdadeira, como mostram os exemplos em [14, Exemplo II.17.12-13] e [39]. Uma vez que estes exemplos não são métricos, é concebível (mas não pôde ser verificada) que existe uma relação estreita entre as duas condições em uma classe restrita de espaços topológicos, por exemplo, na classe de espaços completamente metrizáveis.

ObservaÇÃo 1.3.3. Aplicando o Teorema dos Coeficientes Universais [14, Teorema II.15.3] às vizinhanças compactas e usando fatos elementares sobre sequências exatas [14, Cap. II, Lema 17.3, p. 127], podemos mostrar que um espaço $\operatorname{clc}_{\mathbb{Z}}^{\infty}$ é também $\operatorname{clc}_{L}^{\infty}$, para qualquer PID $L$. 


\subsection{Variedades generalizadas}

Definição 1.4.1. Um espaço topológico $X$ localmente compacto Hausdorff é chamado uma variedade de homologia n-dimensional sobre $L$ ou, simplesmente uma $(L, n)$-variedade de homologia, se:

1. A dimensão cohomológica $\operatorname{dim}_{L} X$ é finita $(=n)$;

2. Os feixes de homologia $\mathscr{H}_{p}(X ; L)$ são nulos, para $p \neq n$, e $\mathscr{H}_{n}(X ; L)$ é livre de torção e localmente constante com fibras isomorfas a $L$.

O feixe $\mathscr{H}_{n}(X ; L)$ é chamado feixe de orientação de $X$ sobre $L$. Uma $(L, n)$ variedade de homologia $X$ diz-se $L$-orientável, se $\mathscr{H}_{n}(X ; L)$ é um feixe constante. O isomorfismo entre $\mathscr{H}_{n}(X ; L)$ e o feixe constante é chamado orientação de $X$.

A condição de que o feixe de orientação seja localmente constante significa que toda variedade de homologia é localmente orientável. Pelo isomorfismo (1.1), as condicões sobre os feixes $\mathscr{H}_{p}(X ; L)$ implicam que para todo $x \in X$

$$
H_{p}(X, X-\{x\} ; L) \cong \begin{cases}L, & \text { para } p=n \\ 0, & \text { para } p \neq n\end{cases}
$$

Se $X$ é uma $(\mathbb{Z}, n)$-variedade de homologia, por [14, Cap. V, Seq. (13), p. 294]

$$
\mathscr{H}_{p}(X ; L) \simeq 0, p \neq n \quad \text { e } \quad \mathscr{H}_{n}(X ; L) \simeq \mathscr{H}_{n}(X ; \mathbb{Z}) \otimes L
$$

Entre as muitas versões de definições de variedade generalizada, a definição a seguir foi obtida de [12, p. 461], que coincide com a noção original de Wilder, quando $L$ é um corpo (vide [4, Cap. II, Def. 2.1, p. 25 e Prop. 2.4, p. 26].

DeFINIÇÃo 1.4.2. Uma variedade generalizada $n$-dimensional sobre $L$ ou, simplesmente uma $(L, n)$-variedade generalizada, é uma $(L, n)$-variedade de homologia $X \operatorname{clc}_{L}$. $X$ é $L$-orientável, se for $L$-orientável como variedade de homologia.

ObservaÇão 1.4.3. Sejam $X$ uma $(L, n)$-variedade de homologia e $A \subset X$ um subespaço localmente fechado. Pela Proposição 1.1.3, $\operatorname{dim}_{L} A$ é finita. Desde que $\left.\mathscr{H}_{*}(A ; L) \cong \mathscr{H}_{*}(X ; L)\right|_{A}$, segue que $A$ é uma $(L, n)$-variedade de homologia. Se $X$ é $L$-orientável, então pelo isomorfismo acima, $A$ também é $L$-orientável. Se $X$ for $\operatorname{clc}_{L}$, então podemos provar que $A$ é $\operatorname{clc}_{L}$. Portanto, se $X$ é uma $(L, n)$-variedade generalizada, então $A$ também é uma $(L, n)$-variedade generalizada. 
Teorema 1.4.4. (Caracterização das variedades generalizadas) Seja $X$ um espaço localmente compacto, Hausdorff, conexo e $\operatorname{clc}_{L}^{\infty}$. São equivalentes:

1. $X$ é uma variedade generalizada $n$-dimensional sobre $L$.

2. $\operatorname{dim}_{L} X<\infty$ e, para todo $x \in X$ e $p \in \mathbb{N}, X$ satisfaz (1.3) (vide [11], [12]).

3. $\operatorname{dim}_{L} X=n<\infty$ e $\mathscr{H}_{p}(X ; L)$ é localmente constante, para cada $p$.

Se $X$ é segundo enumerável, então uma quarta condição equivalente é:

4. $\operatorname{dim}_{L} X=n<\infty$ e as fibras $\mathscr{H}_{p}(X ; L)_{x}$ são finitamente geradas e mutuamente isomorfas (i.e. independem de $x$ ) para cada $p$ (vide [12]).

Se $L$ é um corpo ou $L \cong \mathbb{Z}$ (mas $X$ não é necessariamente segundo enumerável), então uma quinta condição equivalente é:

5. $\operatorname{dim}_{L} X<\infty$ e, para todo $x \in X$ e $p \in \mathbb{N}_{0}$, (vide [11])

$$
H^{p}(X, X-\{x\} ; L) \cong \begin{cases}L, & \text { para } p=n \\ 0, & \text { para } p \neq n\end{cases}
$$

Demonstração. [14, Cap. V, Teo. 16.3, Teo. 16.8, Teo. 16.9 e Teo. 16.15].

Exemplo 1.4.5. Seja $M$ uma $n$-variedade topológica que é uma $\left(\mathbb{Z}_{2}, n\right)$-esfera de homologia, ou seja, $H_{*}\left(M ; \mathbb{Z}_{2}\right) \cong H_{*}\left(S^{n} ; \mathbb{Z}_{2}\right)$, e que não é homeomorfa à esfera $S^{n}$. Nessas condições, a suspensão de $M, S(M)$ é uma variedade generalizada $(n+1)$-dimensional sobre $\mathbb{Z}_{2}$. De fato, $S(M)$ é um CW-complexo localmente finito, $\mathrm{HCL}^{1}$ com dimensão $\operatorname{dim}_{\mathbb{Z}_{2}} S(M)$ finita. Além disso, para todo $x \in S(M)$, $H_{*}\left(S(M), S(M)-x ; \mathbb{Z}_{2}\right)$ é isomorfo a $H_{*}\left(\mathbb{R}^{n+1}, \mathbb{R}^{n+1}-0 ; \mathbb{Z}_{2}\right)$. O resultado segue da Observação 1.0.4, Proposição 1.3 .2 e do Teorema 1.4.4. Porém, $S(M)$ não é variedade topológica. De fato, observemos que dada qualquer vizinhança $V$ do ponto $x_{0} \in S(M)$, temos que $V$ é homeomorfa ao cone de $M$, porém $C(M)$ não é homeomorfo a um disco, uma vez que $\partial(C M)=M$ e $M$ não é homeomorfa à esfera $S^{n}$.

Exemplo 1.4.6. Outro exemplo pode ser construído como uma soma conexa infinita $L_{q} \# L_{q} \# L_{q} \# \cdots$, onde $L_{q}$ é um espaço lens $n$-dimensional, com $\pi_{1}\left(L_{q}\right)=$ $\mathbb{Z}_{q}$ e $q$ um primo ímpar. Este espaço na verdade é uma variedade generalizada $n$-dimensional sobre $\mathbb{Z}_{q}, q$ primo ímpar (vide [37, p. 157]).

\footnotetext{
${ }^{1} \mathrm{CW}$-complexos são localmente contráteis.
} 
Proposição 1.4.7. Se $X$ é uma $n$-dimensional variedade generalizada sobre $\mathbb{Z}$ (orientável), então $X$ é uma $n$-dimensional variedade generalizada sobre $L$ (orientável), para todo PID L.

Demonstração. Pela Observação 1.4.3, cada componente conexa $C$ de $X$ é uma $(\mathbb{Z}, n)$-variedade generalizada. Segue da Proposição 1.1.3, Observação 1.3 .3 e dos isomorfismos em (1.4), que $\operatorname{dim}_{L} C<\infty, C$ é $\operatorname{clc}_{L}, \mathscr{H}_{n}(C ; L)_{x} \cong L$ e $\mathscr{H}_{p}(C ; L)_{x} \cong$ 0 , para $p \neq n$. Então, pelo Teorema 1.4.4, cada componente conexa $C$ de $X$ é uma $(L, n)$-variedade generalizada. O resultado segue do fato de $X$ ser a soma topológica de suas componentes conexas. A orientabilidade segue do isomorfismo (1.4).

Quando o espaço for primeiro enumerável, a condição de ser clc na Definição 1.4.2 é automática ${ }^{1}$. Este resultado foi provado em [12, Teo. 2.1, p. 464] (vide também [14, Cap. V, Teo. 16.13, p. 379]). Para o caso em que $L$ é um corpo e para um PID enumerável, isso foi provado por W. Mitchell [47, Teo. 1, p. 348] (vide também [35, Teo. 8, p. 343]). Especificamente, temos a seguinte

Proposição 1.4.8. Seja $X$ uma variedade de homologia $n$-dimensional sobre $L$. Se $X$ é primeiro enumerável, $L$ é um PID enumerável ou um corpo, então $X$ é $\operatorname{clc}_{L}$ e, portanto, uma variedade generalizada $n$-dimensional sobre $L$.

DeFinição 1.4.9. Um espaço $X$ é chamado uma variedade generalizada $n$-dimensional sobre $L$ com bordo $\partial X$, se $\partial X \subset X$ é fechado, $X-\partial X$ é uma $(L, n)$ variedade generalizada, $\partial X$ é uma $(L, n-1)$-variedade generalizada e os feixes de homologia $\mathscr{H}_{n}(X ; L)$ se anulam sobre $\partial X$.

\subsection{Módulos de cohomologia de variedades generalizadas}

Borel e Moore construíram, para espaços de Hausdorff locamente compactos $X$ de dimensão cohomológica finita, uma sequência espectral em que

$$
E_{2}^{p, q}=H_{c}^{p}\left(X ; \mathscr{H}_{-q}(X ; \mathscr{A})\right) \Longrightarrow H_{-p-q}^{c}(X ; \mathscr{A})
$$

definida para todo feixe $\mathscr{A}$ de módulos e para toda família $\Phi$ de suportes paracompactificante, a qual colapsa e dá origem à dualidade de Poincaré para variedades de homologia (vide [14, Cap. V, Teo. 9.2, p. 329] ou [5, Teo. 7.6, p. 153]).

\footnotetext{
${ }^{1}$ Assim, é plausível conjecturar que uma variedade de homologia é necessariamente clc e, portanto, é uma variedade generalizada.
} 
Teorema da Dualidade de Poincaré. Sejam $X$ uma $(L, n)$-variedade de homologia e $\mathscr{A}$ um feixe sobre $X$. Então, para todo inteiro $p$, existe um isomorfismo natural

$$
\Delta: H_{c}^{p}\left(X ; \mathscr{H}_{n}(X ; L) \otimes \mathscr{A}\right) \stackrel{\cong}{\longrightarrow} H_{n-p}^{c}(X ; \mathscr{A}) .
$$

Segue de $\left[9\right.$, Cor. 3.4, p. 327] que $\mathscr{H}_{n}(X ; L) \otimes \mathscr{H}_{n}(X ; L)$ é o feixe constante $L$. Seja $X$ uma $(L, n)$-variedade generalizada conexa. Pelo Teorema da Dualidade de Poincaré,

$$
H_{c}^{n}\left(X ; \mathscr{H}_{n}(X ; L)\right) \cong H_{0}^{c}\left(X ; \mathscr{H}_{n}(X ; L) \otimes \mathscr{H}_{n}(X ; L)\right) \cong H_{0}^{c}(X ; L) \cong L .
$$

O último isomorfismo segue de [14, Cap. V, Teo. 5.14, p. 314]. Portanto, temos os seguintes módulos de cohomologia de uma $(L, n)$-variedade generalizada.

Proposição 1.5.1. Seja $X$ uma variedade generalizada $n$-dimensional sobre $L$ conexa e $Y$ uma variedade generalizada $n$-dimensional sobre $L$ conexa com bordo $\partial Y$. Então,

1. $H_{c}^{n}\left(X ; \mathscr{H}_{n}(X ; L)\right) \cong L$.

2. $H_{c}^{n}\left(Y, \partial Y ; \mathscr{H}_{n}(Y ; L)\right) \cong L$.

3. $X$ é orientável se, e somente se, $H_{c}^{n}(X ; L) \cong L$.

Demonstração. O item 2, segue da Proposição 1.0.5. Para a prova do item 3, vide [14, Cap. V, Teo. 16.16(f), p. 381].

Denotaremos o $L$-módulo $\mathscr{H}_{n}(X ; L)$ simplesmente por $\mathscr{H}_{n}(X)$, quando $L=\mathbb{Z}$. Um elemento do grupo $H_{c}^{n}\left(X ; \mathscr{H}_{n}(X)\right)$ correspondente a 1 ou -1 de $\mathbb{Z}$ é chamado classe de cohomologia fundamental de $X$. A escolha desta classe é equivalente à escolha de uma orientação local de $X$ no seguinte sentido: considere $U$ um aberto conexo tal que $\mathscr{H}_{n}(U)=\left.\mathscr{H}_{n}(X)\right|_{U} \cong U \times \mathbb{Z}$ (orientabilidade local de $X$ ). Entenderemos como uma orientação local de $X$ o produto de um gerador de $\mathbb{Z}$ com uma orientação ordinária de $U$ (a escolha de um gerador de $H_{c}^{n}(U ; \mathbb{Z}$ ), já que $U$ é uma $(\mathbb{Z}, n)$-variedade generalizada orientável). Assim, diremos que $X$ é localmente orientada quando escolhemos uma orientação local para $X$, ou seja uma classe de cohomologia. Analogamente, uma classe de cohomologia fundamental com respeito a $L$, significa a escolha de um dos geradores 1 ou -1 deste anel. No caso em que $-1 \neq 1$, a escolha de uma classe de cohomologia fundamental com respeito 
a $L$ é equivalente à escolha de uma orientação local de $X$, como anteriormente (para detalhes, vide [55]).

\subsection{Grau cohomológico de aplicações contínuas}

Seja $f: X \rightarrow Y$ uma aplicação própria entre entre $(\mathbb{Z}, n)$-variedades generalizadas e consideremos o homomorfismo induzido em cohomologia

$$
f_{c}^{*}: H_{c}^{p}\left(Y ; \mathscr{H}_{n}(Y)\right) \longrightarrow H_{c}^{p}\left(X ; f^{*} \mathscr{H}_{n}(Y)\right)
$$

Se $f^{*} \mathscr{H}_{n}(Y) \simeq \mathscr{H}_{n}(X)$, então $f_{c}^{*}(1)$ é um inteiro, caso contrário, temos por [14, Cap. V, Teo. $16.16(\mathrm{~g})$, p. 381], que $H_{c}^{p}\left(X ; f^{*} \mathscr{H}_{n}(Y)\right) \cong \mathbb{Z}_{2}$, ou seja, $f_{c}^{*}(1)$ é 0 ou 1. Este valor é chamado o grau módulo 2 (vide [51], [26] e [56]). Segundo a terminologia de De Rham e E.G. Sklyarenko (vide, por exemplo, [56, p. 1689]) temos a seguinte

Definição 1.6.1. Sejam $X$ e $Y$ variedades generalizadas $n$-dimensionais sobre $L$. Dizemos que uma aplicação contínua $f: X \rightarrow Y$ é L-orientável se

$$
f^{*} \mathscr{H}_{n}(Y ; L) \simeq \mathscr{H}_{n}(X ; L)
$$

Se $L=\mathbb{Z}$, dizemos simplesmente que $f$ é orientável.

ObSERVAÇão 1.6.2. Se $X$ e $Y$ são $(L, n)$-variedades generalizadas $L$-orientáveis, então os feixes de orientação $\mathscr{H}_{n}(X ; L)$ e $\mathscr{H}_{n}(Y ; L)$ são isomorfos ao feixe constante $L$. Desde que o funtor $f^{*}: \mathcal{S h}(Y) \rightarrow \mathcal{S h}(X)$ preserva feixes constantes, ou seja, $f^{*}(Y \times M) \simeq X \times M$, para todo módulo $M$, temos que toda aplicação contínua $f: X \rightarrow Y$ é $L$-orientável.

A seguir, apresentaremos a definição de grau cohomológico na versão das variedades generalizadas, considerando o homomorfismo (1.5) e a Definição 1.6.1.

DeFinição 1.6.3. Seja $f: X \rightarrow Y$ uma aplicação própria e orientável entre variedades generalizadas $n$-dimensionais sobre $\mathbb{Z}$, conexas e localmente orientadas. Definimos o grau de $f$ pelo inteiro $\operatorname{deg}(f)$ dado por

$$
\operatorname{deg} f:=f_{c}^{*}(1) \in \mathbb{Z},
$$


onde $f_{c}^{*}: \mathbb{Z} \cong H_{c}^{n}\left(Y ; \mathscr{H}_{n}(Y)\right) \longrightarrow H_{c}^{n}\left(X ; \mathscr{H}_{n}(X)\right) \cong \mathbb{Z}$ é a induzida em cohomologia e $1 \in \mathbb{Z}$. Ou seja, se $[X]$ e $[Y]$ são as classes de cohomologia fundamentais de $X$ e $Y$, respectivamente, então o grau de $f$ é o inteiro $\operatorname{deg}(f)$ tal que

$$
f_{c}^{*}([Y])=\operatorname{deg} f \cdot[X] .
$$

Observação 1.6.4. Podemos também definir o grau de $f$ sobre $L$, denotado por $\operatorname{deg}_{L}(f)$, tomando valores no anel $L$, de maneira semelhante à definição anterior, via a cohomologia $H_{c}^{n}\left(-; \mathscr{H}_{n}(-; L)\right)$. Em particular, quando $L=\mathbb{Z}, \operatorname{deg}_{\mathbb{Z}}(f)=$ $\operatorname{deg}(f)$. De maneira análoga, pode-se definir o mesmo invariante para o caso das variedades generalizadas com bordo (vide [56, p. 1700]).

Proposição 1.6.5. [56, Prop. 4.3, p. 1704]. Seja $f: X \rightarrow Y$ uma aplicação própria e orientável entre variedades generalizadas $n$-dimensionais sobre $\mathbb{Z}$, conexas e localmente orientadas. Se $\operatorname{deg} f \neq 0$, então $f$ é sobrejetora.

Pela Proposição 1.4.7 temos a seguinte

Proposição 1.6.6. [56, Teo. 3.2, p. 1700]. Seja $f: X \rightarrow Y$ uma aplicação própria e orientável entre variedade generalizadas $n$-dimensionais sobre $\mathbb{Z}$, conexas e localmente orientadas. Então, $f$ é L-orientável para qualquer PID L. Além disso,

$$
\operatorname{deg}_{L} f=\operatorname{deg} f \cdot 1 \in L,
$$

onde $1 \in L$.

Sabemos que para coeficientes constantes a cohomologia de feixes satisfaz o Axioma da Invariância por Homotopia para aplicações propriamente homotópi$\operatorname{cas}^{1}$ (vide [14, Cap. II, Teo. 11.12, p. 80]). Veremos a seguir que este resultado também é válido para feixes localmente constantes. Sejam $f, g: X \rightarrow Y$ aplicações próprias e propriamente homotópicas entre espaços de Hausdorff localmente compactos. Seja $\mathscr{B}$ um feixe localmente constante sobre $Y$. Desde que a imagem inversa de um feixe localmente constante é invariante por homotopia, $f^{*} \mathscr{B} \simeq g^{*} \mathscr{B}$. Seja $F: X \times I \rightarrow Y$ a homotopia própria entre $f$ e $g$. Esta homotopia induz sobre $X \times I$ o feixe $F^{*} \mathscr{B}=f^{*} \mathscr{B} \times I=g^{*} \mathscr{B} \times I$. Segue de [14, Cap. II, Teo. 11.9, p. 79], que a aplicação $\imath_{t}: X \rightarrow X \times I$, dada pela inclusão $x \rightarrow(x, t)$, induz um isomorfismo de cohomologias $\imath_{t}^{*}: H_{c}^{*}\left(X \times I ; F^{*} \mathscr{B}\right) \rightarrow H_{c}^{*}\left(X ; f^{*} \mathscr{B}\right)=H_{c}^{*}\left(X ; g^{*} \mathscr{B}\right)$,

\footnotetext{
${ }^{1}$ Quando existe uma homotopia própria $F: X \times I \rightarrow Y$ entre $f$ e $g$.
} 
o qual é independente de $t$. Em particular, $\imath_{0}^{*}=\imath_{1}^{*}$. Então,

$$
f_{c}^{*}=\left(F \circ \imath_{0}\right)_{c}^{*}=\imath_{0}^{*} \circ F_{c}^{*}=\imath_{1}^{*} \circ F_{c}^{*}=\left(F \circ \imath_{1}\right)_{c}^{*}=g_{c}^{*} .
$$

Portanto, temos o seguinte

Teorema 1.6.7. Sejam $f, g: X \rightarrow Y$ aplicações próprias, $L$-orientáveis e propriamente homotópicas entre variedades generalizadas $n$-dimensionais sobre $\mathbb{Z}$, conexas e localmente orientadas. Então,

$$
\operatorname{deg}_{L}(f)=\operatorname{deg}_{L}(g)
$$

As propriedades a seguir são consequências imediatas das Proposições A.2.5 e A.2.7 (vide Capítulo A, Seção A.2 do Apêndice).

Proposição 1.6.8. Seja $X$ uma variedade generalizada $n$-dimensional sobre $L$, conexa e localmente orientada. A aplicação identidade Id : $X \rightarrow X$ é sempre orientável sobre L. Além disso,

$$
\operatorname{deg}_{L}(\mathrm{Id})=1
$$

Proposição 1.6.9. Sejam $f: X \rightarrow Y$ e $g: Y \rightarrow Z$ aplicações próprias e $L$-orientáveis entre variedades generalizadas $n$-dimensionais sobre $L$, conexas e localmente orientadas. Então, $g \circ f$ é uma aplicação própria e $L$-orientável. Além disso,

$$
\operatorname{deg}_{L}(g \circ f)=\operatorname{deg}_{L}(f) \cdot \operatorname{deg}_{L}(g)
$$

Em particular, se $f$ é um homeomorfismo, $\operatorname{deg}_{\mathbb{Z}}(f)= \pm 1$.

Seja $X$ uma $(L, n)$-variedade generalizada conexa e localmente orientada. Desde que $\mathscr{H}_{n}(Y ; L)$ é localmente constante com fibras isomorfas a $L$, sejam $y \in Y$ e $U \subset Y$ uma vizinhança aberta e conexa suficientemente pequena de $y$ tal que $\left.\mathscr{H}_{n}(Y ; L)\right|_{U} \simeq U \times L$. Logo, o conjunto aberto $V=f^{-1}(U)$ pode ser representado como a união de suas componentes conexas $V=\bigcup_{i} V_{i}$. Seja $f_{i}$ a restrição de $f$ ao conjunto $V_{i}$. Podemos ver que as aplicações $f_{i}: V_{i} \rightarrow U$ são próprias e desde que o feixe $\left.\mathscr{H}_{n}(Y ; L)\right|_{U}=\mathscr{H}_{n}(U ; L)$ é constante, os graus $\operatorname{deg}_{L}\left(f_{i}\right)$ são definidos (vide Observação 1.6.2). Portanto, temos a seguinte

Proposição 1.6.10. [56, Proposição 4.10, p. 1707]. Os graus $\operatorname{deg}_{L}\left(f_{i}\right)$ são não 
nulos somente para uma quantidade finita de $V_{i}$. Além disso,

$$
\operatorname{deg}_{L} f=\sum_{i} \operatorname{deg}_{L} f_{i}
$$

se a aplicação $f$ é L-orientável.

Também podemos definir o grau homológico, o qual está relacionado com a versão cohomológica definida anteriormente. Para detalhes, vide [56, p. 1702].

\subsection{Ações sobre variedades generalizadas}

Um grupo de transformações é uma tripla $(G, X, \mu)$, onde $G$ é um grupo topológico, $X$ é um espaço topológico de Hausdorff e $\mu: G \times X \rightarrow X, \mu(g, x)=g x$ é uma ação de $G$ sobre $X$. O espaço $X$ é chamado um $G$-espaço. Uma aplicação $G$-equivariante é uma aplicação contínua $f: X \rightarrow Y$ entre dois $G$-espaços que preserva a $G$-ação, ou seja, $f(g x)=g f(x)$, para qualquer $x \in X$ e $g \in G$.

Sejam $X$ um $G$-espaço e $x \in X$. O subgrupo de isotropia $G_{x}$ e a órbita $G(x)$ de $x$ são definidos como o subgrupo de $G$ e o subespaço de $X$ dados por:

$$
G_{x}=\{g \in G \mid g x=x\}, \quad G(x)=\{g x \in X \mid g \in G\}
$$

respectivamente. Denotamos por $X / G$ o conjunto das órbitas da $G$-ação. Seja $\pi: X \rightarrow X / G$ a aplicação órbita definida por $\pi(x)=G(x)$. O espaço quociente $X / G$ é chamado espaço órbita. Uma aplicação $G$-equivariante $f: X \rightarrow Y$ induz uma aplicação contínua entre os espaços órbita $\bar{f}: X / G \rightarrow Y / G$.

Definição 1.7.1. Uma ação de $G$ sobre $X$ é chamada livre se $G_{x}$ é trivial, para todo $x \in X$; e semi-livre se $G_{x}$ é trivial ou $G_{x}$ coincide com $G$, para todo $x \in X$.

Seja $H$ um subgrupo de $G$. Uma classe de conjugados de $H$ é o conjunto $\left\{g \mathrm{Hg}^{-1} \mid g \in G\right\}$. O conjunto dos pontos fixos de $H$ sobre $X$ é definido como sendo o subespaço

$$
X^{H}=\{x \in X \mid h x=x, \forall h \in H\} .
$$

ObservaÇão 1.7.2. O quociente $W(H)=N(H) / H$ é chamado grupo Weyl de $H$, onde $N(H)=\{n \in G \mid n H=H n\}$ é o normalizador de $H$ em $G$. Podemos ver que $W(H)$ atua sobre $X^{H}$ da seguinte forma:

$$
W(H) \times X^{H} \rightarrow X^{H}, \quad(n H) x=n x .
$$


Esta $W(H)$-ação é livre sobre o subespaço $X_{H}=\left\{x \in X \mid G_{x}=H\right\}$.

Vamos agora restringir a nossa atenção exclusivamente para ações de um grupo de Lie compacto. Começamos listando as seguintes propriedades ([13, Cap. I, Teo. 3.1, Prop. 4.1]). Seja $G$ um grupo de Lie compacto atuando sobre $X$. Então,

1. A aplicação órbita $\pi: X \rightarrow X / G$ é própria e fechada.

2. Para cada $x \in X$, a órbita $G(x)$ é homeomorfa a $G / G_{x}$.

Seja $O$ uma órbita de $X$. O tipo de órbita de $O$ é a classe de conjugados de $H \leq G$ tal que $O$ é homeomorfa a $G / H$. Desde que $G_{g x}=g G_{x} g^{-1}$, para todo $g \in G$, os grupos de isotropia em cada ponto da órbita $G(x)$ formam uma classe de cojugados de $G_{x}$. Portanto, para grupos de Lie compactos, usando a propriedade (2) descrita acima, temos a seguinte

Definição 1.7.3. Sejam $G$ um grupo de Lie compacto. O tipo de órbita $(H)$ do $G$-espaço $X$ é a classe de conjugados de algum subgrupo de isotropia $H$. Chamaremos fibrado órbita ao subespaço

$$
X_{(H)}:=\left\{x \in X \mid\left(G_{x}\right)=(H)\right\}
$$

Se $H$ é conjugado a um subgrupo de $K \leq G$, escreveremos $(H) \leq(K)$. Portanto, o conjunto $\operatorname{Or}(X)$ de todos os tipos de órbitas é parcialmente ordenado.

Proposição 1.7.4. [13, Cap. II, Teo. 5.8, p. 88]. Seja G um grupo de Lie compacto agindo sobre um espaço de Hausdorff localmente compacto $X$. Se todas as órbitas têm tipo $(H)$, então a aplicação órbita $\pi: X \rightarrow X / G$ é um fibrado com fibra $G / H$ e grupo de estrutura $W(H)$.

Sejam $X$ e $Y(\mathbb{Z}, n)$-variedades generalizadas conexas. Suponha que um grupo de Lie compacto atue sobre $X$ e $Y$. Pela Proposição 1.6.9 cada elemento $g$ de $G$, visto como um homeomorfismo $g: X \rightarrow X, x \mapsto g x$, tem grau \pm 1 (analogamente para $Y$ ). Portanto, essas ações definem os chamados homomorfismos orientação $e_{G, X}: G \rightarrow \mathbb{Z}^{*}=\{1,-1\}$ e $e_{G, Y}: G \rightarrow \mathbb{Z}^{*}=\{1,-1\}$.

DefiniÇÃo 1.7.5. Sejam $X$ e $Y$ variedades generalizadas $n$-dimensional sobre $\mathbb{Z}$ conexas. As ações de um grupo de Lie compacto $G$ sobre $X$ e $Y$ são chamadas concordantes se $e_{G, X}=e_{G, Y}$. 
Se $f: X \rightarrow Y$ é uma aplicação $G$-equivariante, então para todo $g \in G$, $f \circ g=g \circ f$. Se $f$ é orientável, temos pela Proposição 1.6 .9

$$
\operatorname{deg}(f)=e_{G, X}\left(g^{-1}\right) \operatorname{deg}(f) e_{G, Y}(g)
$$

Agora, se $e_{G, X}(h) \neq e_{G, Y}(h)$, para algum $h \in G$, então $\operatorname{deg}(f)=-\operatorname{deg}(f)$, e segue que $\operatorname{deg}(f)=0$. Assim, sempre que nos referirmos ao grau de aplicações $G$-equivariantes, assumiremos que as ações são concordantes.

OBSERVAÇÃo 1.7.6. A condição das ações serem concordantes significa que as ações preservam ou invertem as orientações sobre $X$ e $Y$, simultaneamente. 


\section{Grau e aplicações equivariantes entre variedades generalizadas}

\subsection{Introdução}

Em [34] e [38], Y. Hara and J. Jaworowski estudaram, independentemente, sob certas condições, o grau de aplicações equivariantes entre $G$-variedades suaves livres, onde $G$ é um grupo de Lie compacto. O principal resultado obtido por eles envolve informações fornecidas pela aplicação classificante do espaço órbita. Como principal ferramenta, eles utilizaram o homomorfismo transfer da aplicação órbita. Neste capítulo, estudamos o grau de aplicações equivariantes entre $G$ variedades generalizadas livres sobre $\mathbb{Z}$, obtendo uma extensão dos resultados obtidos em [34] e [38].

Dado um grupo de Lie compacto $G$, consideremos o $G$-fibrado principal universal $E G \rightarrow B G$, onde $B G$ é o espaço classificante de $G$. Sejam $X$ um $G$-espaço livre paracompacto e $q_{X}: X / G \rightarrow B G$ a aplicação classificante para a $G$-ação livre sobre $X$.

Em seu trabalho [34], Y. Hara usou a definição do índice cohomológico de valor ideal, introduzido por Fadell e Husseini em [27]. O G-indice de Fadell e Husseini de $X$, denotado por $\operatorname{Ind}^{G}(X ; \mathbb{k})$, é definido como sendo o kernel do homomorfismo

$$
q_{X}^{*}: H^{*}(B G ; \mathbb{k}) \rightarrow H^{*}(X / G ; \mathbb{k}),
$$

induzido por $q_{X}$, onde $H^{*}(-; \mathbb{k})$ é a cohomologia total de Alexander-Spanier com coeficientes em algum corpo $\mathbb{k}$. Este $G$-índice é um ideal graduado no anel gra- 
duado $H^{*}(B G ; \mathbb{k})$, então para um inteiro $r$, temos

$$
\begin{aligned}
\operatorname{Ind}_{r}^{G}(X ; \mathbb{k}) & =\operatorname{Ind}^{G}(X ; \mathbb{k}) \cap H^{r}(B G ; \mathbb{k}) \\
& =\operatorname{ker}\left(q_{X}^{*}: H^{r}(B G ; \mathbb{k}) \longrightarrow H^{r}(X / G ; \mathbb{k})\right) .
\end{aligned}
$$

Por outro lado, para provar seus resultados, J. Jaworowski em [38] usou o conceito de subgrupo de G-classes. Este subgrupo, denotado por $\Gamma_{r}^{G}(X ; \mathbb{k})$, é definido como sendo a imagem do homomorfismo

$$
q_{X}^{*}: H^{r-\operatorname{dim} G}(B G ; \mathbb{k}) \rightarrow H^{r-\operatorname{dim} G}(X / G ; \mathbb{k}),
$$

induzido por $q_{X}$. Seus elementos são chamados $G$-classes. Consequentemente, se $f: X \rightarrow Y$ é uma aplicação $G$-equivariante, então

$$
\operatorname{Ind}_{r}^{G}(Y ; \mathbb{k}) \subset \operatorname{Ind}_{r}^{G}(X ; \mathbb{k}) \quad \text { e } \quad \Gamma_{r}^{G}(X ; \mathbb{k}) \cong(\bar{f})^{*}\left(\Gamma_{r}^{G}(Y ; \mathbb{k})\right)
$$

onde $\bar{f}: X / G \rightarrow Y / G$ é a aplicação entre os espaços órbita induzida por $f$. Observemos que em ambos casos, o homomorfismo induzido em cohomologia da aplicação classificante desempenha um papel fundamental. Nosso principal resultado será caracterizado por informações a respeito deste homomorfismo. Especificamente, neste capítulo, provaremos o seguinte:

Teorema 2.1.1. [19, Theorem 1.1] Fixemos um primo $p$ e seja $G$ um grupo de Lie compacto. Sejam $X$ e $Y$ variedades generalizadas $n$-dimensionais sobre $\mathbb{Z}$, compactas, conexas e orientadas, equipadas com uma ação livre preservando orientação do grupo G. Sejam

$$
\begin{gathered}
q_{X}^{*}: H^{n-\operatorname{dim} G}\left(B G ; \mathbb{Z}_{p}\right) \longrightarrow H^{n-\operatorname{dim} G}\left(X / G ; \mathbb{Z}_{p}\right), \\
q_{Y}^{*}: H^{n-\operatorname{dim} G}\left(B G ; \mathbb{Z}_{p}\right) \longrightarrow H^{n-\operatorname{dim} G}\left(Y / G ; \mathbb{Z}_{p}\right),
\end{gathered}
$$

induzidas pela aplicação classificante para $X$ e $Y$, respectivamente. Suponhamos que $f: X \rightarrow Y$ seja uma aplicação $G$-equivariante. Então, temos o seguinte:

1. Se $q_{X}^{*} \neq 0$, então $f^{*}: H^{n}\left(Y ; \mathbb{Z}_{p}\right) \rightarrow H^{n}\left(X ; \mathbb{Z}_{p}\right)$ é um isomorfismo e o grau de $f$ não é congruente a zero modulo $p$.

2. Se $q_{X}^{*}=0$ e $q_{Y}^{*} \neq 0$, então $f^{*}: H^{n}\left(Y ; \mathbb{Z}_{p}\right) \rightarrow H^{n}\left(X ; \mathbb{Z}_{p}\right)$ é trivial e o grau de $f$ é congruente a zero modulo $p$. 
3. Se $q_{X}^{*}=0$ e $q_{Y}^{*}=0$, então nenhuma afirmação pode ser feita sobre o grau da aplicação $f$.

Corolário 2.1.2. Fixemos um primo p e seja $G$ um grupo de Lie compacto. Sejam $X$ e $Y$ variedades generalizadas $n$-dimensionais sobre $\mathbb{Z}$, compactas, conexas e orientadas, equipadas com uma ação livre preservando orientação do grupo $G$. Seja $f: X \rightarrow Y$ uma aplicação $G$-equivariante.

1. Se

$$
\operatorname{Ind}_{n-\operatorname{dim} G}^{G}\left(X ; \mathbb{Z}_{p}\right) \neq H^{n-\operatorname{dim} G}\left(B G ; \mathbb{Z}_{p}\right), \quad \text { ou } \quad \Gamma_{n}^{G}\left(X ; \mathbb{Z}_{p}\right) \neq 0
$$

então $f^{*}: H^{n}\left(Y ; \mathbb{Z}_{p}\right) \rightarrow H^{n}\left(X ; \mathbb{Z}_{p}\right)$ é um isomorfismo e o grau de $f$ não é congruente a zero módulo $p$.

2. Se

$$
\begin{gathered}
\operatorname{Ind}_{n-\operatorname{dim} G}^{G}\left(Y, \mathbb{Z}_{p}\right) \varsubsetneqq \operatorname{Ind}_{n-\operatorname{dim} G}^{G}\left(X ; \mathbb{Z}_{p}\right)=H^{n-\operatorname{dim} G}\left(B G ; \mathbb{Z}_{p}\right), \\
\text { ou } \quad \Gamma_{n}^{G}\left(X ; \mathbb{Z}_{p}\right)=0 \text { e } \quad \Gamma_{n}^{G}\left(Y ; \mathbb{Z}_{p}\right) \neq 0
\end{gathered}
$$

então $f^{*}: H^{n}\left(Y ; \mathbb{Z}_{p}\right) \rightarrow H^{n}\left(X ; \mathbb{Z}_{p}\right)$ é trivial e o grau de $f$ é congruente a zero modulo $p$.

\subsection{O homomorfismo transfer para ações}

Seja $G$ um grupo de Lie compacto atuando sobre um espaço paracompacto $X$. Desde que a aplicação órbita $\pi: X \rightarrow X / G$ é uma aplicação fechada e própria, temos por [14, Capítulo IV, Proposição 4.2, p. 214], que o feixe de Leray $\mathscr{H}^{t}(\pi ; L)$ de $\pi$, que é o feixe gerado pelo prefeixe

$$
U \mapsto H^{t}\left(\pi^{-1}(U) ; L\right)
$$

tem fibras

$$
\mathscr{H}^{t}(\pi ; L)_{\breve{x}} \cong H^{t}\left(\pi^{-1}(\breve{x}) ; L\right)=H^{t}(G x ; L),
$$

para todo $\breve{x} \in X / G$. Para coeficientes no feixe constante $L$ e para suportes fechados em $X$ e $X / G$, existe uma sequência espectral de Leray, para $\pi$ [14, Capítulo IV, Teorema 6.1, p. 222], a qual tem como $E_{2}$-termo

$$
E_{2}^{s, t}=H^{s}\left(X / G ; \mathscr{H}^{t}(\pi ; L)\right)
$$


e converge para $H^{s+t}(X ; L)$.

Nosso propósito, nesta seção, é apresentar a construção do homomorfismo transfer para qualquer grupo de Lie compacto $G$ :

$$
\tau_{X}: H^{s+\operatorname{dim} G}(X ; L) \longrightarrow H^{s}(X / G ; L) .
$$

Para definir o homomorfismo transfer, escolhemos uma orientação do grupo $G$ e da sua componente conexa $G_{0}$ da identidade em $G$, ou seja, geradores de $H^{\operatorname{dim} G}(G, L)$ e $H^{\operatorname{dim} G}\left(G_{0}, L\right)$, respectivamente. Fixaremos estes geradores em todo este capítulo.

Caso finito: Para este caso, A. Borel definiu o homomorfismo transfer em [4, Capítulo III, Seção 2] (vide também [14, Capítulo II, Seção 19]). Suponhamos que $G$ seja finito. Neste caso, $\operatorname{dim} G=0$ e desde que $G x$ é finito, $H^{t}(G x ; L)=0$, para todo $t \neq 0$ e, portanto, $\mathscr{H}^{t}(\pi ; L)=0$, para todo $t \neq 0$. Então, $E_{2}^{s, t}=0$, para $t \neq 0$ e, assim,

$$
H^{s}(X ; L) \stackrel{\cong}{\rightrightarrows} E_{\infty}^{s, 0} \stackrel{\cong}{\longmapsto} E_{2}^{s, 0}=H^{s}\left(X / G ; \mathscr{H}^{0}(\pi ; L)\right) .
$$

Para cada $g \in G$, considerado como um homeomorfismo $g: X \rightarrow X$, temos que $\pi \circ g=\pi$ e, consequentemete, $g: \pi^{-1}(U) \rightarrow \pi^{-1}(U)$ também é um homeomorfismo. Logo, o isomorfismo $g^{*}: H^{0}\left(\pi^{-1}(U) ; L\right) \rightarrow H^{0}\left(\pi^{-1}(U) ; L\right)$ induz uma ação sobre $H^{0}\left(\pi^{-1}(U) ; L\right)$ e, portanto, sobre o feixe de Leray $\mathscr{H}^{0}(\pi ; L)$. Além disso, o subespaço dos pontos fixos $\left(\mathscr{H}^{0}(\pi ; L)\right)^{G}$ de $G$ sobre $\mathscr{H}^{0}(\pi ; L)$ é isomorfo ao feixe constante $L$ sobre $X / G$. Seja

$$
\mu: \mathscr{H}^{0}(\pi ; L) \longrightarrow\left(\mathscr{H}^{0}(\pi ; L)\right)^{G}
$$

o homomorfismo entre feixes sobre $X / G$, definido por $\mu=\sum_{g \in G} g$. Portanto, o homomorfismo transfer $\tau_{X}$ em (2.3), quando $G$ é finito, será definido como sendo o homomorfismo em cohomologia de feixes de $\mu$,

$$
\tau_{X}: H^{s}(X ; L) \cong H^{s}\left(X / G ; \mathscr{H}^{0}(\pi ; L)\right) \longrightarrow H^{s}(X / G ; L) .
$$

Caso infinito: Suponhamos, primeiramente, que $G$ seja conexo. Neste caso, apresentaremos o homomorfismo transfer construído por Oliver em [50]. 
Seja $\eta_{x}: G \rightarrow G x$ a aplicação equivariante dada por $\eta_{x}(g)=g x$, para todo $g \in G$. Desde que $G$ é conexo, usando (2.2), o homomorfismo induzido

$$
\mathscr{H}^{t}(\pi ; L)_{\breve{x}} \cong H^{t}(G x ; L) \stackrel{\eta_{x}^{*}}{\longrightarrow} H^{t}(G ; L)
$$

é independente de $x$ na sua órbita $\breve{x}$. Consideremos $H^{t}(G ; L)$ como as fibras em $\breve{x}$ do feixe constante $X / G \times H^{t}(G ; L)$. Então, a coleção $\left\{\eta_{x}^{*}\right\}$ determina um homomorfismo de feixes sobre $X / G$

$$
\eta^{*}: \mathscr{H}^{t}(\pi ; L) \longrightarrow H^{t}(G ; L)
$$

Este homomorfismo induz o homomorfismo em cohomologia de feixes

$$
\eta^{*}: H^{s}\left(X / G ; \mathscr{H}^{t}(\pi ; L)\right) \longrightarrow H^{s}\left(X / G ; H^{t}(G ; L)\right)
$$

Seja $\lambda: H^{\operatorname{dim} G}(G ; L) \stackrel{\approx}{\longrightarrow} L$ uma orientação. O homomorfismo transfer $\tau_{X}$ em (2.3), quando $G$ é conexo, será definido pela composição dos seguintes homomorfismos

$$
H^{s+\operatorname{dim} G}(X ; L) \rightarrow E_{\infty}^{s, \operatorname{dim} G} \longmapsto E_{2}^{s, \operatorname{dim} G}=H^{s}\left(X / G ; \mathscr{H}^{\operatorname{dim} G}(\pi ; L)\right),
$$

com o homomorfismo dado em (2.7) e o homomorfismo $\lambda$, para o caso em que $t=\operatorname{dim} G$.

Se $G$ não é necessariamente conexo, seja $G_{0}$ a componente conexa contendo o elemento identidade. Observemos que $G_{0}$ é um subgrupo normal e $G / G_{0}$ é um grupo finito atuando sobre $X / G_{0}$. Para a aplicação órbita

$$
\pi_{0}: X / G_{0} \longrightarrow \frac{X / G_{0}}{G / G_{0}} \approx X / G
$$

temos o homomorfismo transfer no caso finito e, para a aplicação órbita

$$
\pi_{G_{0}}: X \longrightarrow X / G_{0}
$$

temos o homomorfismo transfer no caso conexo. A composição desses dois homomorfismos

$$
H^{s+\operatorname{dim} G}(X ; L) \longrightarrow H^{s}\left(X / G_{0} ; L\right) \longrightarrow H^{s}(X / G ; L)
$$


determina o homomorfismo transfer $\tau_{X}$ em (2.3), quando o grupo $G$ não é conexo. Este homomofismo transfer foi construído de maneira natural, como mostra a seguinte

Proposição 2.2.1. Sejam $G$ um grupo de Lie compacto e $f: X \rightarrow Y$ uma aplicação $G$-equivariante entre $G$-espaços paracompactos. Então, o seguinte diagrama

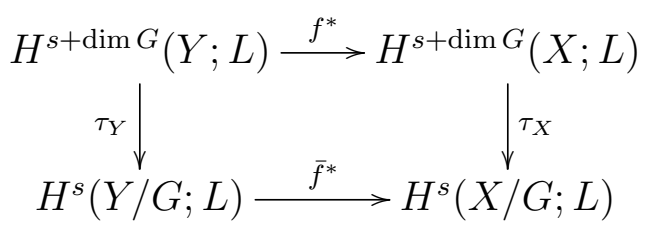

é comutativo, onde $\bar{f}: X / G \rightarrow Y / G$ é a aplicação induzida por $f$ entre os espaços órbita.

\subsection{Prova do teorema principal}

A prova do teorema principal consiste basicamente em demonstrar que o homomofismo transfer é um isomorfismo. Para isso, precisamos provar que o espaço órbita de uma variedade de homologia também é uma variedade de homologia.

DEFINIÇÃo 2.3.1. Uma aplicação própria $f: X \rightarrow Y$ entre espaços de Hausdorff localmente compactos $X$ e $Y$ é $\operatorname{cfs}_{L}^{k}$ (cohomology fiber space) se o feixe de Leray $\mathscr{H}^{i}(f ; L)$ é localmente constante, para todo $i, \operatorname{com} \mathscr{H}^{i}(f ; L)=0$, para $i>k$ e $\mathscr{H}^{k}(f ; L) \neq 0$.

Lema 2.3.2. Suponhamos que $G$ seja um grupo de Lie compacto atuando livremente sobre uma $(L, n)$-variedade de homologia, orientável sobre $L$, onde a $G$-ação preserva a orientação. Se $G$ é conexo e $L$ é um corpo, então $X / G$ é uma $(L, n-\operatorname{dim} G)$-variedade de homologia, orientável sobre $L$.

Demonstração. Se $G$ atua livremente sobre $X$, então a aplicação equivariante $\eta_{x}: G \rightarrow G x$, dada por $\eta_{x}(g)=g x$, para todo $g \in G$, é um homeomorfismo, para qualquer $x \in X$. Portanto, $\operatorname{dim} G \leq n$. Agora, se $G$ é conexo, então o homomorfismo $\eta^{*}$ em (2.6) é um isomorfismo de feixes sobre $X / G$, desde que $\eta_{x}$ é um homeomorfismo. Portanto, os feixes de Leray $\mathscr{H}^{t}(\pi ; L)$ da aplicação órbita são constantes com fibras $H^{t}(G ; L)$. Assim, $\mathscr{H}^{t}(\pi ; L)=0$, para $t>\operatorname{dim} G \mathrm{e}$ $\mathscr{H}^{\operatorname{dim} G}(\pi ; L)=L$, para alguma orientação escolhida $\lambda: H^{n}(G ; L) \stackrel{\approx}{\longrightarrow} L$, ou seja, 
$\pi: X \rightarrow X / G$ é uma $\operatorname{cfs}_{L}^{\operatorname{dim} G}$. Segue de [14, Capítulo V, Teorema 18.5, p. 397], que $X / G$ é uma $(L, n-\operatorname{dim} G)$-variedade de homologia, orientável sobre $L$.

Na Proposição 1.5.1, vimos que se $X$ é uma $(L, n)$-variedade generalizada conexa, então $H_{c}^{n}\left(X ; \mathscr{H}_{n}(X ; L)\right) \cong L$. Esse resultado também é válido para o caso das variedades de homologia no seguinte sentido: se um espaço $Y$ é compacto e conexo, então $H_{c}^{0}(Y ; L) \cong L$. Seja agora $X$ uma $n$-variedade de homologia compacta, conexa e orientável sobre um corpo $L$, então o feixe de orientação sobre $X: \mathscr{H}_{n}(X ; L)$ é isomorfo a $L$ e pela dualidade de Poincaré

$$
H^{n}(X ; L) \cong H_{0}(X ; L) \cong \operatorname{Hom}\left(H_{c}^{0}(X ; L), L\right) \cong L
$$

onde o segundo isomorfismo segue de [5, Teorema 3.3, p. 144].

Proposição 2.3.3. Suponhamos que $G$ seja um grupo de Lie compacto agindo livremente sobre uma $\left(\mathbb{Z}_{p}, n\right)$-variedade de homologia $X, \mathbb{Z}_{p}$-orientável, onde a $G$-ação preserva a orientação. Se $X$ é conexa e compacta, então o homomorfismo transfer

$$
\tau_{X}: H^{n}\left(X ; \mathbb{Z}_{p}\right) \rightarrow H^{n-\operatorname{dim} G}\left(X / G ; \mathbb{Z}_{p}\right)
$$

é um isomorfismo.

Demonstração. Para o caso finito, consideremos a sequência exata curta

$$
0 \longrightarrow \operatorname{ker} \mu \stackrel{\imath}{\longrightarrow} \mathscr{H}^{0}\left(\pi ; \mathbb{Z}_{p}\right) \stackrel{\mu}{\longrightarrow}\left(\mathscr{H}^{0}\left(\pi ; \mathbb{Z}_{p}\right)\right)^{G} \longrightarrow 0
$$

de feixes sobre $X / G$, onde $\mu$ é definido em (2.4) e $\imath$ é a inclusão. A sequência (2.11) induz uma sequência exata em cohomologia

$$
\begin{gathered}
\cdots \longrightarrow H^{n}(X / G ; \operatorname{ker} \mu) \longrightarrow H^{n}\left(X / G ; \mathscr{H}^{0}\left(\pi ; \mathbb{Z}_{p}\right)\right) \stackrel{\tau_{X}}{\longrightarrow} H^{n}\left(X / G ;\left(\mathscr{H}^{0}\left(\pi ; \mathbb{Z}_{p}\right)\right)^{G}\right) \\
\longrightarrow H^{n+1}(X / G ; \operatorname{ker} \mu) \longrightarrow H^{n+1}\left(X / G ; \mathscr{H}^{0}\left(\pi ; \mathbb{Z}_{p}\right)\right) \longrightarrow \cdots
\end{gathered}
$$

Desde que $\operatorname{dim}_{\mathbb{Z}_{p}} X / G=n$, (vide [4, Capítulo III, Proposição 5.1, p. 43]) $H^{n+1}(X / G ; \operatorname{ker} \mu)=0$ e $H^{n}\left(X / G ; \mathbb{Z}_{p}\right) \neq 0$. Consequentemente, esta sequência torna-se

$$
\cdots \longrightarrow H^{n}(X / G ; \operatorname{ker} \mu) \longrightarrow H^{n}\left(X / G ; \mathscr{H}^{0}\left(\pi ; \mathbb{Z}_{p}\right)\right) \stackrel{\tau_{X}}{\longrightarrow} H^{n}\left(X / G ; \mathbb{Z}_{p}\right) \longrightarrow 0
$$


Portanto,

$$
\tau_{X}: H^{n}\left(X ; \mathbb{Z}_{p}\right) \cong H^{n}\left(X / G ; \mathscr{H}^{0}\left(\pi ; \mathbb{Z}_{p}\right)\right) \longrightarrow H^{n}\left(X / G ; \mathbb{Z}_{p}\right) \neq 0
$$

é sobrejetora e, usando o fato em (2.10), concluímos que $\tau_{X}$ é um isomorfismo.

Agora, suponhamos que $G$ seja um grupo conexo. Como na prova do Lema 2.3.2, o homomorfismo $\eta^{*}$ em (2.6) é um isomorfismo. Portanto, o homomorfismo em $(2.7)$

$$
\eta^{*}: H^{s}\left(X / G ; \mathscr{H}^{t}\left(\pi ; \mathbb{Z}_{p}\right)\right) \longrightarrow H^{s}\left(X / G ; H^{t}\left(G ; \mathbb{Z}_{p}\right)\right)
$$

é um isomorfismo, desde que $H^{p}(X / G,-)$ é um funtor. Pelo Lema 2.3.2, $X / G$ é uma $(n-\operatorname{dim} G)$-variedade de homologia, assim, $H^{s}\left(X / G ; \mathscr{H}^{t}\left(\pi ; \mathbb{Z}_{p}\right)\right)=0$, para $s>n-\operatorname{dim} G$. Portanto,

$$
E_{2}^{s, t}=H^{s}\left(X / G ; \mathscr{H}^{t}\left(\pi ; \mathbb{Z}_{p}\right)\right)=0
$$

para $s>n-\operatorname{dim} G$ e para $t>\operatorname{dim} G$. Então, o homomorfismo transfer

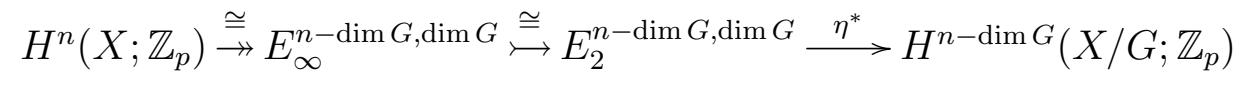

é uma composição de isomorfismos e, portanto, é um isomorfismo.

Se $G$ não é conexo, temos que $\tau_{X}$ é dado pela composição

$$
H^{n}\left(X ; \mathbb{Z}_{p}\right) \longrightarrow H^{n-\operatorname{dim} G}\left(X / G_{0} ; \mathbb{Z}_{p}\right) \longrightarrow H^{n-\operatorname{dim} G}\left(X / G ; \mathbb{Z}_{p}\right)
$$

O fato de que a primeira seta é um isomorfismo segue do caso conexo, previamente demonstrado. Pelo Lema 2.3.2, $X / G_{0}$ é uma $(n-\operatorname{dim} G)$-variedade de homologia compacta, conexa e orientável sobre $\mathbb{Z}_{p}$, e segue do caso finito, que a segunda seta é um isomorfismo, onde $\operatorname{dim} G_{0}=\operatorname{dim} G$.

Observemos que quando $X$ é uma variedade suave, é facil ver, usando a dualidade de Poincaré, que o homomorfismo transfer $\tau_{X}$ é um isomorfismo, desde que ele é o dual de $\pi_{*}: H_{0}\left(X, \mathbb{Z}_{p}\right) \rightarrow H_{0}\left(X / G, \mathbb{Z}_{p}\right)$.

\subsubsection{Prova do teorema principal}

Demonstração. Sejam $q_{X}: X / G \rightarrow B G$ e $q_{Y}: Y / G \rightarrow B G$ as aplicações classificantes para os $G$-fibrados principais $X \rightarrow X / G$ e $Y \rightarrow Y / G$, respectivamente. 
Consideremos o seguinte diagrama

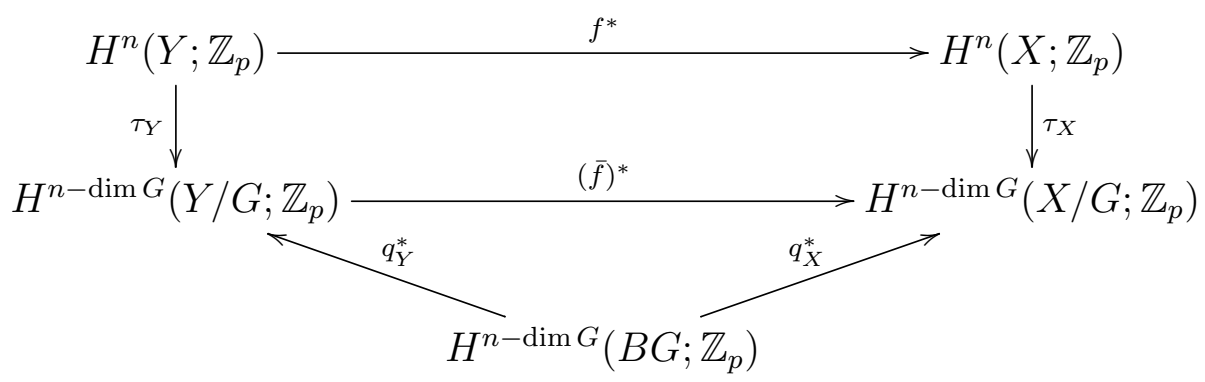

onde $\bar{f}: X / G \rightarrow Y / G$ é a aplicação induzida por $f$ entre os espaços órbita. Pela Proposição 2.2.1, o retângulo no diagrama (2.12) é comutativo. Desde que $q_{X}$ é homotópica a $q_{Y} \circ \bar{f}$, então o triângulo é também comutativo. Além disso, pela Proposição 2.3.3, os homomorfismos transfer $\tau_{X}$ e $\tau_{Y}$ são isomorfismos e, portanto,

$$
\begin{aligned}
& H^{n}\left(X ; \mathbb{Z}_{p}\right) \cong H^{n-\operatorname{dim} G}\left(X / G ; \mathbb{Z}_{p}\right) \cong \mathbb{Z}_{p} \\
& H^{n}\left(Y ; \mathbb{Z}_{p}\right) \cong H^{n-\operatorname{dim} G}\left(Y / G ; \mathbb{Z}_{p}\right) \cong \mathbb{Z}_{p}
\end{aligned}
$$

Sejam $v$ e $u$ classes fundamentais de $H^{n}\left(Y ; \mathbb{Z}_{p}\right)$ e $H^{n}\left(X ; \mathbb{Z}_{p}\right)$, respectivamente. Então, $f^{*}(v)=\operatorname{deg}(f) u$ e segue do diagrama (2.12) que

$$
(\bar{f})^{*}(\bar{v})=\operatorname{deg}(f) \bar{u}
$$

$\operatorname{para} \bar{u}=\tau_{X}(u)$ e $\bar{v}=\tau_{Y}(v)$.

1. Se $q_{X}^{*} \neq 0$, então $(\bar{f})^{*} \neq 0$ e $q_{Y}^{*} \neq 0$. Do diagrama (2.12), concluímos que $f^{*} \neq 0$. Portanto, $f^{*}$ é um isomorfismo.

Agora, seja $b \in H^{n-\operatorname{dim} G}\left(B G ; \mathbb{Z}_{p}\right)$ tal que $q_{X}^{*}(b) \neq 0$ e $q_{Y}^{*}(b) \neq 0$. Então,

$$
\bar{u}=\alpha q_{X}^{*}(b) \quad \bar{v}=\beta q_{Y}^{*}(b)
$$

onde $\alpha, \beta \in \mathbb{Z}$ são não nulos. Logo,

$$
(\bar{f})^{*}(\bar{v})=(\bar{f})^{*}\left(\beta q_{Y}^{*}(b)\right)=\beta q_{X}^{*}(b)
$$

e segue de (2.13), que $\alpha(\operatorname{deg}(f)) \bar{u}=\beta \bar{u}$ e, portanto, $\operatorname{deg}(f) \not \equiv 0(\bmod p)$.

Se $X=Y$, então $u=v, \bar{u}=\bar{v}, \alpha=\beta$ e, portanto, $\operatorname{deg}(f) \equiv 1(\bmod p)$. 
2. Se $q_{X}^{*}=0$ e $q_{Y}^{*} \neq 0$, então $q_{Y}^{*}(b) \neq 0$, para algum $b \in H^{n-\operatorname{dim} G}\left(B G ; \mathbb{Z}_{p}\right)$. Desde que $(\bar{f})^{*}\left(q_{Y}^{*}(b)\right)=0$, então $(\bar{f})^{*}$ não é injetora. Do diagrama (2.12), segue que $f^{*}$ não é injetora e, assim, $f^{*}$ é trivial. Então,

$$
f^{*}(v)=0=(\operatorname{deg}(f)) u
$$

Portanto, $\operatorname{deg}(f) \equiv 0(\bmod p)$. 


\section{Grau de uma aplicação de uma variedade generalizada sobre uma esfera}

\subsection{Introdução}

O primeiro resultado na teoria de grau para aplicações equivariantes foi o famoso teorema de Borsuk-Ulam, o qual afirma que o grau de uma aplicação ímpar de uma esfera finito dimensional em si mesma é ímpar (vide [6] e [7]). Este resultado foi generalizado por S. Eilenberg [24] à aplicações simpliciais comutando com uma ação livre simplicial de um grupo cíclico $\mathbb{Z}_{p}$ ( $p$ primo) sobre esferas. No caso das variedades topológicas e ações não necessariamente livres, foi provado por Alexander Kushkuley e Zatman Balanov [40]. O objetivo deste capitulo é generalizar o método introduzido em [40] para o caso de variedades generalizadas (vide Teorema 3.3.1), encontrando uma relação entre os graus de duas aplicações equivariantes de uma variedade generalizada sobre uma esfera.

Por outro lado, Dold em [22] provou o seguinte teorema:

Se uma aplicação $f: S^{m} \rightarrow S^{n}$ comuta com alguma ação livre de um grupo não trivial finito $G$ sobre as esferas $S^{m}$ e $S^{n}$, então $n \geq m$.

Janey Daccach em seu artigo [20] generalizou este resultado, considerando a seguinte hipótese (H) sobre uma variedade topológica $M^{m}$ com uma $G$-ação livre: Suponha que $S^{m}$ possua uma $G$-ação livre e que exista uma aplicação equivariante $h: M^{m} \rightarrow S^{m}$ tal que o grau de $h$ é relativamente primo à $|G|$.

O resultado principal de J. Daccach é o seguinte:

Teorema. [20, Proposition 2] Sejam $M^{m}$ e $N^{n} G$-variedades livres e suponha $M^{m}$ satisfazendo a hipótese (H). Se $f: M^{m} \rightarrow N^{n}$ é uma aplicação equivariante, então $n \geq m$. 
Utilizando a relação encontrada no Teorema 3.3.1 entre os graus de duas aplicações equivariantes, obtivemos uma generalização do teorema de J. Daccach (vide Teorema 3.4.2). Este resultado será apresentado na última seção do capítulo.

\subsection{Extensão de aplicações equivariantes}

DeFINIÇÃo 3.2.1. Seja $G$ um grupo topológico atuando sobre um espaço métrico $X$. Seja $D_{0} \subset X$ um aberto no seu fecho $\overline{D_{0}}=D$. Então $D$ diz-se um domínio quase-fundamental da $G$-ação sobre $X$ se satisfaz as seguintes condições:

1. $G(D)=X$;

2. $g\left(D_{0}\right) \cap h\left(D_{0}\right)=\emptyset(g \neq h ; g, h \in G)$;

3. $X-G\left(D_{0}\right)=G\left(D-D_{0}\right)$.

Se $X$ possui dimensão finita e se as seguintes condições forem satisfeitas:

4. $\operatorname{dim} D=\operatorname{dim} X / G ; \operatorname{dim}\left(D-D_{0}\right)<\operatorname{dim} D ; \operatorname{dim} G\left(D-D_{0}\right)<\operatorname{dim} X$, então $D$ é chamado domínio fundamental.

Proposição 3.2.2. [40, Teo. 2.1, p. 245]. Para qualquer ação livre de um grupo de Lie compacto sobre um espaço métrico existe um domínio fundamental associado a tal ação.

ObservaÇão 3.2.3. Dado um $G$-espaço métrico $X$ podemos construir duas filtrações de $X$ como descritas a seguir.

1. Se $X$ possui um número finito de tipos de órbitas, sobre o conjunto $\operatorname{Or}(X)$ existe uma escolha de uma indexação admissível

$$
\operatorname{Or}(X)=\left\{\left(H_{1}\right),\left(H_{2}\right), \ldots,\left(H_{m}\right)\right\}
$$

onde $\left(H_{j}\right) \leq\left(H_{i}\right)$ implica que $i<j$. Usando o conjunto indexado $\operatorname{Or}(X)$ podemos definir uma filtração de $X$ da seguinte forma:

$$
X_{1} \subset X_{2} \subset \cdots \subset X_{m}=X
$$

onde

$$
X_{i}=\left\{x \in X \mid\left(G_{x}\right)=\left(H_{j}\right) \text { para algum } j \leq i\right\}
$$

são subespaços $G$-invariantes fechados. Observe que cada $X_{i}$ tem como tipos de órbitas $\left\{\left(H_{1}\right), \ldots,\left(H_{i}\right)\right\}$ e, além disso, $X_{i}-X_{i-1}$ é o fibrado órbita $X_{\left(H_{i}\right)}$. 
2. Seja $A \subset X$ um subespaço $G$-invariante tal que $X-A$ é livre. Sejam

$$
X^{(0)}=X, \quad L^{(0)}=X^{(0)}-A .
$$

Desde que $L^{(0)}$ é um $G$-subespaço livre, segue da Proposição 3.2.2 que existe um domínio fundamental $D^{(0)} \subset L^{(0)}$. Seja $D_{0}^{(0)}$ o aberto em $D^{(0)}=\overline{D_{0}^{(0)}}$ satisfazendo as condições 2-4 na Definição 3.2.1. Com esses termos definamos

$$
X^{(1)}=X^{(0)}-G\left(D_{0}^{(0)}\right)=A \cup G\left(D^{(0)}-D_{0}^{(0)}\right), \quad L^{(1)}=X^{(1)}-A .
$$

Desde que $L^{(1)} \subset L^{(0)}$ é um $G$-subespaço livre, aplicamos novamente a Proposição 3.2 .2 a $L^{(1)}$ obtendo

$$
X^{(2)}=X^{(1)}-G\left(D_{0}^{(1)}\right)=A \cup G\left(D^{(1)}-D_{0}^{(1)}\right), \quad L^{(2)}=X^{(2)}-A,
$$

onde $D_{0}^{(1)}$ é o aberto no domínio fundamental $D^{(1)}=\overline{D_{0}^{(1)}}$ de $L^{(1)}$. Analogamente, obtemos $X^{(3)}$ e $L^{(3)}$ e, assim, sucessivamente. Dessa forma, temos uma filtração de $X$

$$
A \subset \cdots \subset X^{(i)} \subset \cdots \subset X^{(2)} \subset X^{(1)} \subset X^{(0)}=X
$$

onde cada $X^{(i)}$ é um subespaço $G$-invariante fechado de $X$, dado por

$$
X^{(i)}=A \cup G\left(D^{(i-1)}-D_{0}^{(i-1)}\right), \quad L^{(i)}=X^{(i)}-A,
$$

sendo $D_{0}^{(i-1)}$ o aberto no domínio fundamental $D^{(i-1)}=\overline{D_{0}^{(i-1)}}$ em $L^{(i-1)}$. Pela condição (4) na Definição 3.2.1, temos que

$$
\operatorname{dim} G\left(D^{(0)}-D_{0}^{(0)}\right)<\operatorname{dim} L^{(0)}=\operatorname{dim}(X-A) .
$$

Analogamente,

$$
\operatorname{dim} G\left(D^{(1)}-D_{0}^{(1)}\right)<\operatorname{dim} L^{(1)}=\operatorname{dim} G\left(D^{(0)}-D_{0}^{(0)}\right)<\operatorname{dim}(X-A),
$$


e, assim, sucessivamente, temos

$$
\operatorname{dim} G\left(D^{(i)}-D_{0}^{(i)}\right)<\cdots<\operatorname{dim} G\left(D^{(0)}-D_{0}^{(0)}\right)<\operatorname{dim}(X-A)
$$

Portanto, se $\operatorname{dim}(X-A)$ é finita, então a filtração (3.2) é finita, ou seja, existe um $l$ tal que $X^{(l)}=A$.

Lema 3.2.4. [23, Prop. 8.1.5]. Sejam $\left\{X_{i}\right\}_{i=1, \ldots, m}$ e $\left\{Y_{i}\right\}_{i=1, \ldots, m}$ as filtrações dos $G$-espaços métricos $X$ e $Y$, respectivamente. Suponha que $s: X_{i}^{H_{i}} \rightarrow Y_{i}^{H_{i}}$ seja uma extensão $W\left(H_{i}\right)$-equivariante da aplicação $W\left(H_{i}\right)$-equivariante $\left.\delta\right|_{X_{i-1}^{H_{i}}}$, onde $\delta: X_{i-1} \rightarrow Y_{i-1}$ é uma aplicação $G$-equivariante. Então, existe uma única extensão G-equivariante $\gamma: X_{i} \rightarrow Y_{i}$ de $\delta$ tal que $\left.\gamma\right|_{X_{i} H_{i}}=s$.

Proposição 3.2.5. [40, Prop. 3.1, p. 248]. Suponhamos que um grupo de Lie $G$ atue sobre um espaço métrico $X$ e seja $A$ um subespaço fechado $G$-invariante de $X$, tal que a ação de $G$ sobre $X-A$ é livre. Se $X-A$ é finito dimensional, então qualquer aplicação $G$-equivariante $A \rightarrow Y$ se estende sobre $X$ se, para todo $i \geq 1$, qualquer aplicação $G$-equivariante $X^{(i)} \rightarrow Y$ tem uma extensão (não necessariamente equivariante) sobre $X^{(i)} \cup D^{(i-1)}$.

Proposição 3.2.6. [40, Teo. 3.1, p. 249] Sejam $X$ e $Y$ G-espaços métri$\cos$ e $A \subset X$ um subconjunto fechado $G$-invariante. Suponha que $G$ seja um grupo de Lie compacto atuando sobre $X-A$ com um número finito de tipos de órbitas $\left(H_{1}\right),\left(H_{2}\right), \ldots,\left(H_{m}\right)$. Suponha, além disso, que o espaço $Y^{H_{i}}$ seja localmente e globalmente $k$-conexo, para cada $k=0,1, \ldots, n_{i}(i=1, \ldots, m)$. Se $\operatorname{dim}\left(X_{\left(H_{i}\right)}-A\right) / G \leq n_{i}+1$, para todo $i$, então qualquer aplicação equivariante $f: A \rightarrow Y$ tem uma extensão $G$-equivariante sobre $X$.

\subsection{Teorema principal}

Sejam $X$ uma $(\mathbb{Z}, n)$-variedade generalizada e $S^{n}$ uma $n$-esfera. Desde que $S^{n}$ é uma $(\mathbb{Z}, n)$-variedade generalizada orientável e o funtor imagem inversa de um feixe preserva feixes constantes, então toda aplicação contínua $f: X \rightarrow S^{n}$ é orientável se, e somente se, $X$ é orientável. Além disso, se $X$ for segundo enumerável, então pelo Teorema de Metrização de Urysohn, $X$ é metrizável e, portanto, paracompacto. Nesse caso, $\operatorname{dim}_{\mathbb{Z}} X=\operatorname{dim} X$ (vide Observação 1.1.2). 
Teorema 3.3.1. Sejam $X$ uma variedade generalizada $n$-dimensional sobre $\mathbb{Z}$ segundo enumerável, compacta, conexa e orientada e $S=S^{n}$ a esfera $n$-dimensional. Seja $G$ um grupo finito atuando sobre $X$ e $S$ com $G$-ações concordantes. Seja $A \subset X$ um subconjunto fechado $G$-invariante. Sejam $\left(H_{1}\right),\left(H_{2}\right), \ldots,\left(H_{m}\right)$ os tipos de órbitas em $X-A$. Suponha que o conjunto $S^{H_{i}}$ seja localmente e globalmente $k$-conexo, para todo $k=0,1, \ldots, \operatorname{dim} X^{H_{i}}-1$, onde $i=1, \ldots, m$. Então, para todo par de aplicações $G$-equivariantes $\phi, \psi: X \rightarrow S$ que são $G$ equivariantemente homotópicas sobre $A$, temos

$$
\operatorname{deg}(\psi) \equiv \operatorname{deg}(\phi) \quad\left(\bmod \operatorname{mdc}\left\{\left|G / H_{1}\right|, \ldots,\left|G / H_{m}\right|\right\}\right)
$$

Demonstração. Se $\operatorname{deg}(\psi)=\operatorname{deg}(\phi)$, então não temos nada a demonstrar. Vamos supor que $\operatorname{deg}(\psi) \neq \operatorname{deg}(\phi)$ e que, além disso, são não nulos. Portanto, pela Proposição 1.6.5, $\psi$ e $\phi$ são sobrejetoras.

Definamos a $G$-ação cilíndrica sobre o cilindro $C=X \times[0,1]$ da seguinte forma. Para todo $g \in G,(x, t) \in X \times I$,

$$
(g,(x, t)) \mapsto(g x, t)
$$

Considere a $(n+1)$-bola fechada $B$ com bordo $S$. Desde que o cone $C(S)=$ $(S \times[0,1]) /(S \times\{0\})$ com vértice $y_{0}$ é homeomorfo a $B$, onde $y_{0}$ é identificado com o centro $O$ da bola $B$, então podemos definir sobre $B$ uma $G$-ação cônica da seguinte forma: para todo $g \in G$ e $[(y, t)] \in C(S) \approx B$,

$$
g[(y, t)]= \begin{cases}{[(g y, t)]} & \text { se } t \in(0,1] \\ y_{0} & \text { se } t=0\end{cases}
$$

Assim, definidas estas ações, temos que $C^{H}=X^{H} \times I$ e $C(S)^{H}=C\left(S^{H}\right)$, para qualquer subgrupo $H$ de $G$.

Consideremos os subespaços $G$-invariantes

$$
N=X \times\{0,1\} \bigcup A \times[0,1] \text { e } B^{*}=B-O
$$

de $C$ e $B$, respectivamente. Usando as aplicações $G$-equivariantes $\phi, \psi: X \rightarrow S$ que são homotopicamente $G$-equivariantes sobre $A$, podemos definir a seguinte 
aplicação $G$-equivariante sobrejetora $f_{0}: N \rightarrow B^{*}$ dada por:

$$
f_{0}(x, t)= \begin{cases}\phi(x) & \text { se } x \in X, t=0, \\ {[(F(x, t), t)]} & \text { se }(x, t) \in A \times(0,1), \\ \psi(x) & \text { se } x \in X, t=1,\end{cases}
$$

onde $F: A \times I \rightarrow S$ é uma homotopia $G$-equivariante entre $\left.\phi\right|_{A}$ e $\left.\psi\right|_{A}$.

Nosso objetivo é encontrar uma extensão $G$-equivariante $f: C \rightarrow B$ de $f_{0}$, satisfazendo as seguintes condições:

$(\alpha) K=f^{-1}(O)=\bigcup_{j=1}^{m} T_{j}, T_{s} \cap T_{p}=\emptyset$, se $s \neq p ;$

( $\beta) T_{j}=G\left(K_{j}\right)$ para algum subconjunto compacto $K_{j}$ de $C$;

$(\gamma) H_{j}\left(K_{j}\right)=K_{j}$

( $\delta) g\left(K_{j}\right) \cap h\left(K_{j}\right)=\emptyset$ se $g h^{-1} \notin H_{j}(j=1, \ldots, m)$.

Para construir $f$, usaremos indução sobre os tipos de órbitas de $X-A$. Para isso, consideremos a filtração de $X-A$, como na Observação 3.2.3-1

$$
X_{1} \subset X_{2} \subset \cdots \subset X_{i} \subset \cdots \subset X_{m}=X-A
$$

onde cada subespaço fechado $G$-invariante $X_{i}$ é dado por

$$
X_{i}=\left\{x \in X-A \mid\left(G_{x}\right)=\left(H_{j}\right) \text { para algum } j \leq i\right\}
$$

Esta filtração induz uma filtração sobre $C-A \times I$

$$
C_{1} \subset C_{2} \subset \cdots \subset C_{i} \subset \cdots \subset C_{m}=C-A \times I
$$

onde $C_{i}=X_{i} \times I$. Desde que $G_{(x, t)}=G_{x}$, para todo $(x, t) \in C$, segue que $C_{i}=\left\{(x, t) \in C-A \times I \mid\left(G_{(x, t)}\right)=\left(H_{j}\right)\right.$, para algum $\left.j \leq i\right\}$ e, dessa forma, cada $C_{i}$ possui $i$ tipos de órbitas, ou seja, $\left\{\left(H_{1}\right),\left(H_{2}\right), \ldots,\left(H_{i}\right)\right\}$. Agora, consideremos a filtração $\left\{B_{i}^{*}\right\}$ do espaço $B^{*}$ por subconjuntos fechados. Denotaremos

$$
B_{i}=B_{i}^{*} \cup\{O\}
$$

Portanto, o processo indutivo sobre os tipos de órbitas consiste em estender 
$f_{0}$ a uma aplicação $G$-equivariante

$$
f_{i}: C_{i} \cup N \rightarrow B
$$

satisfazendo as condições $(\alpha),(\beta),(\gamma)$ e $(\delta)$, substituindo $m$ (número de tipos de órbitas em $C_{m}$ ) por $i$ (número de tipos de órbitas em $C_{i}$ ).

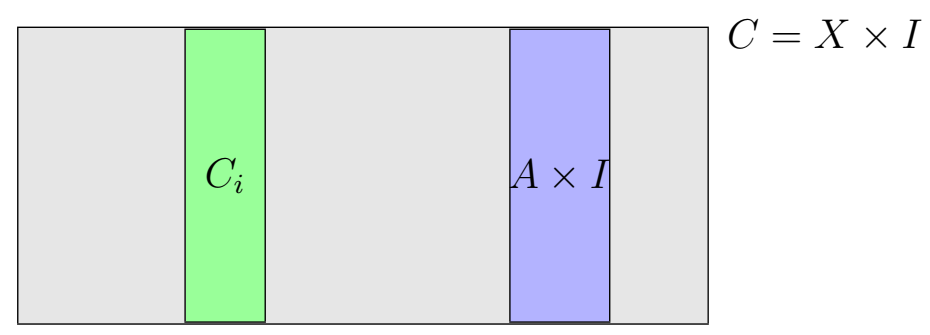

Para encontrar $f_{i}$, basta encontrar uma extensão $G$-equivariante $\tilde{f}_{i}: C_{i} \rightarrow B_{i}$ de $\left.f_{0}\right|_{C_{i} \cap \partial C}$, satisfazendo as condições descritas acima. Neste caso, $f_{i}$ será definida como

$$
f_{i}(x)= \begin{cases}\tilde{f}_{i}(x), & \text { se } x \in C_{i} \\ f_{0}(x), & \text { se } x \in N\end{cases}
$$

Para $i=1$ : Por abuso de notação, denotemos $H=H_{1}$. Como foi mencionado acima, $C_{1}$ possui um tipo de órbita, ou seja, $(H)$. O conjunto dos pontos fixos $C_{1}^{H}$ é um $W(H)$-espaço (vide Observação 1.7.2). Seja $(x, t) \in C_{1}^{H}$. O subgrupo de isotropia

$$
W(H)_{(x, t)}=\{n H \in W(H) \mid(n H)(x, t)=n(x, t)=(x, t)\}
$$

é igual a $H$ (identidade do grupo $W(H))$ se, e somente se, $G_{(x, t)}=H$. Desde que $G_{(x, t)}=G_{x}=H$, para todo $(x, t) \in C_{1}$, segue que, para todo $(x, t) \in C_{1}^{H}$, $W(H)_{(x, t)}=H$, ou seja, $C_{1}^{H}$ é um $W(H)$-subespaço livre. Em particular, o conjunto $C_{1}^{H}-\left(C_{1} \cap \partial C\right)^{H}$ é livre. Então, pela Observação 3.2.3(2), existe uma filtração

$$
\left(C_{1} \cap \partial C\right)^{H} \subset \cdots \subset C_{1}^{(j)} \subset \cdots \subset C_{1}^{(2)} \subset C_{1}^{(1)} \subset C_{1}^{(0)}=C_{1}^{H}
$$

onde cada subespaço $W(H)$-invariante $C_{1}^{(j)}$ é dado por

$$
C_{1}^{(j)}=\left(C_{1} \cap \partial C\right)^{H} \cup G\left(D^{(j-1)}-D_{0}^{(j-1)}\right), \quad L^{(j)}=C_{1}^{(j)}-\left(C_{1} \cap \partial C\right)^{H},
$$


sendo $D_{0}^{(j-1)}$ o aberto no domínio fundamental $D^{(j-1)}=\overline{D_{0}^{(j-1)}}$ em $L^{(j-1)}$. Pela mesma Observação 3.2.3(2), existe um $l \in \mathbb{N}$, tal que $C^{(l)}=\left(C_{1} \cap \partial C\right)^{H}$, desde que $\operatorname{dim}\left(C_{1}^{H}-\left(C_{1} \cap \partial C\right)^{H}\right)<\infty$. Da hipótese sobre $S$, segue que $B_{1}^{*}$ é localmente e globalmente $k$-conexo, para cada $k=0,1, \ldots, s=\operatorname{dim} X^{H}-1$. Portanto, pela Proposição 3.2.6, existe uma extensão $W(H)$-equivariante $f^{(1)}: C_{1}^{(1)} \rightarrow\left(B_{1}^{*}\right)^{H}$ de $\left.f_{0}\right|_{(\partial C)^{H}}$. Logo, desde que

$$
C_{1}^{(1)}=\left(C_{1} \cap \partial C\right)^{H} \cup W(H)\left(D^{(0)}-D_{0}^{(0)}\right)=C_{1}^{H}-W(H)\left(D_{0}^{(0)}\right),
$$

podemos estender ainda mais $\left.f_{0}\right|_{(\partial C)^{H}}$ à aplicação $W(H)$-equivariante

$$
s: C_{1}^{H} \rightarrow B_{1}^{H},
$$

$s^{-1}(O)=\{$ união disjunta de $|W(H)|$ conjuntos compactos os quais são permutados por $W(H)\}$.

Pelo Lema 3.2.4 existe uma aplicação $G$-equivariante $\tilde{f}_{1}: C_{1} \rightarrow B_{1}$ que estende $\left.f_{0}\right|_{C_{1} \cap \partial C}$

Para $i>1$ : Neste caso, suponhamos a seguinte hipótese de indução.

Hipótese de indução: Seja $\tilde{f}_{i}: C_{i} \rightarrow B_{i}$ uma aplicação $G$-equivariante com $i \in\{0,1, \cdots, m-1\}$ fixo tal que $\left.\tilde{f}_{i}\right|_{\left(C_{i} \cap \partial C\right)}=f_{0} \mathrm{e}$

( $\alpha)\left(\tilde{f}_{i}\right)^{-1}(O)=\bigcup_{j=1}^{i} T_{j}^{i}$, onde $T_{s}^{i} \cap T_{p}^{i}=\emptyset$ se $s \neq p ;$

( $\beta) T_{j}^{i}=G\left(K_{j}^{i}\right)$, para algum subconjunto compacto $K_{j}^{i}$ de $C_{i}$;

$(\gamma) H_{j}\left(K_{j}^{i}\right)=K_{j}^{i}$;

( $\delta) g\left(K_{j}^{i}\right) \cap h\left(K_{j}^{i}\right)=\emptyset$, se $g h^{-1} \notin H_{j}$.

Devemos construir uma extensão $G$-equivariante $\tilde{f}_{i+1}: C_{i+1} \rightarrow B_{i+1}$ de $\tilde{f}_{i}$ com as propriedades $(\alpha)-(\delta)$ (substuindo $i+1$ em vez de $i$ ).

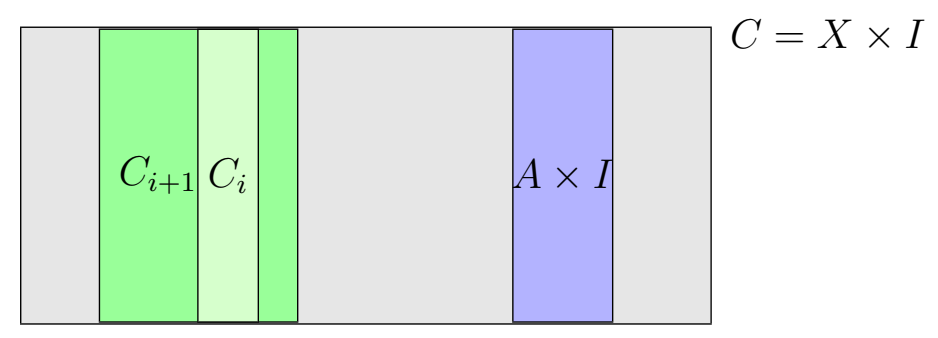


Começamos considerando a aplicação $G$-equivariante

$$
\ell: C_{i} \cup\left(\partial C \cap C_{i+1}\right) \rightarrow B_{i+1}
$$

dada por

$$
\ell(x)= \begin{cases}\tilde{f}_{i}(x), & \text { se } x \in C_{i} \\ f_{0}(x), & \text { se } x \in \partial C \cap C_{i+1}\end{cases}
$$

Seja

$$
V_{i+1}=C_{i}^{H_{i+1}} \cup\left(\partial C \cap C_{i+1}^{H_{i}}\right) \subset C_{i+1}^{H_{i+1}}
$$

A restrição

$$
\left.\ell\right|_{V_{i+1}}: V_{i+1} \rightarrow B_{i+1}^{H_{i+1}}
$$

é $W\left(H_{i+1}\right)$-equivariante, que estende a aplicação $W\left(H_{i+1}\right)$-equivariante

$$
\left(\tilde{f}_{i}\right)^{H_{i+1}}: C_{i}^{H_{i+1}} \rightarrow B_{i}^{H_{i+1}}
$$

Pela hipótese de indução, seja $U\left(K_{j}^{i}\right)$ uma vizinhança suficientemente pequena $H_{j}$-invariante $(j \leq i)$. Então,

1. $U\left(T_{j}^{i}\right)=G\left[U\left(K_{j}^{i}\right)\right]=\bigcup_{g \in G} g U\left(K_{j}^{i}\right)=\bigcup_{s=1}^{\left|G / H_{j}\right|} g_{s} U\left(K_{j}^{i}\right)$, onde $\left\{g_{s}\right\}_{s=1}^{\left|G / H_{j}\right|}$ são os representantes de $G / H_{j}$, é uma vizinhança $G$-invariante de $T_{j}^{i}$;

2. $g_{l} U\left(K_{j}^{i}\right) \cap g_{r} U\left(K_{j}^{i}\right)=\emptyset$, se $l \neq r$;

3. $U\left(K_{t}^{i}\right) \cap U\left(K_{p}^{i}\right)=\emptyset$, se $t \neq p$.

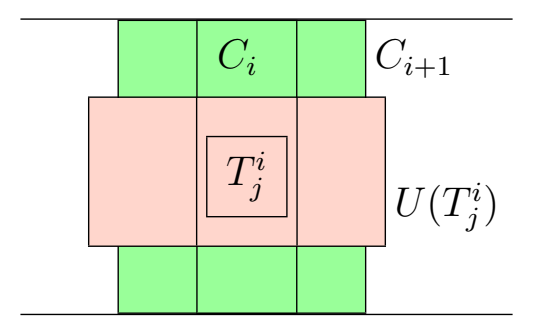

Seja $\tilde{U}\left(T_{j}^{i}\right)=U\left(T_{j}^{i}\right) \cap C_{i+1}^{H_{i+1}}$ e, portanto, $\tilde{U}\left(T_{j}^{i}\right)$ é $W\left(H_{i+1}\right)$-invariante. Então, existe uma vizinhança fechada $W\left(H_{i+1}\right)$-invariante $V\left(T_{j}^{i}\right)$ de $T_{j}^{i}$ contida em $\tilde{U}\left(T_{j}^{i}\right)$ tal que $\operatorname{dim} \partial V\left(T_{j}^{i}\right)<\operatorname{dim} C_{i+1}^{H_{i+1}}$ (vide [25, Teo. 7.3.11]). Seja

$$
U^{(i)}=\bigcup_{j=1}^{i} V\left(T_{j}^{i}\right)
$$


Então,

$$
\begin{gathered}
W\left(H_{i+1}\right)\left(U^{(i)}\right)=U^{(i)} \\
\operatorname{dim}\left(\partial U^{(i)}\right)<\operatorname{dim} C_{i+1}^{H_{i+1}}=\operatorname{dim} X^{H_{i+1}}+1 .
\end{gathered}
$$

A última desigualdade segue da Proposição 1.1.3-(4). Usando (3.3) e o fato que $W\left(H_{i+1}\right)$ atua livremente sobre $C_{i+1}^{H_{i+1}}-C_{i}^{H_{i+1}}$, concluímos que $W\left(H_{i+1}\right)$ atua livremente sobre

$$
\partial U^{(i)}-\left(\partial U^{(i)} \cap V_{i+1}\right) \subset C_{i+1}^{H_{i+1}}
$$

O conjunto $\left(B_{i+1}^{*}\right)^{H_{i+1}}$ é localmente e globalmente $k$-conexo, para cada $k=$ $0,1, \ldots, \operatorname{dim} X^{H_{i+1}}-1$. Em particular, de $(3.4),\left(B_{i+1}^{*}\right)^{H_{i+1}}$ é localmente e globalmente $k$-conexo para cada $k=0,1, \ldots, \operatorname{dim} \partial U^{(i)}-1$. Desde que

$$
V_{i+1} \cup \partial U^{(i)}-V_{i+1}=\partial U^{(i)}-\left(\partial U^{(i)} \cap V_{i+1}\right)
$$

é livre e $\operatorname{dim}\left(V_{i+1} \cup \partial U^{(i)}-V_{i+1}\right) / G \leq \operatorname{dim} \partial U^{(i)}$, temos pela Proposição 3.2 .6 que existe uma extensão $W\left(H_{i+1}\right)$-equivariante

$$
\hat{\ell}: V_{i+1} \cup \partial U^{(i)} \rightarrow B_{i+1}^{H_{i+1}}
$$

da aplicação $\left.\ell\right|_{V_{i+1}}$. Desde que a diferença de $V_{i+1} \cup U^{(i)}$ e $V_{i+1} \cup \partial U^{(i)}$ é livre, aplicamos novamente a Proposição 3.2.6 e, encontramos uma extensão $W\left(H_{i+1}\right)$ equivariante $s_{i}: V_{i+1} \cup U^{(i)} \rightarrow\left(B_{i+1}^{*}\right)^{H_{i+1}}$ de $\hat{\ell}$. Finalmente, o conjunto

$$
C^{H_{i+1}}-\left(V_{i+1} \cup U^{(i)}\right)
$$

é um $W\left(H_{i+1}\right)$-subespaço livre do $W\left(H_{i+1}\right)$-espaço $C_{i+1}^{H_{i+1}}$. Pela Observação 3.2.3, existe uma filtração por subconjuntos fechados $W\left(H_{i+1}\right)$-invariantes

$$
V_{i+1} \cup U^{(i)} \subset \cdots \subset C_{i+1}^{(2)} \subset C_{i+1}^{(1)} \subset C_{i+1}^{(0)}=C_{i+1}^{H_{i+1}}
$$

do espaço $C_{i+1}^{H_{i+1}}$, tal que

$$
\begin{aligned}
\operatorname{dim}\left(C_{i+1}^{(0)}-C_{i+1}^{(r)}\right) & <\operatorname{dim}\left(C_{i+1}^{(0)}-C_{i+1}^{(r+1)}\right) \\
& \leq \operatorname{dim}\left((X-A)^{H_{i+1}}\right)+1 \\
& =\operatorname{dim}\left(X_{\left(H_{i+1}\right)}\right)+1 \quad(r=0,1, \ldots, l-1) .
\end{aligned}
$$


Desde que $V_{i+1} \cup U^{(i)} \cup C_{i+1}^{(1)}-\left(V_{i+1} \cup U^{(i)}\right)$ é livre, segue da Proposição 3.2.6, que $s_{i}$ possui uma extensão $W\left(H_{i+1}\right)$-equivariante

$$
f_{i}^{\prime}: V_{i+1} \cup U^{(i)} \cup C_{i+1}^{(1)} \longrightarrow B_{i+1}^{H_{i+1}}
$$

de tal maneira que $\left(f_{i}^{\prime}\right)^{-1}(O) \subset U^{(i)}$. Além disso, pela Proposição 3.2.5, existe uma aplicação continua (não necessariamente equivariante)

$$
f_{i}^{\prime \prime}: V_{i+1} \cup U^{(i)} \cup C_{i+1}^{(1)} \cup D_{i+1}^{(0)} \longrightarrow B_{i+1}^{H_{i+1}}
$$

de $f_{i}^{\prime}$ (aquí, $D_{i+1}^{(0)}$ denota o domínio fundamental para o $W\left(H_{i+1}\right)$-espaço livre $\left.C^{H_{i+1}}-\left(V_{i+1} \cup U^{(i)} \cup C_{i+1}^{(1)}\right)\right)$. Denote por $L_{i+1}$ o conjunto

$$
L_{i+1}=\left(\left.f_{i}^{\prime \prime}\right|_{D_{i+1}^{(0)}}\right)^{-1}(O) .
$$

Pelo Teorema [13, Teo. 3.3, p. 39], a aplicação $\left.f^{\prime \prime}\right|_{D_{i+1}^{(0)}}: D_{i+1}^{(0)} \rightarrow B_{i+1}^{H_{i+1}}$ possui uma extensão $W\left(H_{i+1}\right)$-equivariante $\hat{s}: W\left(H_{i+1}\right) D_{i+1}^{(0)} \rightarrow B_{i+1}^{H_{i+1}}$, onde

$$
W\left(H_{i+1}\right) D_{i+1}^{(0)}=C_{i+1}^{H_{i+1}}-\left(V_{i+1} \cup U^{(i)} \cup C_{i+1}^{(1)}\right),
$$

ou seja, existe uma aplicação $W\left(H_{i+1}\right)$-equivariante

$$
\hat{s}: C_{i+1}^{H_{i+1}}-\left(V_{i+1} \cup U^{(i)} \cup C_{i+1}^{(1)}\right) \longrightarrow B_{i+1}^{H_{i+1}} .
$$

Definimos $s: C_{i+1}^{H_{i+1}} \rightarrow B_{i+1}^{H_{i+1}}$ dada por

$$
s(x)= \begin{cases}\hat{s}(c), & c \in C_{i+1}^{H_{i+1}}-\left(V_{i+1} \cup U^{(i)} \cup C_{i+1}^{(1)}\right), \\ f_{i}^{\prime}(c), & c \in V_{i+1} \cup U^{(i)} \cup C_{i+1}^{(1)}\end{cases}
$$

Portanto, s é uma extensão $W\left(H_{i+1}\right)$-equivariante de $\left(\tilde{f}_{i}\right)^{H_{i+1}}: C_{i}^{H_{i+1}} \rightarrow B_{i}^{H_{i+1}}$.

Pelo Lema 3.2.4 existe uma aplicação $G$-equivariante $\tilde{f}_{i+1}: C_{i+1} \rightarrow B_{i+1}$ que estende $\tilde{f}_{i}$ tal que $\left.\tilde{f}_{i+1}\right|_{C_{i+1}^{H_{i+1}}}=s$. Além disso, denotemos por

$$
K_{i+1}^{i+1}=L_{i+1}, \quad K_{j}^{i+1}=K_{j}^{i} \cup\left(\tilde{f}_{i+1}^{-1}(O)\right) \cap U\left(K_{j}^{i}\right), \quad j=1,2, \ldots, i+1,
$$

e observemos que 
1. $H_{j}\left(K_{i+1}^{i+1}\right)=H_{j}\left(L_{i+1}\right)=H_{j}\left(\left(\left.f_{i}^{\prime \prime}\right|_{D_{i+1}^{(0)}}\right)^{-1}(O)\right)=\left(\left.f_{i}^{\prime \prime}\right|_{D_{i+1}^{(0)}}\right)^{-1}(O)=K_{i+1}^{i+1}$.

2. $H_{j}\left(K_{j}^{i+1}\right)=H_{j}\left(K_{j}^{i} \cup\left(\tilde{f}_{i+1}^{-1}(O)\right) \cap U\left(K_{j}^{i}\right)\right)=K_{j}^{i} \cup\left(\tilde{f}_{i+1}^{-1}(O)\right) \cap U\left(K_{j}^{i}\right)$.

3. Para $s \neq p, G\left(K_{s}^{i+1}\right) \cap G\left(K_{p}^{i+1}\right)=$

$$
=\left(T_{s}^{i} \cup\left(\tilde{f}_{i+1}^{-1}(O)\right) \cap G\left(U\left(K_{s}^{i}\right)\right)\right) \cap\left(T_{p}^{i} \cup\left(\tilde{f}_{i+1}^{-1}(O)\right) \cap G\left(U\left(K_{p}^{i}\right)\right)\right)=\emptyset .
$$

4. Se $g h^{-1} \in H_{j}$, então $g K_{j}^{i} \cap h K_{j}^{i}=\emptyset$,

$$
\begin{aligned}
& g\left(\tilde{f}_{i+1}^{-1}(O)\right) \cap g U\left(K_{j}^{i}\right) \cap h K_{j}^{i}=\emptyset, \\
& g K_{j}^{i} \cap h\left(\tilde{f}_{i+1}^{-1}(O)\right) \cap h U\left(K_{j}^{i}\right)=\emptyset \mathrm{e} \\
& g\left(\tilde{f}_{i+1}^{-1}(O)\right) \cap g U\left(K_{j}^{i}\right) \cap h\left(\tilde{f}_{i+1}^{-1}(O)\right) \cap h U\left(K_{j}^{i}\right)=\emptyset . \text { Logo, } \\
& g K_{j}^{i+1} \cap h K_{j}^{i+1}=\emptyset .
\end{aligned}
$$

Portanto, existe uma extensão $G$-equivariante $\tilde{f}_{i+1}: C_{i+1} \rightarrow B_{i+1}$ de $\tilde{f}_{i}$ tal que

( $\alpha)\left(\tilde{f}_{i+1}\right)^{-1}(O)=\bigcup_{j=1}^{i+1} T_{j}^{i+1}$, onde $T_{s}^{i+1} \cap T_{p}^{i+1}=\emptyset$, se $s \neq p$.

(ß) $T_{j}^{i+1}=G\left(K_{j}^{i+1}\right)$, para algum subconjunto compacto $K_{j}^{i+1}$ de $C_{i+1}$.

$(\gamma) H_{j}\left(K_{j}^{i+1}\right)=K_{j}^{i+1}$.

( $\delta) g\left(K_{j}^{i+1}\right) \cap h\left(K_{j}^{i+1}\right)=\emptyset$, se $g h^{-1} \notin H_{j}$.

Assim, concluímos que existe uma extensão $G$-equivariante $f: C \rightarrow B$ da aplicação $f_{0}$ satisfazendo as seguintes condições:

( $\alpha) K=f^{-1}(O)=\bigcup_{j=1}^{m} T_{j}, T_{s} \cap T_{p}=\emptyset$ se $s \neq p ;$

( $\beta) T_{j}=G\left(K_{j}\right)$, para algum compacto $K_{j}$;

$(\gamma) H_{j}\left(K_{j}\right)=K_{j}$

( $\delta) g\left(K_{j}\right) \cap h\left(K_{j}\right)=\emptyset$, se $g h^{-1} \in H_{j}(j=1, \cdots, m)$.

Podemos ver $f$ como uma aplicação de pares entre variedades generalizadas com bordo $(C, \partial C) \rightarrow(B, S)$. Assim, o grau de $f$, coincide com o número inteiro $d=\operatorname{deg}(f)$ tal que

$$
f_{*}\left(O_{K}\right)=d . O_{o},
$$

onde $O_{K} \in H_{n}(C, C-K), O_{o} \in H_{n}(B, B-O), f_{*}: H_{n}(C, C-K) \rightarrow H_{n}(B, B-O)$ e $K=f^{-1}(O)$. Logo,

$$
\operatorname{deg}(f)=\operatorname{deg}\left(\left.f\right|_{\partial C}\right)=\operatorname{deg}\left(\left.f\right|_{X \sqcup X}\right)= \pm(\operatorname{deg}(\psi)-\operatorname{deg}(\phi))
$$


Pela condição $(\alpha)$, temos

$$
\operatorname{deg}(f)=\sum_{j=1}^{m} \operatorname{deg}\left(f_{j}\right)
$$

onde $f_{j}$ é uma restrição de $f$ a vizinhanças suficientemente pequenas de $T_{j}$. De acordo com as condições $(\beta)-(\delta)$, todo $T_{j}$ pode ser representado como uma união de conjuntos disjuntos $K_{j_{s}}$, ou seja, $T_{j}=G\left(K_{j}\right)=\bigcup_{g \in G} g K_{j}=\bigcup_{i=1}^{\left|G / H_{j}\right|} K_{j_{s}}$ tal que cada $K_{j_{s}}$ é uma translação de $K_{j}$. Portanto, pela Proposição 1.6.10

$$
\operatorname{deg}\left(f_{j}\right)=\sum_{s=1}^{\left|G / H_{j}\right|} \operatorname{deg}\left(f_{j_{s}}\right),
$$

onde $f_{j_{s}}$ é a restrição de $f_{j}$ a uma vizinhança suficientemente pequena de $K_{j_{s}}$. Agora, usando propriedades de grau, temos que $\operatorname{deg}\left(f_{j_{s}}\right)$ são todos iguais a $a_{j}$, desde que cada $K_{j_{s}}\left(s=1,2, \cdots,\left|G / H_{j}\right|\right)$ é uma translação de $K_{j}$, o grau não se altera. Assim, $\operatorname{deg}\left(f_{j}\right)=a_{j} \cdot\left|G / H_{j}\right|$, e usando (3.6) e (3.7) segue que

$$
\operatorname{deg}(f)=\sum_{j=1}^{m} a_{j}\left|G / H_{j}\right| .
$$

Portanto, de (3.5) temos $\operatorname{deg}(\psi) \equiv \operatorname{deg}(\phi)\left(\bmod m d c\left\{\left|G / H_{1}\right|, \cdots,\left|G / H_{m}\right|\right\}\right)$.

\subsection{Existência de aplicações equivariantes}

DeFinIÇÃo 3.4.1. Seja $G$ um grupo finito não trivial atuando sobre uma $(\mathbb{Z}, n)$ variedade generalizada $X$ compacta, conexa e orientada com tipos de órbitas $\left(H_{1}\right), \ldots,\left(H_{l}\right)$. Dizemos que $X$ satisfaz a propriedade $(\Gamma)$ se existe uma ação de $G$ sobre a $n$-esfera $S$ tal que:

1. O conjunto $S^{H_{i}}$ é localmente e globalmente $k$-conexo para todo $k=0,1, \cdots, n_{i}$, onde $i=1, \ldots, l$ e $\operatorname{dim} X_{\left(H_{i}\right)} \leq n_{i}+1$;

2. Existe uma aplicação $G$-equivariante $\psi: X \rightarrow S$ para a qual deg $\psi$ é relativamente primo a $\operatorname{mcd}\left\{\left|G / H_{i}\right|\right\}_{i=1}^{l}=\alpha(G)$.

Teorema 3.4.2. Seja $X$ uma variedade generalizada $n$-dimensional sobre $\mathbb{Z}$ segundo enumerável, compacta, conexa e orientada com a propriedade $(\Gamma)$ e $\alpha(G)>1$. Suponhamos que $Y$ seja um $G$-espaço métrico, $\left(D_{1}\right), \cdots,\left(D_{r}\right)$ são 
os tipos em órbitas em $Y$, e os conjuntos $S^{D_{j}}$ sejam localmente e globalmente $s$-conexos, para todo $s=0,1, \ldots, n_{j}$, com $j=1, \ldots, r$, e $\operatorname{dim} Y_{\left(D_{j}\right)} \leq n_{j}+1$. Se existe uma aplicação equivariante $f: X \rightarrow Y$, então $n \leq \operatorname{dim} Y$.

Demonstração. Seja $A=\emptyset \subset Y$, assim $\left\{\left(D_{1}\right),\left(D_{2}\right), \ldots,\left(D_{r}\right)\right\}$ são os tipos de órbitas de $Y-\emptyset=Y$. Pelas condições sobre $S^{D_{j}}$, segue da Proposição 3.2.6 que existe uma aplicação $G$-equivariante $\psi: Y \rightarrow S$. Seja $f: X \rightarrow Y$ uma aplicação $G$-equivariante e mostremos que $H^{n}(Y ; \mathbb{Z}) \neq 0$. Consequentemente, $n \leq \operatorname{dim}_{\mathbb{Z}} Y=\operatorname{dim} Y$. Suponhamos que $H^{n}(Y ; \mathbb{Z})=0$ e considere a aplicação $G$ equivariante $\psi \circ f: X \rightarrow S$, deste modo, $\operatorname{deg}(\psi \circ f)=0$. Pela Propriedade $(\Gamma)(2)$, existe uma aplicação $G$-equivariante $\phi: X \rightarrow S$ tal que $\operatorname{deg}(\phi)$ é relativamente primo a $\alpha(G)$. Pela Propriedade $(\Gamma)(1)$, segue do Teorema 3.3.1 que

$$
\operatorname{deg}(\psi \circ f) \equiv \operatorname{deg}(\phi) \quad(\bmod \alpha(G))
$$

ou seja, $\operatorname{deg}(\psi \circ f)-\operatorname{deg}(\phi)=q \cdot \alpha(G)$, para algum $q \in \mathbb{Z}$. Então, segue que

$$
\operatorname{deg}(\phi)=q \cdot \alpha(G)
$$

o que contradiz o fato que $\operatorname{deg}(\phi)$ é relativamente primo a $\alpha(G)$.

Observação 3.4.3. Na Definição 3.4.1, a hipótese $(\Gamma)$ para uma $(\mathbb{Z}, n)$-variedade generalizada $X$ com uma $G$-ação livre se reduz a hipótese $(H)$ no teorema principal de J. Daccach em [20], ou seja, neste caso $X$ satisfaz a hipótese $(\Gamma)$ se existe uma ação de $G$ sobre a n-esfera $S$ tal que existe uma aplicação $G$-equivariante $\psi: X \rightarrow S$ para a qual deg $\psi$ é relativamente primo a $|G|$.

Como uma consequência do Teorema 3.4.2, considerando o caso de $G$-ações livres, obtivemos a seguinte generalização do resultado de Daccach.

Corolário 3.4.4. Sejam X e $Y G$-variedades generalizadas livres sobre $\mathbb{Z}$, compactas, conexas de dimensões $n$ e $m$ respectivamente e, suponha $X$ satisfazendo a hipótese $(\Gamma)$. Se $f: X \rightarrow Y$ é uma aplicação equivariante, então $m \geq n$. 


\section{A}

\section{Cohomologia e co-cohomologia de feixes}

Neste capítulo apresentaremos de modo sucinto a teoria de feixes, cohomologia de feixes e a homologia de Borel-Moore. A construção dos módulos de (co)homologia (absoluto e relativo) e seus respectivos homomorfismos induzidos são apresentados nas Seções 2 e 3. Na Seção 1 abordaremos os conceitos básicos da teoria de feixes e família de suportes. Este apêndice não pretende ser uma exposição completa desta teoria, para detalhes recomendamos a leitura do livro [14, Cap. I, II e V] que apresenta uma visão da Topologia Algébrica do ponto de vista da teoria de feixes. Outras referências para o estudo desta teoria são [30, Cap. II] e [58].

\section{A.1. Feixes de módulos e Família de suportes}

Durante todo este capítulo, $L$ denotará um anel comutativo com unidade 1 e a palavra módulo significará um $L$-módulo.

A.1.1. Feixes de módulos. Seja $X$ um espaço topológico.

Definição A.1.1. Um feixe (de módulos) sobre $X$ é um par $\mathscr{A}=(\mathscr{A}, \pi)$, onde $\mathscr{A}$ é um espaço topológico (em geral, não satisfaz a condição de ser Hausdorff) e $\pi: \mathscr{A} \rightarrow X$ é um homeomorfismo local tais que:

1. Para cada $x \in X$, o conjunto $\mathscr{A}_{x}=\pi^{-1}(x)$, é um módulo, chamado a fibra de $\mathscr{A}$ em $x$ (stalk).

2. As operações de módulo são contínuas no seguinte sentido: Seja $\mathscr{A} \Delta \mathscr{A}$ o subespaço $\{(\alpha, \beta) \in \mathscr{A} \times \mathscr{A} \mid \pi(\alpha)=\pi(\beta)\}$. Então, as operações de módulo $\mathscr{A} \Delta \mathscr{A} \rightarrow \mathscr{A},(\alpha, \beta) \mapsto \alpha-\beta$ e $L \times \mathscr{A} \rightarrow \mathscr{A},(l, \alpha) \mapsto l \alpha$ são contínuas. 
Exemplo A.1.2. Seja $M$ um módulo. O espaço produto $X \times M$ (munindo $M$ com a topologia discreta) com a primeira projeção $\pi_{X}: X \times M \rightarrow X$ determinam um feixe. Desde que suas fibras são isomorfas a $M$, em geral usaremos a notação $M=\left(X \times M, \pi_{X}\right)$.

Definição A.1.3. Seja $\mathscr{A}=(\mathscr{A}, \pi)$ um feixe sobre $X$.

1. Subfeixe: Um subfeixe $\mathscr{B}$ de $\mathscr{A}$ é um subespaço aberto de $\mathscr{A}$ tal que, para todo $x \in X, \mathscr{B}_{x}=\mathscr{B} \cap \mathscr{A}_{x}$ é um submódulo de $\mathscr{A}_{x}$.

2. Feixe restrição: Seja $A$ um subespaço de $X$. O subespaço $\pi^{-1}(A)$ junto com a aplicação $\left.\pi\right|_{\pi^{-1}(A)}$ formam um feixe sobre $A$, chamado a restrição de $\mathscr{A}$ a $A$ e denotado por $\left.\mathscr{A}\right|_{A}$. Além disso, para todo $x \in A,\left(\left.\mathscr{A}\right|_{A}\right)_{x}=\mathscr{A}_{x}$.

3. Feixe imagem inversa: Seja $f: D \rightarrow X$ uma aplicação contínua. A imagem inversa de $\mathscr{A}$ por $f$ é o feixe $f^{*} \mathscr{A}=\left(f^{*} \mathscr{A}, \bar{\pi}\right)$ sobre o domínio $D$ definido por

$$
f^{*} \mathscr{A}:=\{(d, a) \in D \times \mathscr{A} \mid f(d)=\pi(a)\}
$$

e $\bar{\pi}: f^{*} \mathscr{A} \rightarrow D$ dado por $\bar{\pi}(d, a)=d$. A estrutura de módulo sobre $\left(f^{*} \mathscr{A}\right)_{d}$ é dado pelo isomorfismo $f_{d}^{*}: \mathscr{A}_{f(d)} \rightarrow\left(f^{*} \mathscr{A}\right)_{d}, a \mapsto(d, a)$, para todo $d \in D$.

4. Seção: Seja $A$ um subespaço de $X$. Uma seção de $\mathscr{A}$ sobre $A$ é uma aplicação contínua $s: A \rightarrow \mathscr{A}$ tal que $\pi \circ s$ é a inclusão $\imath: A \rightarrow X$, ou seja, $s(x) \in \mathscr{A}_{x}$, para todo $x \in X$. Se $A=X$, uma seção $s: X \rightarrow \mathscr{A}$ será chamada uma seção global. Denotaremos por $\mathscr{A}(A)$ o conjunto de todas as seções sobre $A$. Em particular, $\mathscr{A}(X)$ denotará o conjunto de todas as seções globais.

ObservaÇão A.1.4. Seja $\mathscr{A}=(\mathscr{A}, \pi)$ um feixe sobre $X$. Desde que $\pi$ é um homeomorfismo local, a coleção de todas as imagens de seções sobre subconjuntos abertos de $X$ é uma base para a topologia de $\mathscr{A}$ (vide [58, p. 19]).

Definição A.1.5. Sejam $\mathscr{A}$ e $\mathscr{B}$ feixes sobre $X$. Um homomorfismo de feixes $h: \mathscr{A} \rightarrow \mathscr{B}$ é uma aplicação contínua tal que $h\left(\mathscr{A}_{x}\right) \subset \mathscr{B}_{x}$ para todo $x \in X$, e as restrições $h_{x}: \mathscr{A}_{x} \rightarrow \mathscr{B}_{x}$ de $h$ às fibras são homomorfismos de módulos.

A coleção de todos os feixes sobre $X$ junto com os homomorfismo de feixes formam a categoria $\mathcal{S} h(X)$. A coleção de todos os homomorfismo de feixes $\mathscr{A} \rightarrow \mathscr{B}$ será denotada por $\operatorname{Hom}(\mathscr{A}, \mathscr{B})$. Se o funtor contravariante $\operatorname{Hom}(-, \mathscr{I})$ é exato, dizemos que $\mathscr{I}$ é um feixe injetivo. Seja $h: \mathscr{A} \rightarrow \mathscr{B}$ um homomorfismo de feixes sobre $X$. Segundo a definição categórica de isomorfismo, $h$ é um isomorfismo se 
$h$ é um homeomorfismo e, para todo $x \in X$, os homomorfismos $h_{x}$ são isomorfismos. Neste caso, denotaremos $\mathscr{A} \simeq \mathscr{B}$. O kernel ker $h=\{s \in \mathscr{A} \mid h(s)=0\}$ e a imagem $\operatorname{Im} h=\{h(s) \in \mathscr{B} \mid s \in \mathscr{A}\}$ de $h$ são subfeixes de $\mathscr{A}$ e $\mathscr{B}$, respectivamente. A aplicação $h$ é um monomorfismo (respectivamente, um epimorfismo) se ker $h=\{0\}$ (respectivamente, $\operatorname{Im} h=\mathscr{B}$ ). Portanto, desde que $h$ é um aplicação aberta (segue da Observação A.1.4), monomorfismo e epimorfismo implicam isomorfismo.

Definição A.1.6. Seja $M$ um módulo. Um feixe $\mathscr{A}$ sobre $X$, diz-se constante com fibras $M$, se $\mathscr{A} \simeq X \times M$. Em particular, $X \times M$ é um feixe constante com fibras $M$. Um feixe $\mathscr{A}$ sobre $X$ diz-se localmente constante com fibras $M$, se todo ponto de $X$ tem uma vizinhança $U$ tal que $\left.\mathscr{A}\right|_{U}$ é constante com fibras $M$.

Sequências exatas também são definidas naturalmente como segue: uma sequência $\cdots \rightarrow \mathscr{A} \stackrel{h}{\longrightarrow} \mathscr{B} \stackrel{k}{\longrightarrow} \mathscr{C} \rightarrow \cdots$ de homomorfismos de feixes é exata em $\mathscr{B}$ se ker $k=\operatorname{Im} h$. Se for exata em cada feixe, dizemos simplesmente que a sequência é exata.

Definição A.1.7. Um feixe graduado $\mathscr{L}^{*}$ é uma sequência $\left\{\mathscr{L}^{p}\right\}$ de feixes, $p$ variando sobre os inteiros. Um feixe diferencial é um feixe graduado junto com homomorfismos de feixes $d: \mathscr{L}^{p} \rightarrow \mathscr{L}^{p+1}$ tal que $d^{2}: \mathscr{L}^{p} \rightarrow \mathscr{L}^{p+2}$ é zero, para todo $p$. Uma resolução de um feixe $\mathscr{A}$ é um feixe diferencial $\mathscr{L}^{*}$ com $\mathscr{L}^{p}=0$, para $p<0$, junto com um homomorfismo chamado aumento $\epsilon: \mathscr{A} \rightarrow \mathscr{L}^{0}$ tal que a sequência $0 \longrightarrow \mathscr{A} \stackrel{\epsilon}{\longrightarrow} \mathscr{L}^{0} \stackrel{d}{\longrightarrow} \mathscr{L}^{1} \stackrel{d}{\longrightarrow} \mathscr{L}^{2} \longrightarrow \cdots$ é exata. Uma aplicação $f: \mathscr{L}^{*} \rightarrow \mathscr{K}^{*}$ de resoluções de um feixe $\mathscr{A}$ é uma coleção de homomorfismos $\left\{f^{p}: \mathscr{L}^{p} \rightarrow \mathscr{K}^{p}\right\}_{p \in \mathbb{N}}$ tal que o seguinte diagrama comuta

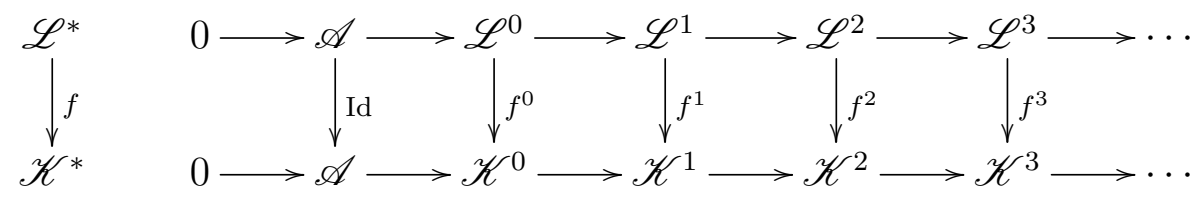

Nosso objetivo agora é descrever um feixe como um funtor contravariante com certas condições. Para isso começaremos definindo um prefeixe.

DeFinição A.1.8. Um prefeixe (de módulos) A sobre $X$ é uma correspondência que associa, a cada subconjunto aberto $U$ de $X$ um módulo $A(U)$ e a cada par $U \subset V$ de subconjuntos abertos de $X$, associa um homomorfismo de módulos $r_{U, V}: A(V) \rightarrow A(U)$ (chamado restrição) satisfazendo as seguintes condições: 
1. $r_{U, U}=\operatorname{Id}_{A(U)}$, (identidade)

2. $r_{U, W}=r_{U, V} \circ r_{V, W}$ quando $U \subset V \subset W$.

Exemplo A.1.9. Seja $M$ um módulo. O prefeixe constante $M_{X}$ é definido para todo subconjunto aberto $U$ de $X$ por $M_{X}(U)=M$, com restrições $r_{U, V}=\operatorname{Id}_{M}$ a identidade sobre $M$, para todo $U \subset V$.

Observemos que um prefeixe $A$ sobre $X$ é um funtor contravariante da categoria dos subconjuntos abertos de $X$ e inclusões na categoria dos módulos e homomorfismos de módulos. Assim, um morfismo entre prefeixes é simplesmente uma transformação natural. Mais especificamente, temos a seguinte.

Definição A.1.10. Sejam $A$ e $B$ prefeixes sobre $X$. Um homomorfismo de prefeixes $h: A \rightarrow B$ é uma coleção de homomorfismos de módulos $h_{U}: A(U) \rightarrow$ $B(U)$ tal que para todo $U \subset V$,

$$
r_{U, V}^{B} \circ h_{V}=h_{U} \circ r_{U, V}^{A},
$$

onde $r_{U, V}^{B}: B(V) \rightarrow B(U)$ e $r_{U, V}^{A}: A(V) \rightarrow A(U)$ são as restrições de $\mathscr{A}$ e $\mathscr{B}$, respectivamente.

Dado um feixe podemos definir um prefeixe e reciprocamente. Após definir esses termos veremos que um feixe pode ser visto como um prefeixe com certas condições (Observação A.1.12).

(P) Prefeixe de seções: Sejam $\mathscr{A}$ um feixe sobre $X$ e $A$ um subespaço de $X$. Podemos ver que o conjunto $\mathscr{A}(A)$ possui estrutura de módulo e a restrição $\mathscr{A}(A) \rightarrow \mathscr{A}\left(A^{\prime}\right),\left.s \mapsto s\right|_{A^{\prime}}$, para $A^{\prime} \subset A$, forma um homomorfismo de módulos. Em particular, para subconjuntos abertos $U$ de $X$ a correspondência $U \mapsto$ $\mathscr{A}(U)$ define um prefeixe sobre $X$ chamado prefeixe de seções de $\mathscr{A}$.

(S) Feixe gerado por um prefeixe: Seja $A$ um prefeixe sobre $X$. Considere a soma topológica

$$
E=\bigcup_{U \subset X} U \times A(U)
$$

onde $U$ é munido da topologia de subespaço e $A(U)$ é munido da topologia discreta. Sobre esse conjunto, definimos a seguinte relação de equivalência: se $(x, s) \in U \times A(U)$ e $(y, t) \in V \times A(V)$, então $(x, s) \sim(y, t)$ se, e somente se, $x=$ $y$ e existe uma vizinhança aberta $W$ de $x \operatorname{com} W \subset U \cap V$ e $r_{W, U}(s)=r_{W, V}(t)$. 
Sejam $\mathscr{A}=E / \sim$ o espaço quociente e $\pi: \mathscr{A} \rightarrow X$, a aplicação induzida pela aplicação $p: E \rightarrow X, p(x, s)=x$, ou seja, $\pi \circ q=p$ onde $q: E \rightarrow \mathscr{A}$ é a projeção canônica. Podemos ver que o conjunto $\pi^{-1}(x)$ é da forma:

$$
\mathscr{A}_{x}=\pi^{-1}(x) \cong \lim _{\longrightarrow} A(U)
$$

onde $U$ varia ao longo das vizinhanças abertas de $x$ em $X$. O conjunto $\mathscr{A}_{x}$ herda uma estrutura de módulo canônico dos grupos $A(U)$. Denotamos por $s_{x}$ os elementos $[x, s]$ de $\mathscr{A}_{x}$. Portanto, pela topologia quociente em $\mathscr{A}$, segue que o par $(\mathscr{A}, \pi)$ define um feixe sobre $X$, chamado o feixe gerado pelo prefeixe $A$ e denotado por

$$
\mathscr{A}=\operatorname{Sheaf}(A) \quad \text { ou } \quad \mathscr{A}=\text { Sheaf }(U \mapsto A(U)) .
$$

Exemplo A.1.11. O feixe gerado pelo prefeixe constante $M_{X}$ é o feixe constante $X \times M$, para todo módulo $M$.

Observação A.1.12. Seja $\mathscr{A}_{0}$ um feixe sobre $X$ e $A$ o prefeixe de seções de $\mathscr{A}_{0}$. Pela Observação A.1.4 o homomorfismo de feixes $\mathscr{A}_{0} \rightarrow \mathscr{A}=\operatorname{Sheaf}(A)$, dado por $s(x) \mapsto s_{x}$, é um isomorfismo. Reciprocamente, seja $A$ um prefeixe sobre $X$ satisfazendo a seguinte propriedade: dada uma cobertura aberta $\left\{U_{\alpha}\right\}$ de um subconjunto aberto $U \subset X$, a sequência

$$
0 \longrightarrow A(U) \stackrel{f}{\longrightarrow} \prod_{\alpha} A\left(U_{\alpha}\right) \stackrel{g}{\longrightarrow} \prod_{\langle\alpha, \beta\rangle} A\left(U_{\alpha} \cap U_{\beta}\right)
$$

é exata, onde

$$
f:=\prod_{\alpha} r_{U_{\alpha}, U} \quad \text { e } \quad g:=\prod_{\langle\alpha, \beta\rangle}\left(r_{U_{\alpha} \cap U_{\beta}, U_{\alpha}}-r_{U_{\beta} \cap U_{\beta}, U_{\alpha}}\right) .
$$

Agora, seja $\mathscr{A}=$ Sheaf $(A)$. Então, os homomorfismos $\theta_{U}: A(U) \rightarrow \mathscr{A}(U)$ dados por $\theta_{U}(s)(x)=s_{x}$, para $s \in A(U)$ e $x \in U$ formam um isomorfismo de prefeixes. Portanto, os prefeixes satisfazendo a condição (A.2) coincidem com a noção de feixe. Para maiores detalhes desta observação vide [14, Cap. I, p. 6].

\section{DefinIÇÃo A.1.13.}

1. Feixe quociente: Sejam $\mathscr{A}$ um feixe sobre $X$ e $\mathscr{B}$ um subfeixe de $\mathscr{A}$. Temos que $\mathscr{B}(A) \subset \mathscr{A}(A)$, para todo subespaço $A$ de $X$. O feixe quociente $\mathscr{A} / \mathscr{B}$ é 
definido como

$$
\mathscr{A} / \mathscr{B}:=\text { Sheaf }(U \mapsto \mathscr{A}(U) / \mathscr{B}(U)) \text {. }
$$

2. Feixe soma direta, produto tensorial e produto torção: Sejam $\mathscr{A}$ e $\mathscr{B}$ feixes sobre $X$. A soma direta $\mathscr{A} \oplus \mathscr{B}$, o produto tensorial $\mathscr{A} \otimes \mathscr{B}$ e o produto torção $\mathscr{A} * \mathscr{B}$ são os feixes definidos por

$$
\mathscr{A} \# \mathscr{B}=\operatorname{Sheaf}(U \mapsto \mathscr{A}(U) \# \mathscr{B}(U))
$$

onde \# denota as operações algébricas $\oplus, \otimes \mathrm{e} *$, respectivamente. Além disso, segue do isomorfismo (A.1) que $(\mathscr{A} \# \mathscr{B})_{x} \cong \mathscr{A}_{x} \# \mathscr{B}_{x}$, para todo $x \in X$.

A.1.2. Família de suportes. Seja $X$ um espaço topológico.

DeFinição A.1.14. Uma família de suportes sobre $X$ é uma família $\Phi$ de subconjuntos fechados de $X$ tais que:

1. Um subconjunto fechado de um elemento de $\Phi$ é um elemento de $\Phi$.

2. A união finita de elementos de $\Phi$ é ainda um elemento de $\Phi$.

Dizemos que a família de suportes $\Phi$ é paracompactificante se, em adição:

3. Cada elemento de $\Phi$ é paracompacto.

4. Cada elemento de $\Phi$ tem uma vizinhança fechada contida em $\Phi$.

\section{EXemplo A.1.15.}

1. Todos os subconjuntos compactos de $X$ formam uma família de suportes, chamada família de suporte compacto de $X$, e denotada por $\boldsymbol{c}$. Esta família de suportes é paracompactificante quando $X$ for localmente compacto.

2. Todos os subconjuntos fechados de $X$ também formam uma família de suportes, chamada família de suporte fechado. É habitual "denotar" esta família pela ausência de um símbolo. Em alguns casos, denotaremos por $\boldsymbol{c l d}$. Esta família de suportes é paracompactificante quando $X$ for paracompacto.

3. Se $A \subset X$ e $\Phi$ é uma família de suportes sobre $X$, então $\Phi \cap A$ denota a família $\{K \cap A \mid K \in \Phi\}$ de suportes sobre $A$, e $\left.\Phi\right|_{A}$ denota a família $\{K \mid K \subset$ $A$ e $K \in \Phi\}$ de suportes sobre $A$ ou $X$.

4. Se $f: X \rightarrow Y$ é uma aplicação, então $f^{-1} \Psi$ denota a família sobre $X$ de todos os subconjuntos fechados de conjuntos da forma $f^{-1} K$, para $K \in \Psi$. 
Sejam $\mathscr{A}$ um feixe sobre $X$ e $s: X \rightarrow \mathscr{A}$ uma seção global. Note que o conjunto $\{x \in X, s(x) \neq 0\}$ é fechado, desde que seu complementar é aberto (segue da Observação A.1.4). Portanto, temos a seguinte

Definição A.1.16. Sejam $\mathscr{A}$ um feixe sobre $X$ e $s: X \rightarrow \mathscr{A}$ uma seção global. Definimos o suporte de uma seção $s \in \mathscr{A}(X)$ como sendo o conjunto fechado $|s|=\{x \in X \mid s(x) \neq 0\}$. Além disso, o conjunto

$$
\Gamma_{\Phi}(\mathscr{A}):=\{s \in \mathscr{A}(X)|| s \mid \in \Phi\}
$$

é um submódulo de $\mathscr{A}(X)$, chamado módulo das seções com suporte em $\Phi$.

Até aqui, temos obtido quatro funtores covariantes importantes:

(1) Para uma aplicação contínua $f: X \rightarrow Y$ temos o funtor $f^{*}: \operatorname{Sh}(Y) \rightarrow \mathcal{S} h(X)$, $\mathscr{B} \mapsto f^{*} \mathscr{B}$, associando a cada homomorfismo de feixes $h: \mathscr{B}_{1} \rightarrow \mathscr{B}_{2}$, o homomorfismo de feixes $f^{*} h: f^{*} \mathscr{B}_{1} \rightarrow f^{*} \mathscr{B}_{2}$ dado por $(x, b) \mapsto(x, h(b))$.

(2) O funtor $\operatorname{Sh}(X) \rightarrow \mathcal{P S h}(X), \mathscr{A} \mapsto(U \mapsto \mathscr{A}(U))$, associando a cada homomorfismo de feixes $h: \mathscr{A} \rightarrow \mathscr{B}$, o homomorfismo de prefeixes de seções $h_{U}: \mathscr{A}(U) \rightarrow \mathscr{B}(U)$ dado por $s \mapsto h \circ s$.

(3) O funtor Sheaf $: \mathcal{P S h}(X) \rightarrow \mathcal{S} h(X), A \rightarrow$ Sheaf $(A)$, associando a cada homomorfismo de prefeixes $h: A \rightarrow B$, o homomorfismo de feixes gerados $h: \mathscr{A} \rightarrow \mathscr{B}$, o qual é induzido pelo homomorfismo de módulos $h_{x}: \mathscr{A}_{x}=$ $\lim A(U) \rightarrow \mathscr{B}_{x}=\lim B(U)$, ao longo das vizinhanças abertas $U$ de $x$ (vide isomorfismo (A.1)).

(4) Para uma família de suportes $\Phi$ sobre $X$, temos o funtor $\Gamma_{\Phi}: \mathcal{S} \hbar(X) \rightarrow \operatorname{Mod}$, $\mathscr{A} \mapsto \Gamma_{\Phi}(\mathscr{A})$ associando a cada homomorfismo de feixes $\alpha: \mathscr{A} \rightarrow \mathscr{B}$, o homomorfismo de módulos $\Gamma_{\Phi}(\alpha): \Gamma_{\Phi}(\mathscr{A}) \rightarrow \Gamma_{\Phi}(\mathscr{B})$ dado por $s \mapsto \alpha \circ s$.

ObservaÇão A.1.17. Os funtores (1) e (3) são exatos e (2) e (4) são exatos à esquerda $^{1}$ (vide [14, p. 9, 13 e 22]). Pela exatidão do funtor Sheaf, a sequência exata curta de prefeixes $0 \rightarrow \mathscr{B}(U) \rightarrow \mathscr{A}(U) \rightarrow \mathscr{A}(U) / \mathscr{B}(U) \rightarrow 0$, para todo subfeixe $\mathscr{B}$ de $\mathscr{A}$, induz a sequência exata curta

$$
0 \longrightarrow \mathscr{B} \longrightarrow \mathscr{A} \longrightarrow \mathscr{A} / \mathscr{B} \longrightarrow 0
$$

\footnotetext{
${ }^{1}$ Vide [36, Cap. II].
} 


\section{A.2. Cohomologia de feixes com suporte}

A.2.1. Módulos de cohomologia. Seja $\mathscr{A}=(\mathscr{A}, \pi)$ um feixe sobre um espaço topológico $X$. Para cada subconjunto aberto $U$ de $X$, denotamos por $C^{0}(U ; \mathscr{A})$ a coleção de todas as funções (não necessariamente contínuas) $f: U \rightarrow \mathscr{A}$ tais que $\pi \circ f=\operatorname{Id}_{U}$. A correspondência

$$
U \mapsto C^{0}(U ; \mathscr{A})=\prod_{x \in U} \mathscr{A}_{x}
$$

define um prefeixe sobre $X$, desde que $C^{0}(U ; \mathscr{A})$ é um módulo e as restrições $r_{U, V}: C^{0}(V ; \mathscr{A}) \rightarrow C^{0}(U ; \mathscr{A})$ dadas por $\left.f \rightarrow f\right|_{U}$ para $U \subset V$ são homomorfismos de módulos. Este prefeixe satisfaz a condição (A.2), portanto, de acordo com a Observação A.1.12 esse prefeixe é um feixe, que denotaremos por $\mathscr{C}^{0}(X ; \mathscr{A})$. Desde que o funtor Sheaf é exato, a inclusão de prefeixes $\mathscr{A}(U) \hookrightarrow C^{0}(U ; \mathscr{A})=$ $\mathscr{C}^{0}(X ; \mathscr{A})(U)$, induz um monomorfismo de feixes

$$
\epsilon: \mathscr{A} \rightarrow \mathscr{C}^{0}(X ; \mathscr{A})
$$

Denotemos por $\mathscr{Z}^{0}(X ; \mathscr{A})$ ao feixe $\mathscr{A}$. Então, por indução podemos definir, para qualquer $p \geq 1$,

$$
\begin{aligned}
& \mathscr{C}^{p}(X ; \mathscr{A}):=\mathscr{C}^{0}\left(X ; \mathscr{Z}^{p}(X ; \mathscr{A})\right), \\
& \mathscr{Z}^{p}(X ; \mathscr{A}):=\mathscr{C}^{p-1}(X ; \mathscr{A}) / \mathscr{Z}^{p-1}(X ; \mathscr{A}) .
\end{aligned}
$$

Pelo monomorfismo (A.4), $\mathscr{Z}^{p}(X ; \mathscr{A})$ é um subfeixe de $\mathscr{C}^{p}(X ; \mathscr{A})$ e segue da Observação A.1.17 que existem as sequências exatas curtas (setas pretas):

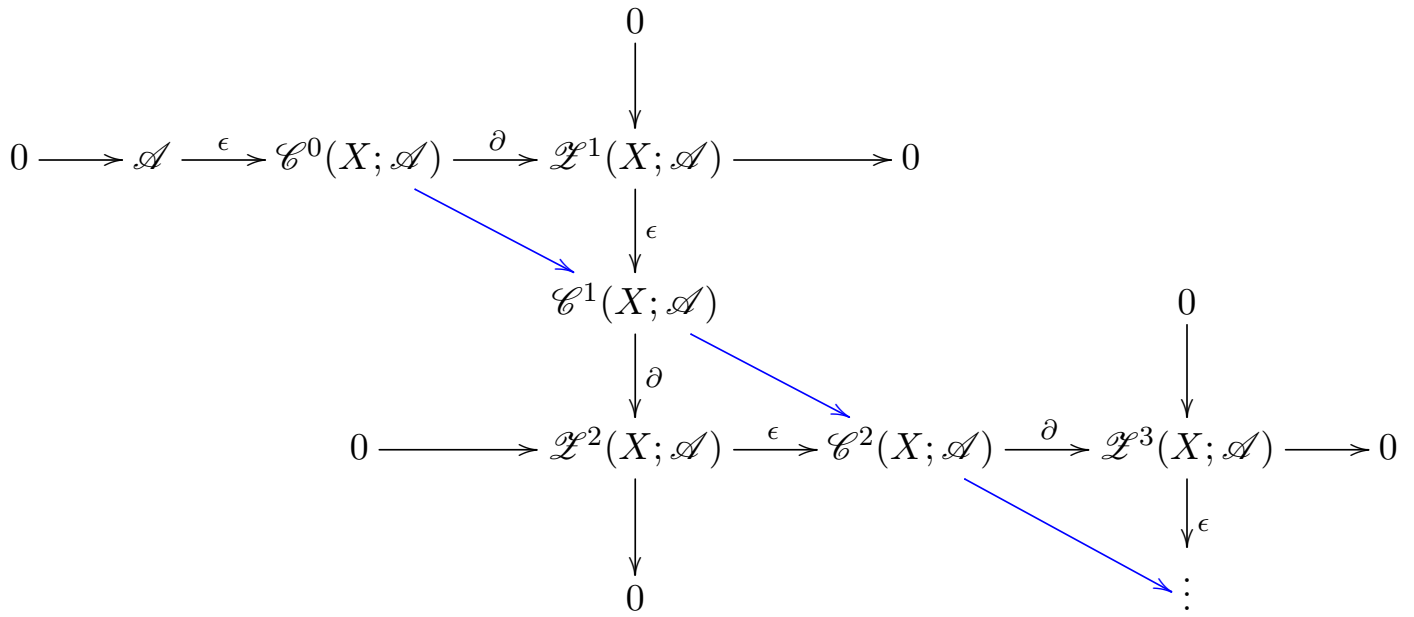


Considerando $d=\epsilon \circ \partial$, temos a seguinte sequência exata (setas azuis)

$$
0 \longrightarrow \mathscr{A} \stackrel{\epsilon}{\longrightarrow} \mathscr{C}^{0}(X ; \mathscr{A}) \stackrel{d}{\longrightarrow} \mathscr{C}^{1}(X ; \mathscr{A}) \stackrel{d}{\longrightarrow} \mathscr{C}^{2}(X ; \mathscr{A}) \stackrel{d}{\longrightarrow} \cdots
$$

Obtemos assim uma resolução de $\mathscr{A}$, denotada por $\mathscr{C}^{*}(X ; \mathscr{A})$ e devida a R. Godement.

Agora, seja $\Phi$ uma família de suportes sobre $X$. Aplicando o funtor $\Gamma_{\Phi}$ a $\mathscr{C}^{*}(X ; \mathscr{A})$, obtemos o complexo de cocadeias

$$
C_{\Phi}^{*}(X ; \mathscr{A}):=\Gamma_{\Phi}\left(\mathscr{C}^{*}(X ; \mathscr{A})\right)
$$

Definição A.2.1. Para uma família $\Phi$ de suportes sobre $X$ e para um feixe $\mathscr{A}$ sobre $X$, definimos o p-ésimo módulo de cohomologia de $X$, com coeficientes em $\mathscr{A}$ e suporte em $\Phi$ por

$$
H_{\Phi}^{p}(X ; \mathscr{A}):=H^{p}\left(C_{\Phi}^{*}(X ; \mathscr{A})\right)
$$

Seja $\alpha: \mathscr{A} \rightarrow \mathscr{B}$ um homomorfismo de feixes. Então, existe uma aplicação de resoluções $\mathscr{C}^{*}(X ; \alpha): \mathscr{C}^{*}(X ; \mathscr{A}) \rightarrow \mathscr{C}^{*}(X ; \mathscr{B})$, o que implica a existência de uma aplicação de cocadeias $C_{\Phi}^{*}(X ; \alpha): C_{\Phi}^{*}(X ; \mathscr{A}) \rightarrow C_{\Phi}^{*}(X ; \mathscr{B})$, para qualquer família de suportes $\Phi$. Isso garante a existência de um homomorfismo de módulos

$$
\alpha_{X}^{*}: H_{\Phi}^{*}(X ; \mathscr{A}) \longrightarrow H_{\Phi}^{*}(X ; \mathscr{B}) .
$$

Portanto, quando o espaço $X$ e a família de suportes $\Phi$ são fixados, o funtor $H_{\Phi}^{p}(X ;-): \mathcal{S} \hbar(X) \rightarrow \operatorname{Mod}, \mathscr{A} \mapsto H_{\Phi}^{p}(X ; \mathscr{A})$ associando homomorfismos de feixes $\alpha: \mathscr{A} \rightarrow \mathscr{B}$ a homomorfismos de módulos $\alpha_{X}^{*}$, é chamado cohomologia de feixes. A. Grothendieck em [33, p. 157], usando resoluções injetivas, mostrou que este funtor é o $p$-ésimo funtor derivado à direita do funtor exato à esquerda $\Gamma_{\Phi}$ (vide também [14, Cap. II, Cor. 5.6, p. 49]). Então, como uma consequência do funtor derivado (ou por construção dos grupos de cohomologia) temos a seguinte

Proposição A.2.2. Seja $\Phi$ uma família de suportes sobre $X$. Então,

1. Para todo feixe $\mathscr{A}$ sobre $X$

$$
H_{\Phi}^{0}(X ; \mathscr{A}) \cong \Gamma_{\Phi}(\mathscr{A})
$$


2. Seja $0 \rightarrow \mathscr{A} \rightarrow \mathscr{B} \rightarrow \mathscr{C} \rightarrow 0$ uma sequência exata curta de feixes sobre $X$. Então, existe uma sequência exata longa em cohomologia

$$
\cdots \rightarrow H_{\Phi}^{p}(X ; \mathscr{A}) \rightarrow H_{\Phi}^{p}(X ; \mathscr{B}) \rightarrow H_{\Phi}^{p}(X ; \mathscr{C}) \stackrel{\delta}{\longrightarrow} H_{\Phi}^{p+1}(X ; \mathscr{A}) \rightarrow \cdots
$$

Demonstração. Compare com [14, Cap. II, p. 39 e p 56].

Observação A.2.3. Seja $A$ um subespaço de $X$. Se $\mathscr{A}$ é um feixe sobre $X$ e se $\Psi$ é uma família de suportes sobre $A$, usaremos a notação

$$
H_{\Psi}^{p}(A ; \mathscr{A}):=H_{\Psi}^{p}\left(A ;\left.\mathscr{A}\right|_{A}\right)
$$

A.2.2. Homomorfismos induzidos em cohomologia. Sejam $f: X \rightarrow Y$ uma aplicação contínua, $\mathscr{B}=(\mathscr{B}, \pi)$ um feixe sobre $Y$ e $\Psi$ uma família de suporte sobre $Y$. Considere $\mathscr{C}^{*}(Y ; \mathscr{B})$ e $\mathscr{C}^{*}\left(X ; f^{*} \mathscr{B}\right)$ as resoluções de $\mathscr{B}$ e $f^{*} \mathscr{B}$, respectivamente, ou seja,

$$
\begin{gathered}
\mathscr{C}^{*}(Y ; \mathscr{B}): 0 \longrightarrow \mathscr{B} \stackrel{\epsilon}{\longrightarrow} \mathscr{C}^{0}(Y ; \mathscr{B}) \stackrel{d}{\longrightarrow} \mathscr{C}^{1}(Y ; \mathscr{B}) \stackrel{d}{\longrightarrow} \cdots \\
\mathscr{C}^{*}\left(X ; f^{*} \mathscr{B}\right): 0 \longrightarrow f^{*} \mathscr{B} \stackrel{\epsilon^{*}}{\longrightarrow} \mathscr{C}^{0}\left(X ; f^{*} \mathscr{B}\right) \stackrel{d^{*}}{\longrightarrow} \mathscr{C}^{1}\left(X ; f^{*} \mathscr{B}\right) \stackrel{d^{*}}{\longrightarrow} \cdots
\end{gathered}
$$

Desde que $f^{*}$ é um funtor exato, então $f^{*} \mathscr{C}^{*}(Y ; \mathscr{B})$ dado por:

$$
0 \longrightarrow f^{*} \mathscr{B} \stackrel{f^{*} \epsilon}{\longrightarrow} f^{*} \mathscr{C}^{0}(Y ; \mathscr{B}) \stackrel{f^{*} d}{\longrightarrow} f^{*} \mathscr{C}{ }^{1}(Y ; \mathscr{B}) \stackrel{f^{*} d}{\longrightarrow} f^{*} \mathscr{C}^{2}(Y ; \mathscr{B}) \stackrel{f^{*} d}{\longrightarrow} \cdots
$$

também é uma resolução de $f^{*} \mathscr{B}$, então existe uma aplicação de resoluções ${ }^{1}$ $\xi: \mathscr{C}^{*}\left(X ; f^{*} \mathscr{B}\right) \rightarrow f^{*} \mathscr{C}^{*}(Y ; \mathscr{B})$ sobre o feixe $f^{*} \mathscr{B}$. Portanto, para uma família de suportes $\Phi$ sobre $X$, aplicamos o funtor exato $\Gamma_{\Phi}$ à aplicação $\xi$, obtendo a aplicação de cocadeias

$$
\Gamma_{\Phi}(\xi): \Gamma_{\Phi}\left(f^{*}\left(\mathscr{C}^{*}(Y ; \mathscr{B})\right)\right) \rightarrow \Gamma_{\Phi}\left(\mathscr{C}^{*}\left(X ; f^{*} \mathscr{B}\right)\right)
$$

A passagem à cohomologia garante o seguinte homomorfismo de módulos

$$
H^{p}\left(\Gamma_{\Phi}\left(f^{*} \mathscr{C}^{*}(Y ; \mathscr{B})\right)\right) \longrightarrow H_{\Phi}^{p}\left(X ; f^{*} \mathscr{B}\right)
$$

\footnotetext{
${ }^{1}$ Os métodos de Álgebra Homológica podem ser aplicados à teoria de feixes.
} 
Por outro lado, usando o funtor $\mathscr{A} \rightarrow(U \mapsto \mathscr{A}(U))$ podemos definir o homomorfismo de resoluções de módulos $\mathscr{C}^{*}(Y ; \mathscr{B})(Y) \rightarrow f^{*} \mathscr{C}^{*}(Y ; \mathscr{B})(X)$

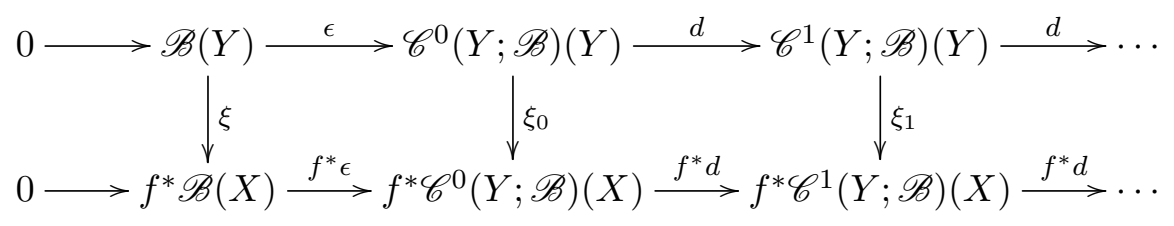

dado por $\xi(s)(x)=f_{x}^{*}(s(f(x)))$ e $\xi_{p}(s)(x)=\bar{f}_{x}^{*}(s(f(x)))$, para todo $p$, onde

$$
f_{x}^{*}: \mathscr{B}_{f(x)} \rightarrow\left(f^{*} \mathscr{B}\right)_{x} \text { e } \bar{f}_{x}^{*}: \mathscr{C}^{p}(Y ; \mathscr{B})_{f(x)} \rightarrow\left(f^{*} \mathscr{C}^{p}(Y ; \mathscr{B})\right)_{x}
$$

são os isomorfismos dados no Exemplo A.1.3(3). Desde que $f_{x}^{*}$ e $\bar{f}_{x}^{*}$ são isomorfismos, é possível mostrar que $|\xi(s)| \subset f^{-1}(|s|)$ e $\left|\xi_{p}(s)\right| \subset f^{-1}(|s|)$, para todo p. Então, se $\Phi$ e $\Psi$ são famílias de suportes tais que $f^{-1}(\Psi) \subset \Phi$, existe uma aplicação de cocadeias

$$
\Gamma(\xi): C_{\Psi}^{*}(Y ; \mathscr{B}) \longrightarrow \Gamma_{\Phi}\left(f^{*} \mathscr{C}^{*}(Y ; \mathscr{B})\right), \quad \Gamma(\xi)=\left\{\xi, \xi_{p}\right\}_{p \in \mathbb{N}}
$$

A passagem à cohomologia garante o seguinte homomorfismo de módulos

$$
H_{\Psi}^{p}(Y ; \mathscr{B}) \longrightarrow H^{p}\left(\Gamma_{\Phi}\left(f^{*} \mathscr{C}^{*}(Y ; \mathscr{B})\right)\right)
$$

Definição A.2.4. Sejam $f: X \rightarrow Y$ uma aplicação contínua e $\mathscr{B}$ um feixe sobre $Y$. Para famílias de suportes $\Phi$ e $\Psi$ sobre $X$ e $Y$, respectivamente, tais que $f^{-1}(\Psi) \subset \Phi$, definimos o homomorfismo ${ }^{1}$ induzido em cohomologia de feixes

$$
f_{\Phi \Psi}^{*}: H_{\Psi}^{p}(Y ; \mathscr{B}) \longrightarrow H_{\Phi}^{p}\left(X ; f^{*} \mathscr{B}\right)
$$

como sendo a composta de (A.9) e (A.11).

A seguir apresentaremos as propriedades básicas do homomorfismo induzido. Proposição A.2.5. Sejam $f: X \rightarrow Y$ e $g: Y \rightarrow Z$ aplicações contínuas. Se $\Phi, \Psi$ e $\Omega$ são famílias de suportes sobre $X, Y$ e $Z$, respectivamente, tais que $f^{-1}(\Psi) \subset \Phi$ e $g^{-1}(\Omega) \subset \Psi$, então $(g \circ f)_{\Phi \Omega}^{*}$ é a composta

$$
H_{\Omega}^{p}(Z ; \mathscr{C}) \stackrel{g_{\Psi \Omega}^{*}}{\longrightarrow} H_{\Psi}^{p}\left(Y ; g^{*} \mathscr{C}\right) \stackrel{f_{\Phi \Psi}^{*}}{\longrightarrow} H_{\Phi}^{p}\left(X ; f^{*}\left(g^{*} \mathscr{C}\right)\right) .
$$

\footnotetext{
${ }^{1}$ Homomorfismo de módulos.
} 
Demonstração. Basta provar que $f^{*}\left(g^{*} \mathscr{C}\right) \simeq(g \circ f)^{*} \mathscr{C}$.

Proposição A.2.6. Sejam $\operatorname{Id}_{X}: X \rightarrow X$ a identidade e $\Phi$ uma família de suportes sobre X. Então,

$$
\operatorname{Id}=\left(\operatorname{Id}_{X}\right)_{\Phi \Phi}^{*}: H_{\Phi}^{p}(X ; \mathscr{A}) \longrightarrow H_{\Phi}^{p}(X ; \mathscr{A})
$$

Demonstração. Basta provar que $\operatorname{Id}_{X}^{*} \mathscr{A} \simeq \mathscr{A}$.

Corolário A.2.7. Seja $f: X \rightarrow Y$ um homeomorfismo, com $f^{-1}(\Psi)=\Phi$. Então, $f_{\Phi \Psi}^{*}: H_{\Psi}^{p}(Y ; \mathscr{B}) \longrightarrow H_{\Phi}^{p}\left(X ; f^{*} \mathscr{B}\right)$ é um isomorfismo, para todo feixe $\mathscr{B}$.

Demonstração. Basta provar que $g_{\Psi \Phi}^{*}: H_{\Phi}^{p}\left(X ; f^{*} \mathscr{B}\right) \longrightarrow H_{\Psi}^{p}(Y ; \mathscr{B})$ é a inversa de $f_{\Phi \Psi}^{*}$, onde $g$ é a inversa de $f$.

A.2.3. Módulos de cohomologia relativa. Sejam $A$ um subespaço de $X$ e $\mathscr{A}$ um feixe sobre $X$. A correspondência $\left.U \mapsto \mathscr{A}\right|_{A}(U \cap A)=\left.\mathscr{A}\right|_{A}\left(\imath^{-1}(U)\right)$ define um prefeixe sobre $X$, onde $\imath: A \hookrightarrow X$ é a inclusão. Este prefeixe satisfaz a condição A.2 e, portanto, é um feixe ${ }^{1}$ que será denotado por $\left.\imath \mathscr{A}\right|_{A}$. Pela Observação A.1.12, $\left.\left.\imath \mathscr{A}\right|_{A}(U) \cong \mathscr{A}\right|_{A}(U)$, para todo aberto $U \subset X$.

Seja $U$ um subconjunto aberto de $X$, então temos a sequência exta curta

$$
0 \longrightarrow \prod_{x \in U-A} \mathscr{A}_{x} \times \prod_{x \in U \cap A} 0 \longrightarrow \prod_{x \in U} \mathscr{A}_{x} \stackrel{p}{\longrightarrow} \prod_{x \in U \cap A} \mathscr{A}_{x} \longrightarrow 0
$$

onde $p$ é a projeção sobre $\prod_{x \in U \cap A} \mathscr{A}_{x}$. Portanto, temos um diagrama comutativo

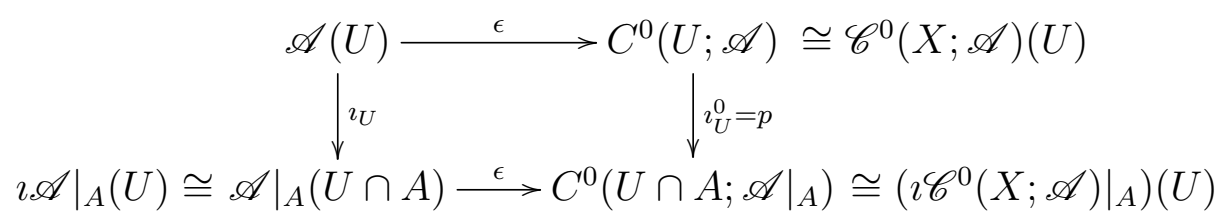

$\operatorname{com} \imath_{U}^{0}$ sobrejetor. Continuando indutivamente, temos a aplicação de resoluções $\imath^{*}: \mathscr{C}^{*}(X ; \mathscr{A}) \rightarrow \imath \mathscr{C}^{*}(A ; \mathscr{A} \mid A)$ dada por

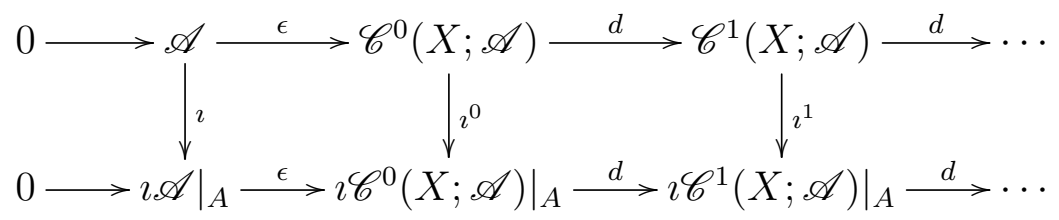

\footnotetext{
${ }^{1}$ Uma generalização deste feixe pode ser encontrada em [14, Cap. I, Seção §3, p. 12].
} 
$\operatorname{com} \imath^{p}$ sobrejetor, para todo $p$. Logo,

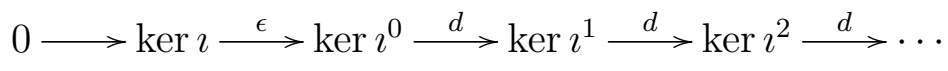

define outra resolução do subfeixe ker $\imath$ de $\mathscr{A}$, denotada por $\mathscr{C}^{*}(X, A ; \mathscr{A})$.

Agora, seja $\Phi$ uma família de suportes sobre $X$. Aplicando o funtor $\Gamma_{\Phi}$ a $\mathscr{C}^{*}(X, A ; \mathscr{A})$ obtemos o complexo de cocadeias

$$
C_{\Phi}^{*}(X, A ; \mathscr{A})=\Gamma_{\Phi}\left(\mathscr{C}^{*}(X, A ; \mathscr{A})\right)
$$

Definição A.2.8. Seja $A$ um subespaço de $X$. Para uma família $\Phi$ de suportes sobre $X$ e para um feixe $\mathscr{A}$ sobre $X$, definimos o p-ésimo módulo de cohomologia relativo do par $(X ; A)$, com coeficientes em $\mathscr{A}$ e suporte em $\Phi$ por

$$
H_{\Phi}^{p}(X, A ; \mathscr{A}):=H^{p}\left(C_{\Phi}^{*}(X, A ; \mathscr{A})\right) .
$$

Pela construção, temos que $H_{\Phi}^{p}(X, \emptyset ; \mathscr{A})=H_{\Phi}^{p}(X ; \mathscr{A})$. Além disso, temos o seguinte resultado cuja prova pode ser encontrada em [14, Cap. II, p. 84].

Proposição A.2.9. Seja $\Phi$ uma família de suporte sobre $X$. Então, existe uma sequência exata longa em cohomologia do par $(X, A)$

$$
\cdots \longrightarrow H_{\Phi}^{p}(X ; \mathscr{A}) \longrightarrow H_{\Phi \cap A}^{p}\left(A ;\left.\mathscr{A}\right|_{A}\right) \stackrel{\Delta}{\longrightarrow} H_{\Phi}^{p+1}(X, A ; \mathscr{A}) \longrightarrow \cdots
$$

Analogamente, como no caso absoluto, podemos definir o homomorfismo induzido em cohomologia relativa da aplicação de pares $f:(X, A) \rightarrow(Y, B)$ (i.e. uma aplicação continua $f: X \rightarrow Y$ tal que $f(A) \subset B)$,

$$
f_{\Phi \Psi}^{*}: H_{\Psi}^{p}(Y, B ; \mathscr{B}) \longrightarrow H_{\Phi}^{p}\left(X, A ; f^{*} \mathscr{B}\right)
$$

com as mesmas características descritas na Proposição A.2.5 e no Corolário A.2.7.

ObservaÇão A.2.10. Para pares paracompactos fechados, os módulos de cohomologia com coeficientes constantes e suporte fechado satisfazem os axiomas de Eilenberg-Steenrod e, neste caso, coincidem com a teoria de cohomologia singular sobre CW-complexos. 


\section{A.3. Homologia de Borel-Moore}

Daqui em diante assumiremos que $X$ é um espaço de Hausdorff localmente compacto e $L$ um domínio de ideais principais. Nessas condições, a família de suporte compacto $\boldsymbol{c}$ sobre $X$ é paracompactificante (vide Exemplo A.1.15-1).

A.3.1. Módulos de homologia. Seja $\hat{L}=\operatorname{Hom}_{\mathbb{Z}}(L ; T)$, onde $T=\mathbb{Q} / \mathbb{Z}$. Desde que $T$ é injetivo, o homomorfismo de módulos $L \rightarrow \operatorname{Hom}_{\mathbb{Z}}(\hat{L} ; T), a \mapsto a^{*}$, onde $a^{*}(f)=f(a)$, é um monomorfismo. Agora, seja $F(\hat{L})$ o módulo livre sobre os elementos de $L$. Então existe um homomorfismo de módulos sobrejetor $F(\hat{L}) \rightarrow \hat{L}$ e, portanto, um monomorfismo de módulos natural

$$
\operatorname{Hom}_{\mathbb{Z}}(\hat{L} ; T) \rightarrow \operatorname{Hom}_{\mathbb{Z}}(F(\hat{L}) ; T)
$$

A composição $L \longmapsto \operatorname{Hom}_{\mathbb{Z}}(\hat{L} ; T) \longmapsto \operatorname{Hom}_{\mathbb{Z}}(F(\hat{L}) ; T)$ é então um monomorfismo no módulo injetivo $I(L)=\operatorname{Hom}_{\mathbb{Z}}(F(\hat{L}) ; T)$.

Para cada subconjunto aberto $U$ de $X$, seja o módulo

$$
\bar{C}^{0}(U ; L)=\prod_{x \in U} I\left(L_{x}\right), \text { onde } L_{x}=L
$$

para todo $x \in U$. O prefeixe $U \mapsto \bar{C}^{0}(U ; L)$ define um feixe ${ }^{1}$ injetivo sobre $X$, denotado por $\mathscr{I}^{0}(X ; L)$. Desde que $I\left(L_{x}\right)$ contém $L$, então temos a inclusão

$$
C^{0}(U ; L)=\prod_{x \in U} L_{x} \hookrightarrow \prod_{x \in U} I\left(L_{x}\right)=\bar{C}^{0}(X ; L)
$$

que fornece um monomorfismo de feixes $\mathscr{C}^{0}(X ; L) \longmapsto \mathscr{I}^{0}(X ; L)$. Compondo com o monomorfismo $\epsilon: L \longmapsto \mathscr{C}^{0}(X ; L)$, temos um monomorfismo $L \rightarrow \mathscr{I}^{0}(X ; L)$. Continuando da mesma forma como foram construídos os feixes $\mathscr{C}^{p}(X ; \mathscr{A})$, obtemos uma resolução.

$$
\mathscr{I}^{*}: 0 \longrightarrow L \longrightarrow \mathscr{I}^{0}(X ; L) \longrightarrow \mathscr{I}^{1}(X ; L) \longrightarrow \mathscr{I}^{2}(X ; L) \longrightarrow \cdots
$$

onde cada feixe $\mathscr{I}^{p}=\mathscr{I}^{p}(X ; L)$ é injetivo contendo $\mathscr{C}^{p}(X ; L)$, para todo $p$.

\footnotetext{
${ }^{1}$ Da mesma forma como na construção dos módulos $\mathscr{C}^{0}(X ; \mathscr{A})$.
} 
Lema A.3.1. Para cada $p$ e para cada cobertura $\left\{U_{\alpha}\right\}$ de um subconjunto aberto $U$ de $X$, a sequência

$$
\bigoplus_{\langle\alpha, \beta\rangle} \Gamma_{c}\left(\left.\mathscr{I}^{p}\right|_{U_{\alpha} \cap U_{\beta}}\right) \stackrel{g}{\longrightarrow} \bigoplus_{\alpha} \Gamma_{c}\left(\left.\mathscr{I}^{p}\right|_{U_{\alpha}}\right) \stackrel{h}{\longrightarrow} \Gamma_{c}\left(\left.\mathscr{I}^{p}\right|_{U}\right) \longrightarrow 0
$$

é exata, onde

1. $g=\sum_{\langle\alpha, \beta\rangle}\left(\Gamma_{c}\left(\imath_{U_{\alpha}, U_{\alpha} \cap U_{\beta}}\right)-\Gamma_{c}\left(\imath_{U_{\beta}, U_{\alpha} \cap U_{\beta}}\right)\right)$, sendo $\imath_{U_{\alpha}, U_{\alpha} \cap U_{\beta}}:\left.\left.\mathscr{I}^{p}\right|_{U_{\alpha} \cap U_{\beta}} \hookrightarrow \mathscr{I}^{p}\right|_{U_{\alpha}}$.

2. $h:=\sum_{\alpha} \Gamma_{c}\left(\imath_{U, U_{\alpha}}\right)$, sendo $\imath_{U, U_{\alpha}}:\left.\left.\mathscr{I}^{p}\right|_{U_{\alpha}} \hookrightarrow \mathscr{I}^{p}\right|_{U}$.

Demonstração. Segue de [14, Cap. II, Prop. 5.3 (p. 48) e Cor. 9.6 (p. 66)] e [14, Cap. V, Prop. 1.6, p. 282].

Aplicando o funtor $\Gamma_{c}$ à resolução $\left.\mathscr{I}^{*}\right|_{U}$, para um aberto $U \subset X$, obtemos o complexo de cocadeias $\Gamma_{c}\left(\left.\mathscr{I}^{*}\right|_{U}\right)$. Usando a graduação $\left(\Gamma_{c}\left(\left.\mathscr{I}^{*}\right|_{U}\right)\right)_{p}:=\Gamma_{c}\left(\left.\mathscr{I}^{-p}\right|_{U}\right)$, temos que $\Gamma_{c}\left(\left.\mathscr{I}^{*}\right|_{U}\right)$ pode ser visto como um complexo de cadeias, ou seja

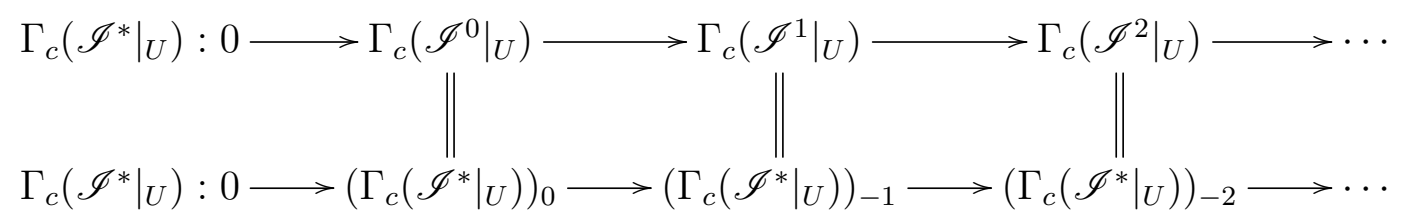

Desde que $L$ é um PID, existe uma resolução injetiva $R(L)$ de $L$

$$
0 \rightarrow L \rightarrow L^{0} \rightarrow L^{1} \rightarrow \cdots L^{q} \rightarrow \cdots
$$

onde $L^{0}=L^{*}$ (corpo de frações), $L^{1}=L^{*} / L$ e $L^{q}=0$, para $q \neq 0,1$. Por outro lado, segue do Lema A.3.1 e de [14, Cap. V, Prop. 1.10, p. 285] que o prefeixe

$$
U \mapsto \operatorname{Hom}\left(\Gamma_{c}\left(\left.\mathscr{I}^{p}\right|_{U}\right) ; M\right)
$$

é um feixe sobre $X$, denotado por $\mathcal{H o m}\left(\Gamma_{c} \mathscr{I}^{p} ; M\right)$, para todo $p$, onde $M$ é um módulo fixado tal que as restrições $r_{U, V}$ são induzidas pela inclusão

$$
\Gamma_{c}\left(\left.\mathscr{I}^{p}\right|_{U}\right) \rightarrow \Gamma_{c}\left(\left.\mathscr{I}^{p}\right|_{V}\right)
$$

para $U \subset V$. Considerando $\Gamma_{c}\left(\left.\mathscr{I}^{*}\right|_{U}\right)$ como complexo de cadeias, definimos o 
feixe graduado $\mathscr{D}\left(\Gamma_{c} \mathscr{I}^{*} ; L\right)$

$$
\begin{aligned}
& \mathscr{D}_{0}:=\operatorname{Sheaf}\left(U \mapsto \operatorname{Hom}\left(\left(\Gamma_{c}\left(\left.\mathscr{I}^{*}\right|_{U}\right)\right)_{0} ; L^{0}\right) \oplus \operatorname{Hom}\left(\left(\Gamma_{c}\left(\left.\mathscr{I}^{*}\right|_{U}\right)\right)_{-1} ; L^{1}\right)\right) \\
& =\operatorname{Hom}\left(\Gamma_{c} \mathscr{I}^{0} ; L^{0}\right) \oplus \mathscr{H o m}\left(\Gamma_{c} \mathscr{I}^{1} ; L^{1}\right) \\
& \mathscr{D}_{-1}:=\operatorname{Sheaf}\left(U \mapsto \operatorname{Hom}\left(\left(\Gamma_{c}\left(\left.\mathscr{I}^{*}\right|_{U}\right)\right)_{-1} ; L^{0}\right) \oplus \operatorname{Hom}\left(\left(\Gamma_{c}\left(\left.\mathscr{I}^{*}\right|_{U}\right)\right)_{-2} ; L^{1}\right)\right) \\
& =\operatorname{Hom}\left(\Gamma_{c} \mathscr{I}^{1} ; L^{0}\right) \oplus \mathscr{H o m}\left(\Gamma_{c} \mathscr{I}^{2} ; L^{1}\right) \\
& \vdots \\
& \mathscr{D}_{-n}:=\operatorname{Sheaf}\left(U \mapsto \operatorname{Hom}\left(\left(\Gamma_{c}\left(\left.\mathscr{I}^{*}\right|_{U}\right)\right)_{-n} ; L^{0}\right) \oplus \operatorname{Hom}\left(\left(\Gamma_{c}\left(\left.\mathscr{I}^{*}\right|_{U}\right)\right)_{-n-1} ; L^{1}\right)\right) \\
& =\operatorname{Sheaf}\left(U \mapsto \bigoplus_{p+q=-n} \operatorname{Hom}\left(\left(\Gamma_{c}\left(\left.\mathscr{I}^{*}\right|_{U}\right)\right)_{p} ; L^{q}\right)\right. \\
& =\bigoplus_{p+q=-n} \mathcal{H} \operatorname{fom}\left(\Gamma_{c} \mathscr{I}^{-p} ; L^{q}\right)
\end{aligned}
$$

Portanto, podemos definir os homomorfismos de feixes $d: \mathscr{D}_{-n} \rightarrow \mathscr{D}_{-n-1}$ por $d=d^{\prime}-d^{\prime \prime}$, sendo $d^{\prime}$ o homomorfismo induzido pelo diferencial $L^{q} \rightarrow L^{q+1} \mathrm{e}$ $(-1)^{n} d$ " sendo o homomorfismo induzido por $\Gamma_{c}\left(\left.\mathscr{I}^{-p-1}\right|_{U}\right) \rightarrow \Gamma_{c}\left(\left.\mathscr{I}^{-p}\right|_{U}\right)$, para todo aberto $U \subset X$. Considerando a notação $\mathscr{D}^{n}=\mathscr{D}_{-n}$ e desde que $d \circ d=0$ temos o feixe diferencial

$$
\mathscr{D}\left(\Gamma_{c} \mathscr{I}^{*} ; L\right): 0 \longrightarrow \mathscr{D}^{0} \stackrel{d}{\longrightarrow} \mathscr{D}^{1} \stackrel{d}{\longrightarrow} \mathscr{D}^{2} \stackrel{d}{\longrightarrow} \cdots
$$

Seja $\mathscr{A}$ um feixe sobre $X$. Denotaremos por $\mathscr{C}_{*}(X ; \mathscr{A})$ o feixe diferencial

$$
\mathscr{C}_{*}(X ; \mathscr{A})=\mathscr{D}\left(\Gamma_{c} \mathscr{I}^{*} ; L\right) \otimes \mathscr{A}
$$

Agora, seja $\Phi$ uma família de suportes sobre $X$. Aplicando o funtor $\Gamma_{\Phi}$ a $\mathscr{C}_{*}(X ; \mathscr{A})$ obtemos o complexo de cocadeias

$$
C_{*}^{\Phi}(X ; \mathscr{A}):=\Gamma_{\Phi}\left(\mathscr{C}_{*}(X ; \mathscr{A})\right)
$$

Definição A.3.2. Seja $X$ um espaço Hausdorff localmente compacto. Para uma família $\Phi$ de suportes sobre $X$ e para um feixe $\mathscr{A}$ sobre $X$, definimos o $p$-ésimo módulo de homologia de $X$ com coeficientes em $\mathscr{A}$ e suporte em $\Phi$ por

$$
H_{p}^{\Phi}(X ; \mathscr{A}):=H_{p}\left(C_{*}^{\Phi}(X ; \mathscr{A})\right)
$$


Não é imediato provar que $H_{-1}^{\Phi}(X ; \mathscr{A})=0$. Para uma família paracompactificante este fato foi provado em [14, Cap. V, Teo. 5.13,p. 313].

Proposição A.3.3. Seja $\Phi$ uma família de suportes sobre $X$ paracompactificante. Seja $0 \rightarrow \mathscr{A} \rightarrow \mathscr{B} \rightarrow \mathscr{C} \rightarrow 0$ uma sequência exata curta de feixes sobre $X$, então existe uma sequência exata longa em cohomologia

$$
\cdots \rightarrow H_{p}^{\Phi}(X ; \mathscr{A}) \rightarrow H_{p}^{\Phi}(X ; \mathscr{B}) \rightarrow H_{p}^{\Phi}(X ; \mathscr{C}) \stackrel{\delta}{\longrightarrow} H_{p-1}^{\Phi}(X ; \mathscr{A}) \rightarrow \cdots
$$

Demonstração. Vide [14, Eq. (8), p. 292].

A.3.2. Homomorfismo induzido em homologia. Seja $f: X \rightarrow Y$ uma função contínua entre espaços localmente compactos Hausdorff. Desde que $f^{*}$ preserva feixes constantes, ou seja, $f^{*} Y \times L \simeq X \times L$, segue de (A.8) e (A.10) que existe uma aplicação de cocadeias

$$
f^{*}: C_{c}^{*}(Y ; L) \rightarrow C_{c}^{*}(X ; L)
$$

a qual é unica, a menos de homotopia, e induz a aplicação

$$
D f^{*}: \mathscr{D}\left(C_{c}^{*}(X ; L)\right) \rightarrow \mathscr{D}\left(C_{c}^{*}(Y ; L)\right)
$$

Desde que $\mathscr{D}\left(C_{c}^{*}(X ; L)\right)=\Gamma\left(\mathscr{C}_{*}(X ; L)\right)$ e $\mathscr{D}\left(C_{c}^{*}(Y ; L)\right)=\Gamma\left(\mathscr{C}_{*}(Y ; L)\right)$, então existe um homomorfismo de módulos

$$
f_{*}: H_{p}(X ; L) \longrightarrow H_{p}(Y ; L) \text {. }
$$

Para coeficientes em um feixe qualquer, essa construção pode ser encontrada em [14, Cap. V, Seção §4].

A.3.3. Módulos de homologia relativa. Sejam $A \subset X$ um subespaço localmente fechado $^{1}$ (e, portanto, localmente compacto) e $\Phi$ uma família de suportes sobre $X$. Pode-se provar que a inclusão $\imath: A \rightarrow X$ induz um monomorfismo

$$
C_{*}^{\left.\Phi\right|_{A}}\left(A ;\left.\mathscr{A}\right|_{A}\right) \longrightarrow C_{*}^{\Phi}(X ; \mathscr{A})
$$

\footnotetext{
${ }^{1}$ Um subconjunto $A \subset X$ é localmente fechado se, para todo $a \in A$, existe uma vizinhança aberta $U \subset X$ de $x$ tal que $U \cap A$ é fechado em $U$.
} 
(vide [14, Cap. V, p. 303-305]). Defina

$$
C_{*}^{\Phi}(X, A ; \mathscr{A}):=\frac{C_{*}^{\Phi}(X ; \mathscr{A})}{C_{*}^{\left.\Phi\right|_{A}}\left(A ;\left.\mathscr{A}\right|_{A}\right)} .
$$

Definição A.3.4. Seja $A \subset X$ um subespaço localmente fechado. Para uma família $\Phi$ de suportes sobre $X$ e para um feixe $\mathscr{A}$ sobre $X$, definimos o p-ésimo módulo de homologia relativo do par $(X ; A)$, com coeficientes em $\mathscr{A}$ e suporte em $\Phi$ por

$$
H_{p}^{\Phi}(X, A ; \mathscr{A}):=H_{p}\left(C_{*}^{\Phi}(X, A ; \mathscr{A})\right)
$$

Observação A.3.5. Para pares localmente compactos, os módulos de homologia com coeficientes constantes e suporte compacto satisfazem os axiomas de Eilenberg-Steenrod e, neste caso, coincidem com a teoria de homologia singular sobre CW-complexos localmente finitos. 


\section{B}

\section{Notas históricas e bibliográficas}

O propósito deste Apêndice é apresentar um pequeno resumo do surgimento das variedades generalizadas. Na verdade, os resultados apresentados aqui são uma coletânea dos muitos fatos encontrados em alguns dos artigos descritos nas Referências Bibiliográficas.

Variedades generalizadas A partir do ano de 1925, os topólogos começaram a desenvolver uma extensão da noção de grupos de homologia para espaços não necessariamente triánguláveis. Duas idéais surgiram: a primeira, a homologia singular, foi introduzida por topólogos da Universidade de Princeton e definida com a máxima generalidade por Samuel Eilenberg. Outros métodos foram dados por Pavel Sergeevich Aleksandrov, Leopold Vietoris e Solomon Lefschetz que definiram, independentemente, módulos de homologia para espaços métricos compactos com coeficientes em $\mathbb{Z}, \mathbb{Q}$ e $\mathbb{F}_{p}$ (corpo finito com $p$ elementos, sendo $p$ primo). Eduard $\check{C}$ ech generalizou o método de P. Aleksandrov para espaços topológicos gerais. ${ }^{1}$. Quase ao mesmo tempo, a teoria de dimensão foi introduzida por Pavel Sergeevich Urysohn, Karl Menger, Luitzen Egbertus Jan Brouwer e Lebesgue ${ }^{2}$.

Essas teorias foram posteriormente estendidas para uma classe mais ampla de espaços por S. Lefschetz [45] e E. Čech [16] (por volta de 1933). Resultados importantes, que antes haviam sido provado apenas para variedades topológicas compactas trianguláveis orientadas, foram então obtidos para variedades topológicas, entre eles, os Teoremas de Dualidade (de Poincaré e Alexander), Ambos

\footnotetext{
${ }^{1}$ Alguns autores a chamam homologia de Aleksandrov-Čech.

${ }^{2}$ Atualmente, são os conecitos de dimensão indutiva e dimensão topológica.
} 
utilizaram métodos distintos em seus trabalhos de pesquisa ${ }^{1}$ usando suas respectivas teorias de homologia, descritas anteriormente. Na verdade, E. Čech lidou com um tipo mais geral de espaços, definindo axiomaticamente uma variedade generalizada. No entanto, Lefschetz restringiu sua definição [45, Def. 22, p. 487] a espaços métricos localmente compactos, o que lhe permitiu estender os resultados de $[28,29,44]$ a variedades generalizadas. Ambos utilizaram a dimensão de Menger-Urysohn em suas definições. Além disso, em [16, Cap. VII] e [45, p. 504] surgiram as primeiras demonstrações em relação aos Teoremas de Dualidade desses espaços.

Quase simultaneamente, tendo conseguido estender a recíproca do Teorema da Separação de Jordan-Brouwer ao espaço tridimensional (vide [60]), Raymond Louis Wilder publicou [61], em 1934, uma caracterização, por propriedades intrínsecas de homologia, do conjunto de pontos do espaço $\mathbb{R}^{n}$ que é fronteira de um único domínio satisfazendo certas condições. O resultado é a definição de variedade fechada generalizada [61, §1, p. 878], dando continuidade ao estudo das variedades generalizadas iniciado por S. Lefschetz e E. Čech. Em sua definição, Wilder utilizou a dimensão de Brouwer-Urysohn-Menger e os ciclos de Vietoris. Nesse mesmo artigo, foi provado apenas o Teorema da Dualidade de Poincaré para variedades fechadas generalizadas $M \subset \mathbb{R}^{m}[61$, §2, Teo. 6, p. 886].

Edward Griffith Begle, em sua tese de doutorado de 1940 (cujos resultados apareceram posteriormente publicados em [1] de 1942), trabalhou com os conceitos de realização e realização parcial de um complexo finito sobre um espaço que tinha sido definido pelo seu orientador S. Lefschetz em [46]. Usando também os ciclos de Vietoris, ele deu novas definições desses conceitos, o que lhe permitiu estender os métodos desenvolvidos antes para espaços LC (espaços localmente conexos, no sentido de homotopia), agora para espaços lc (espaços localmente conexos, no sentido de homologia). Além disso, ele também demonstrou que os números de Betti locais (definidos por ciclos de Vietoris) podiam ser formulados em termos de cociclos, chamando-os números de co-Betti locais. Tendo estudado estes dois invariantes, que foram usados nas definições anteriores de variedade generalizada, E. G. Begle obteve uma nova definição [1, Def. 7.1, p. 568] desses espaços ${ }^{2}$ e simplificou seu estudo. Em [1, Teo. 7.3, p. 570], apresentou uma pequena prova

\footnotetext{
${ }^{1}$ Vide nota de rodapé 6 e $\ddagger$ em [16, p. 622] e [45, p. 469], respectivamente.

${ }^{2}$ Equivalente à definição de E. Čech e coincidindo com a definição de R. Wilder quando o espaço for métrico (vide [1, Teo. 8.1] e nota de rodapé $n^{o} 3$ em [2, p. 59], respectivamente.)
} 
do teorema de Dualidade de Poincaré, usando cociclos. Em seu artigo de 1945, essa definição foi modificada para espaços localmente compactos, substituindo a condição do espaço ser $\mathrm{lc}^{n-1}$, pela condição que os números de co-Betti locais $R_{p}(s)$ fossem nulos, para $p<n$ e para todo ponto, sendo chamada de variedade generalizada aberta [2, Def. 7.1, p. 568]. Isso permitiu demonstrar os Teoremas de Dualidade de Poincaré e Alexander [2, Teo. 5.1, p. 64 e Teo. 6.4, p. 68].

Em seu famoso livro de 1949 [62], R. Wilder aperfeiçoou esta última definição, eliminando a condição desnecessária do espaço ser $1 c^{n-1}$ e trocando a condição que os números de co-Betti locais $R_{p}(s)$ sejam nulos, para $p<n$, pela condição equivalente do espaço ser $\operatorname{col}^{n-1}$ (co-conexidade local), obtendo a clássica definição de variedade generalizada (vide [62, Cap. VI, Teo. 6.10, p. 192 e Cap. VIII, p. 244]). Em sua definição, ele usou o conceito de dimensão topológica. A definição de variedade generalizada segundo Wilder é a seguinte:

General properties. A locally compact space $S$ will be called a generalized manifold of dimension $n$ if the following axioms are satisfied: A.) The dimension of $S$ is $n, B$.) $S$ is colc ${ }^{n-1}$, C.) For each $x \in S, p_{n}(x)=1$.

Em 1942, Lefschetz [43, p. 273] definiu para espaços métricos compactos um isomorfismo entre os grupos de homologia de Čech e os grupos de homologia de Vietoris, fato que permitiu relacionar a definição de Wilder com as anteriores para espaços métricos compactos. As provas dos Teoremas de Dualidade nesta referência são as provas de E. G. Begle modificadas pelo autor [62, Cap. VIII, Seções $4,6,7,9]$.

Até então, nenhuma informação havia sido dada em relação a variedades generalizadas com bordo. Esse conceito foi introduzido por Paul Alexander White [59, Def. 5, p. 239] (1950) para espaços compactos. Para espaços localmente compactos, foi introduzido por Thomas Roy Brahana [8, p. 35] (1957).

Em 1946, Jean Leray introduziu a teoria de feixes (vide [41, 42]), que posteriormente foi aperfeiçoada por Michel Lazard e Alexander Grothendieck. O primeiro autor definiu um feixe como sendo um espaço topológico e o segundo introduziu o conceito de prefeixe, obtendo assim uma versão funtorial de feixe, equivalente à definição dada por M. Lazard (vide [15, Exp. no. 14] e [32, 33]). Com este novo conceito, o aluno de J. Leray, Armand Borel [3] (1957), levou a prova de R. L. Wilder do Teorema da Dualidade de Poincaré ao âmbito da teoria de feixes, construindo uma teoria de homologia equivalente à teoria de homologia de Čech (sobre corpos). Em seu artigo, ele utilizou a cohomologia de 
Alexander-Spanier, e para definir os números de co-Betti locais, que simplesmente chamou de números de Betti locais, utilizou a cohomologia de Alexander-Spanier com suporte compacto. A dimensão considerada por A. Borel foi a $\Phi$-dimensão cohomológica introduzida em [15], e na definição de variedade generalizada [3, Def. 7., p. 235] somente considerou o caso finito. Este novo conceito de dimensão estava relacionada com as definições anteriores.

Até aqui, na teoria das variedades generalizadas, o anel de coeficientes tinha sido um corpo. Devido à influência dos grupos de transformações, A. Borel em [4, Cap. I, Seção §3] (1960) estendeu sua definição anterior para coeficientes em um domínio de ideias principais (PID). Os espaços obtidos foram chamados variedades de Wilder. No caso em que as variedades de Wilder são localmente orientáveis (conceito também definido para um PID), elas foram chamadas variedades de cohomologia. Em [4, Cap. II, Seção §1, p. 24] Borel construiu o feixe de homologia local com fibras $H_{i}^{x}(X ; L)$ sobre um PID $L$. Usando a Fórmula dos Coeficientes Universais, ele estendeu o Teorema da Dualidade de Poincaré em [3] para o seguinte espaço (vide [4, Cap. II, Seção §2]) :

2.1. Definition. The space $X$ is $L-n$ space if $\operatorname{dim}_{L}(X)$ is finite and if for any $x \in X$, we have $H_{i}^{x}(X ; L)=0(i \neq n)$ and $H_{n}^{x}(X ; L)=L$. An $L$-n space is locally orientable (respectively orientable) if the local homology sheaf is locally simple (respectively simple).

Se $L$ é um corpo os $L-n$ espaços localmente orientáveis coincidem com as variedades de cohomologia e, portanto, coincidem com a noção original de Wilder. As variedades generalizadas com bordo sobre um PID foram introduzidas por Frank Raymond [53, Def. 3, p. 9].

Em 1960, Borel e Moore, em [5], aprimoraram a teoria de homologia que Borel havia construído, generalizando a definição dos $L$ - $n$ espaços, que foram chamados variedades de homologia, para as quais o Teorema de Dualidade de Poincaré é satisfeito. Esta foi a definição considerada em nosso trabalho. 


\section{Referências Bibliográficas}

[1] Begle, E.; Locally connected spaces and generalized manifolds. American Journal of Mathematics Vol. 64 (1942), no. 1, 553-574.

[2] _ ; Duality theorems for generalized manifolds, American Journal of Mathematics 67 (1945), no. 1, 59-70.

[3] Borel, A.; The poincaré duality in generalized manifolds. Michigan Mathematics Journal 4 (1957), 227-239.

[4] __ et al.; Seminar on transformation groups. Annals of mathematics studies, Princeton University Press, 1960.

[5]_ and Moore, J.; Homology theory for locally compact spaces. Michigan Mathematical Journal, 7 (1960), 137-159.

[6] Borsuk, Karol; Uber Zerlengung einer euklidischen n-dimensionalen Volkugeln in $n$ Mengen, Verhandlungen des Internationalen Mathematiker Kongresses, Zürich, (1932); II. Band: Sekt.-Vortäge, Orel Fusli, Zürich, (1932), 142-198.

[7] ___ Drei Sätze über die n-dimensionale euklidische Sphäre, Fundamentia Maathematicae 20 (1933), 177-190 
[8] Brahana, T.; A theorem about local Betti groups. Michigan Mathematical Journal, Vol. 4 (1957), 33-37.

[9] Bredon, G.; Cohomology fibre spaces, the smith-gysin sequence, and orientation in generalized manifolds. Michigan Mathematics Journal, 10 (1963), $321-333$.

[10] _ _ Cosheaves and homology. Pacific Journal of Mathematics , Vol. 25 (1968), 1-32.

[11]__ ; Wilder manifolds are locally orientables. Proceedings of the National Academy of Sciences of the United States of America, Vol. 63 (1969), 10791081

[12] _ ; Generalized manifolds, revisited. Topology of Manifolds, Markham Publ. Co., Chicago (1970), 461-469.

[13] _ Introduction to compact transformation groups, Pure and Applied Mathematics, Elsevier Science, 1972.

[14] _ ; Sheaf theory, 2nd ed., Graduate Texts in Mathematics, Vol. 170, Springer, New York, 1997.

[15] Cartan, H.; Cohomologie des groupes, suite spectral, faisceaux. Seminaire de Topologie algébrique de l'École Normale Supérieure, 1948-49, 1950-51.

[16] Čech, E.; Théorie générale des variétés et de leurs théorèmes de dualité. Annals of Mathematics, Second Series, Vol. 34 (1933), no. 4, 621-730.

[17] Cohen, H.; A cohomological definition of dimension for locally compact Hausdorf spaces. Duke Mathematics Journal, Vol. 21 (1954), 209-224.

[18] Conner, P. and Floyd, E., A characterization of generalized manifolds, Michigan Mathematical Journal 6 (1959), 33-43.

[19] Cordova, N. , de Mattos, D. , dos Santos, E.L. , Degree of equivariant maps between generalized $G$-manifolds, Journal of Fixed Point Theory and Applications 13 (2013), 163-173.

[20] Daccach, J.; Nonexistence of equivariant degree one maps. Proceedings of the American Mathematical Society, Vol. 101 (1987), 530-532. 
[21] Deo, S.; Sheaf theoretic cohomological dimension and finitistic spaces. Proceeding of the American Mathematical Society, Vol. 86 (1982), no. 3, 545-550.

[22] Dold, A.; Simple proofs of some Borsuk-Ulam results, Contemp. Math., vol. 19, Amer. Math. Soc., Providence, R.I., 1983, 65-69.

[23] Dieck, T.; Transformation groups and representation theory, Lectures Notes in Mathematics 766, Springer-Verlag, Berlin 1979.

[24] Eilenberg, S.; On the theorem of P. A. Smith concerning fixed points for periodic maps., Duke Mathematical Journal 6 (1940), 428-437.

[25] Engelking, R.; General Topology. Warszawa, 1977.

[26] Epstein, D.; The degree of a map. Proceeding of the London Mathematical Society, Vol. 16 (1966), no. 3, 7-21.

[27] Fadell, E.R. and Huseini, S., An ideal-valued cohomological index theory with applications to Borsuk-Ulam and Bourgin-Yang theorems, Ergodic Theory Dynam. Systems, 8 (1988), Charles Conley Memorial Issue, 73-85.

[28] Flexner, W.; On topological manifolds. Annals of Mathematics, Second Series, Vol. 32 (1931), no. 2, 393-406.

[29] _ _ The poincaré duality theorem for topological manifolds. Annals of Mathematics, Second Series, Vol. 32 (1931), no. 3, 539-548.

[30] Godement, R.; Topologie algébrique et théorie des faisceaux. 3rd ed., Actualités scientifiques et industrielles, Vol. 1252, Hermann, Paris, 1973.

[31] Greenberg, M. and Harper, J.; Algebraic topology, a first course, 3rd ed., Mathematics Lecture Note Series, Benjamin/Cummings Publishing Company, New York, 1981.

[32] Grothendieck, A.; A general theory of fibre spaces with structure sheaf. University of Kansas (1955).

[33] _ _ Sur quelques points d'algèbre homologique. The Tohoku Mathematical Journal, Second Edition 9 (1957), 119-221. 
[34] Hara, Yasuhiro, The degree of equivariant maps. Topology and its Applications, 148 (2005), 113-121.

[35] Harlap, A.; Local homology and cohomology, homology dimension and generalized manifolds, Mathematics of the USSR-Sbornik, Vol. 25 (1975), no. 3, 323-349.

[36] Hu, S.; Homological algebra, Holden-day, Inc. (1968).

[37] Jakobsche, W.; $\mathbb{Z}_{p}$-cohomology manifold with no $\mathbb{Z}_{p}$-resolution. Fundamenta Mathematicae, Vol. 137 (1991), no. 3, 157-159.

[38] Jaworowski, Jan, The degree of maps of free $G$-manifolds and the average, Journal Fixed Point Theory appl.,1 (2007), 111-121.

[39] Karimov, U. and D. Repovš, D.; Examples of cohomology manifolds which are not homologically locally connected. Topology and its Applications, 155 (2008), 1169-1174.

[40] Kushkuley, A. and Balanov, Z.; A comparison principle and extension of equivariant maps. Manuscripa Math., Vol. 83 (1994), 239-264.

[41] Leray, J.; L'anneau d'homologie d'une represéntation. Comptes Rendus de l'Academémie des sciences 222 (1946), 1366-1368.

[42] _ _ Structure de l'anneau d'homologie d'une represéntation. Comptes Rendus de l'Academémie des sciences 222 (1946), 1419-1422.

[43] Lefschetz, S.; Topology. American Mathematical Society Colloquium Publications, Vol. 12 (1930).

[44]_and Flexner, W.; On the duality theorems for the Betti numbers of topological manifolds. Proceedings of the National Academy of Sciences of the United States of America, Vol. 16 (1930), no. 8, 530-533.

[45] _ _ On generalized manifolds. American Journal of Mathematics, Vol.55 (1933), no. 2, 469-504.

[46] _ ; On locally connected and related sets. Duke Mathematical Journal, Vol.2 (1936), 435-442. 
[47] Mitchell, W.; Homology manifolds, inverse systems and cohomological local connectedness. Journal of the London Mathematical Society, 19 (1979), no. $2,348-358$.

[48] Munkres, J.; Topology, Prentice Hall (2000).

[49] Nagata, Jun, Modern dimension theory, Sigma series in pure mathematics, Vol. 2, Heldermann Verlag, 1983.

[50] Oliver, Robert, A proof of the Conner conjecture, Annals of Mathematics 103 (1976), 637-644.

[51] Olum, P.; Mappings of manifolds and the notion of degree. Annals of Mathematics, Vol. 8 (1953), no. 53, 458-480.

[52] Pontrjagin, L.; Über den algebraischen inhal topologische dualitätssäze. Mathematische Annalen, Vol. 105 (1931), 165-205.

[53] Raymond, F.; Separation and union theorems for generalized manifolds with boundary, Michigan Mathematical Journal 7 (1960), 7-21.

[54] _ _ Local cohomology groups with closed support. Mathematische Zeitschrift, 76 (1961), 31-41.

[55] Sklyarenko, E.; Two orientations. Mathematical Notes, Vol. 83 (2008), 8896.

[56] _ ; The homological degree and hopf absolute degree. Sbornik Mathematics, Vol. 199 (2008), no. 11, 1687-1713.

[57] Smith, Paul, Transformations of finite period II, Annals of Mathematics, Second Series, 40, no. 3 (1939), 690-711.

[58] Tennison, B.; Sheaf theory. 2nd ed., London Mathematical Society Lecture Note Series, Vol. 20, Cambridge University Press, 1975.

[59] White, P.; On the union of two generalized manifolds. Annalin della Scuola Normale Superiore di Pisa, Classe di Scienze, Third Series, Vol. 4 (1950), no. 3-4, 231-243. 
[60] Wilder, R.; A converse of the Jordan-Brouwer separation theorem in three dimensions. Transactions of the American Mathematical Society, Vol 32 (1930), 632-657.

[61] _ ; Generalized closed manifolds in n-space. Annals of Mathematics, Second Series, Vol. 35 (1934), no. 4, 876-903.

[62] _ , Topology of manifolds, American Mathematical Society colloquium publications, American Mathematical Society, 1949. 


\section{Índice Remissivo}

Ações concordantes, 22

Aplicação órbita, 21

Aplicação equivariante, 21

Aplicação orientável, 18

Classe fundamental, 17

clc, 13

Cohomologia de feixes, 57

absoluta, 57

reduzida, 13

relativa, 61

Conjunto dos pontos fixos, 21

Dimensão

cohomológica, 10

topológica, 9

Dualidade de Poincaré, 17

Espaço órbita, 21

Família de suportes, 54

fechado de $X, 54$

compacto, 54

Feixe, 49 constante, 51

de homologia, 11

gerado por um prefeixe, 53

Imagem inversa, 50

localmente constante, 51

orientação, 11, 14

produto tensorial, 54

produto torção, 54

quociente, 53

restrição, 50

Grau de uma aplicação, 18

Grupo de transformações, 21

Grupo Weyl, 21

HCL, 8

hlc, 13

Homologia de Borel-Moore, 64

Homologia de feixes

relativa, 66

Homomorfismo

de feixes, 50

induzido 
cohomologia, 59

orientação, 22

prefeixes, 52

indice de Fadell-Husseini, 2, 25

Prefeixe, 51

constante, 52

de seções, 52

Seção, 50

com suporte, 55

Seqüência longa

feixes, 58, 65

par, 61

Subfeixe, 50

Subgrupo de isotropia, 21

Suporte, 55

Variedade

de homologia, 14

orientavel, 14

generalizada, 14

orientável, 14 